\title{
A Science Strategy fOR Space Physics
}

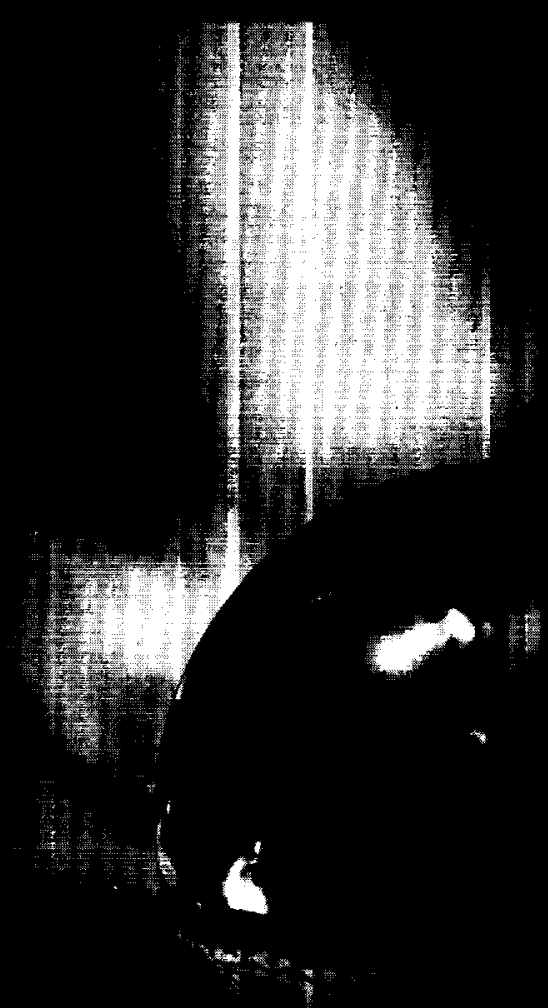

$$
\begin{aligned}
& A+A \\
& \text { N-B.-. } \\
& 0.3 .57
\end{aligned}
$$

(t)

\section{SPACE Studies BOARD}

\section{Matiomal Research Coumall}




\section{A Science Strategy for Space Physics}

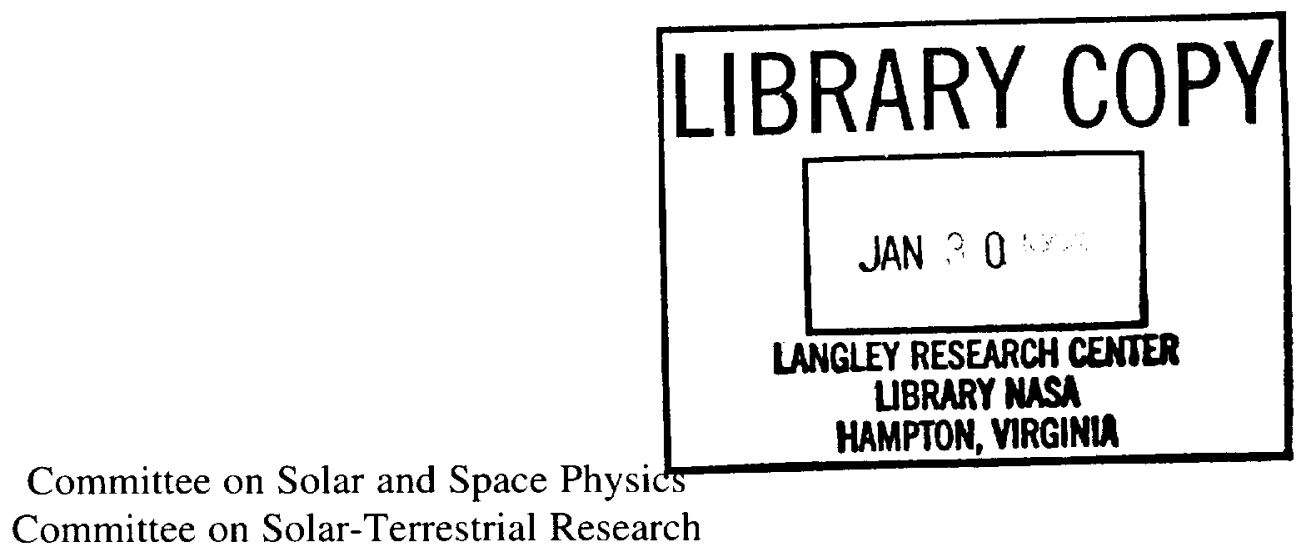

Space Studies Board

Commission on Physical Sciences, Mathematics, and Applications

Board on Atmospheric Sciences and Climate

Commission on Geosciences, Environment, and Resources

National Research Council

Washington, D.C. 1995 
NOTICE: The project that is the subject of this report was approved by the Governing Board of the National Research Council, whose members are drawn from the councils of the National Academy of Sciences, the National Academy of Engineering, and the Institute of Medicine. The members of the committees responsible for the repurt were chosen for their special competences and with regard for appropriate balance.

This report has been reviewed by a group other than the authors according to procedures approved by a Report Review Committee consisting of members of the National Academy of Sciences, the National Academy of Engineering, and the Institute of Medicine.

The National Academy of Sciences is a private, nonprofit, self-perpetuating society of distinguished scholars engaged in scientific and engineering research. dedicated to the furtherance of science and technology and to their use for the general welfare. Upon the authority of the charter granted to it by the Congress in 1863, the Academy has a mandate that requires it to advise the federal government on scientific and technical matters. Dr. Bruce M. Alberts is president of the National Academy of Sciences.

The National Academy of Engineering was established in 1964, under the charter of the National Academy of Sciences. as a parallel organization of outstanding engineers. It is autonomous in its administration and in the selection of its members, sharing with the National Academy of Sciences the responsibility for advising the federal government. The National Academy of Engineering also sponsors engineering programs aimed at meeting national needs, encourages education and research, and recognizes the superior achievements of engineers. Dr. Harold Liebowitz is president of the National Academy of Engineering.

The Institute of Medicine was established in 1970 by the National Academy of Sciences to secure the services of eminent members of appropriate professions in the examination of policy matters pertaining to the health of the public. The Institute acts under the responsibility given to the National Academy of Sciences by its congressional charter to be an adviser to the federal government and, upon its own initiative, to identify issues of medical care, research, and education. Dr. Kenneth $I$. Shine is president of the Institute of Medicine.

The National Research Council was organized by the National Academy of Sciences in 1916 to associate the broad community of science and technology with the Academy's purposes of furthering knowledge and advising the federal government. Functioning in accordance with general policies determined by the Academy, the Council has become the principal operating agency of both the National Academy of Sciences and the National Academy of Engineering in providing services to the government, the public, and the scientific and engineering communities. The Council is administered jointly by both Academies and the Institute of Medicine. Dr. Bruce M. Alberts and Dr. Harold Liebowitz are chairman and vice chairman, respectively, of the National Research Council.

Support for this project was provided by Research Grant NASW 4627 between the National Academy of Sciences and the National Aeronautics and Space Administration.

Cover: Collage images courtesy of Syun-lchi Akasofu, Eric Christian, Leon Golub, and Serge Koutchmy. Cover designed by Penny Margolskee.

Copies of this report are available from

Space Studies Board

National Research Council

2101 Constitution Avenue, N.W.

Washington, D.C. 20418

Copyright 1995 by the National Academy of Sciences. All rights reserved.

Printed in the United States of America 


\section{COMMITTEE ON SOLAR AND SPACE PHYSICS}

MARCIA NEUGEBAUER,* Jet Propulsion Laboratory, Chair

JANET G. LUHMANN, University of California, Berkeley, Successor Chair

SPIRO ANTIOCHOS, Naval Research Laboratory

JANET U. KOZYRA, University of Michigan

DONALD G. MITCHELL, Applied Physics Laboratory

JONATHAN F. ORMES, ${ }^{*}$ Goddard Space Flight Center

GEORGE K. PARKS,* University of Washington

DOUGLAS RABIN, ${ }^{*}$ National Optical Astronomy Observatory

ARTHUR D. RICHMOND, National Center for Atmospheric Research

HARLAN SPENCE, Boston University

MICHELLE F. THOMSEN, Los Alamos National Laboratory

ROGER K. ULRICH, University of California, Los Angeles

RONALD D. ZWICKL, ${ }^{*}$ National Oceanic and Atmospheric Administration

Staff

RICHARD C. HART, Executive Secretary

DAVID H. SMITH, Continuing Executive Secretary

CARMELA CHAMBERLAIN, Senior Program Assistant

ALTORIA B. ROSS, Continuing Senior Program Assistant

ERIN C. HATCH, Research Assistant

*Term expired in 1994. 
COMMITTEE ON SOLAR-TERRESTRIAL RESEARCH

MARVIN A. GELLER, State University of New York, Chair GUY P. BRASSEUR, National Center for Atmospheric Research CYNTHIA A. CATTELL, University of Minnesota JOHN V. EVANS, COMSAT Laboratories

PAUL A. EVENSON, University of Delaware JOSEPH F. FENNELL, The Aerospace Corporation JOHN T. GOSLING, Los Alamos National Laboratory

SHADIA RIFAI HABBAL, Harvard-Smithsonian Center for Astrophysics GORDON HURFORD, California Institute of Technology DAVID J. McCOMAS, ${ }^{*}$ Los Alamos National Laboratory JAMES F. VICKREY.* SRI International

*Term expired in 1994. 


\section{SPACE STUDIES BOARD}

CLAUDE R. CANIZARES, Massachusetts Institute of Technology, Chair

LOUIS J. LANZEROTTI,* AT\&T Bell Laboratories, Former Chair

JOHN A. ARMSTRONG, IBM Corporation (retired)

LAWRENCE BOGORAD, Harvard University

JOSEPH A. BURNS, ${ }^{* *}$ Comell University

JOHN J. DONEGAN, U.S. Navy (retired)

ANTHONY W. ENGLAND, University of Michigan

JAMES P. FERRIS, * Rensselaer Polytechnic Institute

DANIEL J. FINK, D.J. Fink Associates, Inc.

HERBERT FRIEDMAN, ${ }^{*}$ Naval Research Laboratory

MARTIN E. GLICKSMAN, Rensselaer Polytechnic Institute

RONALD GREELEY, Arizona State University

BILL GREEN, former member, U.S. House of Representatives

HAROLD J. GUY,** University of California, San Diego

NOEL W. HINNERS, Lockhced Martin Astronautics

ROBERT A. LAUDISE,* AT\&T Bell Laboratories

RICHARD S. LINDZEN, ${ }^{*}$ Massachusetts Institute of Technology

JANET G. LUHMANN, University of California, Berkeley

JOHN H. McELROY, University of Texas, Arlington

WILLIAM J. MERRELL, JR., ${ }^{*}$ Texas A\&M University

ROBERTA BALSTAD MILLER, Consortium for International Earth Sciences Information Network

BERRIEN MOORE III, University of New Hampshire

NORMAN F. NESS, * University of Delaware

MARCIA NEUGEBAUER,* Jet Propulsion Laboratory

MARY JANE OSBORN, University of Connecticut Health Center

SIMON OSTRACH, Case Westem Reserve University

JEREMIAH P. OSTRIKER, * Princeton University

CARLÉ M. PIETERS, Brown University

JUDITH PIPHER, ${ }^{* *}$ University of Rochester

MARCIA J. RIEKE, University of Arizona

ROLAND SCHMITT, Rensselaer Polytechnic Institute (retired)

JOHN A. SIMPSON, University of Chicago

WILLIAM A. SIRIGNANO,* University of Califomia, Irvine

JOHN W. TOWNSEND,* NASA (retired)

FRED W. TUREK, ${ }^{*}$ Northwestern University

ARTHUR B.C. WALKER, JR., ${ }^{* *}$ Stanford University

MARC S. ALLEN, Director

*'Term expired in 1994

**Term expired in 1995 . 
JOHN A. DUTTON, Pennsylvania State University, Chair

ERIC J. BARRON, Pennsylvania State University

WILLIAM L. CHAMEIDES, Georgia Institute of Technology

CRAIG E. DORMAN, Department of Defense

FRANCO EINAUDI, Goddard Space Flight Center

MARVIN A. GELLER, State University of New York, Stony Brook

PETER V. HOBBS, University of Washington

WITOLD F. KRAJEWSKI, University of Iowa

MARGARET A. LeMONE, National Center for Atmospheric Research

DOUGLAS K. LILLY, University of Oklahoma

RICHARD S. LINDZEN, Massachusetts Institute of Technology

GERALD R. NORTH, Texas A\&M University

EUGENE M. RASMUSSON, University of Maryland

ROBERT J. SERAFIN, National Center for Atmospheric Research

Staff

WILLIAM A. SPRIGG, Director

H. FRANK EDEN, Senior Program Officer

MARK D. HANDEL, Senior Program Officer

DAVID H. SLADE, Senior Program Officer

ELLEN F. RICE, Reports Officer

DORIS BOUADJEMI, Administrative Assistant

THERESA M. FISHER, Administrative Assistant

MARK BOEDO, Project Assistant 


\section{COMMISSION ON PHYSICAL SCIENCES, MATHEMATICS, AND APPLICATIONS}

ROBERT J. HERMANN, United Technologies Corporation, Chair STEPHEN L. ADLER, Institute for Advanced Study

PETER M. BANKS, Environmental Research Institute of Michigan SYLVIA T. CEYER, Massachusetts Institute of Technology

L. LOUIS HEGEDUS, W.R. Grace and Co.

JOHN E. HOPCROFT, Cornell University

RHONDA J. HUGHES, Bryn Mawr College

SHIRLEY A. JACKSON, U.S. Nuclear Regulatory Commission

KENNETH I. KELLERMANN, National Radio Astronomy Observatory

KEN KENNEDY, Rice University

HANS MARK, University of Texas, Austin

THOMAS A. PRINCE, California Institute of Technology

JEROME SACKS, National Institute for Statistical Sciences

L.E. SCRIVEN, University of Minnesota

LEON T. SILVER, California Institute of Technology

CHARLES P. SLICHTER, University of Illinois at Urbana-Champaign

ALVIN W. TRIVELPIECE, Oak Ridge National Laboratory

SHMUEL WINOGRAD, IBM T.J. Watson Research Center

CHARLES A. ZRAKET, MITRE Corporation (retired)

NORMAN METZGER, Executive Director 


\section{COMMISSION ON GEOSCIENCES, ENVIRONMENT, AND RESOURCES}

M. GORDON WOLMAN, Johns Hopkins University, Chair

PATRICK R. ATKINS, Aluminum Company of America

EDITH BROWN WEISS, Georgetown University Law Center

JAMES P. BRUCE, Canadian Climate Program Board

WILLIAM L. FISHER, University of Texas, Austin

EDWARD A. FRIEMAN, Scripps Institution of Oceanography, University of California, La Jolla GEORGE M. HORNBERGER, University of Virginia

W. BARCLAY KAMB, California Institute of Technology

PERRY L. McCARTY, Stanford University

S. GEORGE PHILANDER, Princeton University

RAYMOND A. PRICE, Queen's University of Kingston

THOMAS C. SCHELLING, University of Maryland

ELLEN SILBERGELD, University of Maryland Medical School

STEVEN M. STANLEY, Johns Hopkins University

VICTORIA J. TSCHINKEL, Landers and Parsons

Staff

STEPHEN RATTIEN, Executive Director

STEPHEN D. PARKER, Associate Executive Director

MORGAN GOPNIK, Assistant Executive Director

JAMES MALLORY, Administrative Officer

SANDRA FITZPATRICK, Administrative Associate 


\section{Contents}

Part I-Introduction to Space Physics

9

Part II-The Key Topics in Space Physics Research

1 Mechanisms of Solar Variability 23

2 The Physics of the Solar Wind and the Heliosphere 32

3 The Structure and Dynamics of Magnetospheres and Their Coupling to Adjacent Regions

4 The Middle and Upper Atmospheres and Their Coupling to Regions Above and Below 



\section{Summary}

This report by the Committee on Solar and Space Physics and the Committee on Solar-Terrestrial Research recommends the major directions for scientific research in space physics for the coming decade. As a field of science, space physics has passed through the stage of simply looking to see what is out beyond Earth's atmosphere. It has become a "hard" science, focusing on understanding the fundamental interactions between charged particles, electromagnetic fields, and gases in the natural laboratory consisting of the galaxy, the Sun, the heliosphere, and planetary magnetospheres, ionospheres, and upper atmospheres. The motivation for space physics research goes far beyond basic physics and intellectual curiosity, however, because long-term variations in the brightness of the Sun vitally affect the habitability of the Earth, while sudden rearrangements of magnetic fields above the solar surface can have profound effects on the delicate balance of the forces that shape our environment in space and on the human technology that is sensitive to that balance.

The several subfields of space physics share the following objectives:

- To understand the fundamental laws or processes of nature as they apply to space plasmas and rarefied gases both on the microscale and in the larger, complex systems that constitute the domain of space physics;

- To understand the links between changes in the Sun and the resulting effects at the Earth, with the eventual goal of predicting the significant effects on the terrestrial environment; and

- To continue the exploration and description of the plasmas and rarefied gases in the solar system.

Significant progress has been made in the more than three-decade history of space research. Many space plasma and rarefied gas phenomena have been characterized and are well understood, but many others are still under investigation and new discoveries continue to be made. Space physics asks fundamental questions about how plasmas are energized; about how the energy is redistributed with the result that a few particles are taken out of a near-thermal distribution and accelerated to superthermal or very high energies; about the specific roles played by magnetic fields in transferring energy from plasmas to particles and vice versa; about instabilities and interactions between waves and particles in a plasma; about the generation of magnetic fields through convection and rotation; and about the complex physical processes that operate in boundary layers between regions of different types of plasmas and rarefied gases. Some plasma configurations or particle distributions are known to be unstable and to relax spontaneously to a more stable state with the release of free energy, but there are many others for which the instabilities and wave-particle interactions are not yet understood. Determining the physics of such relaxation processes is fundamental to understanding and eventually being able to predict disturbances such as solar eruptions and geomagnetic storms, both of which can have important impacts on a technological society.

This strategy identifies five key scientific topics to be addressed in space physics research in the coming decade. For each of these topics, the report presents the scientific background and discusses why the topic 


\section{Box 1 \\ The Key Topics in Space Physics Research}

- Mechanisms of solar variability

- The physics of the solar wind and the heliosphere

- The structure and dynamics of magnetospheres and their coupling to adjacent regions

- The middle and upper atmospheres and their coupling to regions above and below

- Plasma processes that accelerate very energetic particles and control their propagation

is important, describes the current program for research on the topic, and then recommends, in priority order, research activities for the future. As is made clear in the main text, each of these five diverse topics is linked by a number of basic themes. Even though this strategy does not address specific proposals for future programs or missions, consideration is given to the practical aspects of carrying out the recommended investigations. The rationale for the research priorities is driven not only by scientific priority, but also by considerations such as current plans, near-term budget constraints, technological readiness, and balance between large- and smallscale endeavors. The five topics (Box 1), which are not prioritized, and the prioritized recommendations for research in each topic are briefly summarized as follows.

\section{THE KEY TOPICS IN SPACE PHYSICS RESEARCH}

\section{Mechanisms of Solar Variability}

The Sun is a variable star on time scales of milliseconds to centuries or more. These variations occur not only in visible light. but also at radio, ultraviolet, $x$-ray, and $\gamma$-ray wavelengths and in the emission of the solar wind and energetic particles. Although solar variability ultimately arises from the interaction of magnetic fields and differential rotation inside the Sun, the Sun's interior dynamics are largely unknown. The new tool of helioseismology is being used to probe the solar interior; it has shown that the Sun's rotation rate does not increase inward as had previously been postulated and thus rules out the "standard" model of the dynamo that generates the solar magnetic field. At least at the solar surface, and perhaps in the interior as well, the magnetic fields are confined to flux tubes-rope-like structures with diameters of about $100 \mathrm{~km}$ that are too small to be resolved from Earth. It has been suggested that the twisting and shearing of these flux tubes lead to bursts of high-speed solar wind, called coronal mass ejections, and to solar flares, but the trigger mechanisms for those violent events are not yet known.

On longer time scales, complacency about the simplicity of the Sun has been upset by the discovery and documentation in the historical record that the Sun has undergone periods of low activity. The association of the Maunder Minimum period ( 1645 to 1715), when few sunspots appeared on the solar surface for roughly 70 years, with the Little Ice Age, when Europe experienced exceptionally cold winters, has potentially serious implications for society should a similar episode occur under current conditions. In addition, confidence in current understanding of the solar interior was upset by the discordantly low flux of solar neutrinos observed in several experiments. It is not yet known whether this disagreement derives from errors in neutrino physics or from errors in understanding of the solar interior.

The research priorities for advancing the understanding of solar variability are as follows:

- Use helioseismology to study the structure and dynamics of the solar surface and interior over a full solar cycle, to obtain information on the interior changes that cause solar cycles.

- Assure continuity of total and spectral irradiance measurements, supported by spatially resolved spectrophotometry, to investigate correlations between solar magnetic activity and solar output variations and thereby to understand how they are coupled.

- Measure high-energy radiation and particles from flares and coronal mass ejections with good angular resolution, good spectral resolution, and wide spectral coverage to determine what drives each of those phenomena and how they contribute to the solar output at high energies. 
- Observe surface magnetic fields, velocities, and thermodynamic properties with enough spatial resolution $(<150 \mathrm{~km}$, with an ultimate goal of $<100 \mathrm{~km})$ to study small-scale structures such as flux tubes that may play a decisive role in solar activity and the generation of solar outputs.

- Make global-perspective measurements of the solar surface magnetic and velocity fields and solar oscillations to measure the three-dimensional structure and long-term evolution of active regions and to detect weak but coherent global oscillations.

- Measure active regions with angular resolution of $\sim 1$ arc sec and temporal resolution of $\sim 10$ min for a duration of $\sim 10$ days without nighttime gaps to determine the magnetohydrodynamic history of their emergence, development, and decay and the physical scenario behind it.

\section{The Physics of the Solar Wind and the Heliosphere}

Some of the energy transported from the solar interior goes into heating the Sun's outer atmosphere, called the corona, to over a million degrees by processes that are currently the target of intense study. The hot corona in turn becomes the source of the solar wind, but there are still major questions about how this occurs. Further observations and numerical simulations are required to determine the relative importance of magnetic reconnection. explosive jets, tiny active regions called bright points, hydromagnetic waves, and the topology of the magnetic fields in the corona in accelerating the quasi-stationary solar wind. There are additional questions about the acceleration of the nonstationary or transient solar wind arising from explosive events called coronal mass ejections. New observations of the variable elemental composition and ion charge states of the solar-wind plasma are providing valuable clues concerning the acceleration of both types of solar wind.

As the solar wind flows out through the solar system, it blows a big bubble, called the heliosphere, in the interstellar medium. Because of its very large scale $(\sim 100 \mathrm{AU})$, the heliosphere provides a unique laboratory for studying plasma processes in relative isolation from boundary effects; from heliospheric studies it is possible to learn much about instabilities in expanding plasmas, the interaction of colliding plasmas, the generation and evolution of plasma waves and magnetohydrodynamic turbulence, and the acceleration and propagation of energetic particles in turbulent fields. Within the decadal time frame considered by this report, it will be possible to measure the latitudinal variations of heliospheric particles and fields over the full range of solar activity and to test theories about the interaction of the solar wind with the interstellar gas and plasma.

The following priorities are identified for future research on the solar wind and the heliosphere:

- Continue to obtain and synthesize the data from the present constellation of heliospheric spacecraft and from the interplanetary cruise phases of planetary missions in order to characterize the global and solar-cycledependent properties of the heliosphere and its interactions with the interstellar medium.

- Carry out in situ observations of the solar corona to explore and characterize the region of acceleration of the solar wind and the physical processes responsible for that acceleration.

- Obtain new types of data required to reveal the mechanisms responsible for the transport of energy, including wave motions (periods of 1 to $10 \mathrm{~s}$ ), from the solar surface into the chromosphere and corona to understand how these are heated.

- Carry out stereo imaging of the solar corona to reveal the three-dimensional structure of coronal features without the ambiguity caused by integration along the line of sight.

- Develop and use techniques for the remote sensing of the coronal magnetic field in order to improve knowledge of the acceleration of the solar wind and of the initiation of coronal mass ejections.

- Make in situ measurements of the outer heliospheric boundaries and the interstellar medium with instruments specifically designed for those purposes.

\section{The Structure and Dynamics of Magnetospheres and Their Coupling to Adjacent Regions}

Some of the most visually awe-inspiring, yet poorly understood, terrestrial phenomena are a direct consequence of the interaction of the variable Sun and solar wind with the Earth. Auroral displays, usually confined to high latitudes, episodically descend into the temperate zones during periods of extreme solar activity. The aurora is only one manifestation of the complex chain of physical events and connections that link the energy output of the Sun with the Earth's magnetosphere, ionosphere, and atmosphere. 
As the solar wind reaches the Earth, some of it enters the magnetosphere via several different processes and paths and affects the circulation and dynamics of the plasma within the magnetosphere. The interplanetary magnetic field can become temporarily connected to the geomagnetic field. but the physics of the reconnection process is not yet well understood. Once within the magnetosphere, the energy from the solar wind cascades through the system and some is released catastrophically in events whose trigger mechanisms and extent are not well known. Flows of thermal and energetic plasma, large-scale current systems, magnetic perturbations, and imposed electric fields provide the basic links between the magnetosphere and the ionosphere. The ionosphere provides feedback to the magnetosphere in the form of ion outflow, conductivity changes, and dynamo fields. There is a continual reconfiguration of this system as the solar wind and its embedded magnetic field change in response to solar and interplanetary dynamics and energetics.

The past and current program, based primarily on in situ measurements, is providing an understanding of the magnetosphere that is strong in terms of local phenomena and a statistical picture of the global structure, but weak in terms of global dynamics. Researchers now know that most transport processes take place within narrow boundary layers connecting regions with very different plasma conditions. The frontier issues for the future center on the global magnetospheric dynamics in response to the solar wind driver, and the physical mechanisms that determine the coupling between regions. Many of the outstanding questions in magnetospheric physics will be addressed by global magnetospheric imaging, a new addition to the techniques available for magnetospheric research. In addition, studies of the magnetospheric environments of other planetary bodies can also yield important physical insights into the mechanisms that drive the dynamical behavior of the Earth's magnetosphere.

The following priorities are identified for future progress on this topic:

- Reap the full scientific potential of the International Solar-Terrestrial Physics program and its coordinated programs to advance understanding of the transport of mass, momentum, and energy throughout the solar wind. and the magnetosphere and ionosphere systems.

- Simultaneously image the plasma and energetic particle populations in the aurora, plasmasphere, ring current, and inner plasma sheet to study the global structure and large-scale interactions of magnetospheric and ionospheric regions during different levels of solar and geomagnetic activity.

- Maintain the full complement of particle and field instruments on current and future planetary missions to gain increased understanding of the formation and dynamics of diverse magnetospheres and ionospheres.

- Further develop and exploit ground-based facilities that image the ionospheric manifestations or "footpoints" of solar wind/magnetosphere coupling processes to complement the magnetospheric imaging initiative aimed at studying the global properties of the magnetosphere.

- Explore Mercury's magnetosphere to understand the role of an ionosphere in coupling between the solar wind and planetary magnetospheres.

- Target localized regions that require greater understanding of the small-scale physical processes occurring there with high-resolution, multispacecraft measurements that take advantage of new smaller, lighter, more capable instruments and more sophisticated data-compression schemes.

\section{The Middle and Upper Atmospheres and Their Coupling to Regions Above and Below}

A complex interface exists between Earth's space environment and the lower atmosphere or troposphere where weather and climate occur. This interface includes the highly variable middle and upper regions of the atmosphere extending upward from a lower boundary at 10 to $15 \mathrm{~km}$ altitude. The middle and upper atmospheres have considerable practical as well as intellectual interest because most ozone resides there and because disturbances in the upper atmosphere and ionosphere caused by solar wind and magnetospheric variations can disrupt technological systems through their effects on satellite drag, communications, and induced ground currents.

The middle and upper atmospheres are strongly influenced by inputs of mass, momentum, and energy from both above and below. The absorption of variable solar ultraviolet and $\mathrm{x}$-radiation and of energetic particles not only heats the atmosphere, but also initiates chains of photochemical reactions and ionizes the upper atmosphere to form the ionosphere. Highly variable electric fields and currents originating above and below the upper atmosphere are major sources of energy and momentum to that region. Gravity, planetary, and tidal waves that originate partly from the lower atmosphere grow in amplitude as they propagate upward, where they contribute to the momentum and energy budgets of the middle and upper atmospheres and produce turbulence that influences 
mixing processes. There are major deficiencies in our knowledge of many of these inputs to the middle and upper atmospheres as well as of the multiple interactions and feedbacks that occur there.

The following priorities are identified for future research aimed at understanding this important interface between Earth's lower atmosphere and space:

- Exploit the exciting new capabilities of UARS, ${ }^{1}$ FAST, and CEDAR to provide the foundation for future advances in our understanding of the middle and upper atmospheres.

- Investigate the reaction of the middle and upper atmospheres to upward propagating waves from the lower atmosphere and energy inputs from space so that the sources of important features such as the quasi-biennial and semiannual oscillations and the causes of mesosphere/lower-thermosphere structure and variability can be understood.

- Study the long-term variations in the middle and upper atmospheres using a combination of consistent long-term satellite and ground-based measurements together with three-dimensional radiative-chemical-dynamical modeling to understand natural and anthropogenic changes in these regions.

- Develop methods to observe the time-dependent electrodynamics operating on microscales to global scales, both in the upper atmosphere-ionosphere-magnetosphere coupling regions so that feedback processes can be characterized, and in the regions above thunderstorms so that the effects of electrified clouds on the "global circuit" and on middle atmosphere chemistry and energetics can be characterized.

- Take advantage of opportunities to include carefully chosen, appropriate instruments on planetary orbiter missions to make measurements critical to understanding planetary aeronomy and its relation to terrestrial aeronomic processes.

\section{Plasma Processes That Accelerate Very Energetic Particles and Control Their Propagation}

Many of the plasma processes responsible for phenomena within the heliosphere probably also play a role in determining the properties of galactic cosmic rays, which are the only available sample of matter from outside the local solar neighborhood. Mass, charge, and energy spectrometers on existing and planned spacecraft can make in situ measurements of energetic particles throughout the heliosphere to study particle acceleration, fractionation, and transport in a variety of space plasma environments. Theories of acceleration mechanisms in larger-scale galactic structures such as supernova remnants make specific predictions about compositional changes that should take place at the highest energies attainable in those objects, but the theories have not yet been tested observationally.

Cosmic rays are confined to the galaxy by turbulent magnetic fields. Measurements of radioactive "clock" nuclei can be used to distinguish diffusive trapping in the galactic halo from the simpler phenomenological models used for many years. Gamma ray measurements can be used to trace the radioactive parent elements of positrons that should be accelerated by the shocks presumed to be the source of the galactic cosmic ray nuclei. Although fluxes of antiprotons and positrons produced in collisions of cosmic rays with gas in the interstellar medium can be calculated with some precision, a full understanding of the sources of antimatter in the cosmic radiation requires a new generation of measurements.

Nuclides heavier than nickel are produced by accretion of neutrons either in supernova explosions or during certain other phases of stellar evolution. Knowledge of the abundance of different cosmic ray elements and isotopes will allow the use of nucleosynthesis models to determine quantitatively the fraction of cosmic rays synthesized in each type of source. The abundance of actinide elements can be used as a radioactive clock to determine the time delay between the synthesis of these elements and their acceleration to cosmic ray energies.

The measurements necessary to address scientific issues concerning particle acceleration and propagation are as follows, in priority order:

- Complete the observations from the current and planned network of interplanetary spacecraft to study particle acceleration, fractionation, and transport.

- Extend direct composition measurements to $10^{15} \mathrm{eV}$ to probe the limits of acceleration and trapping mechanisms. 
- Measure abundances of radioactive isotopes above $1 \mathrm{GeV} /$ nucleon to search for evidence of an extended galactic magnetosphere and wind.

- Measure the spectra of positrons (10 MeV to $100 \mathrm{GeV})$ and antiprotons (100 MeV to $100 \mathrm{GeV})$ to determine where those particles are created and how they are accelerated.

- Measure isotope abundances for nuclei heavier than nickel and elemental abundances through the actinides to probe the plasma regions where the nuclei are synthesized and to measure the time scales involved.

\section{RECOMMENDED RESEARCH EMPHASES}

The specific programs required to obtain answers to the questions raised under each of the five key topics outlined above are quite different. However, they are united by four common elements or themes that the CSSP and CSTR consider to be the most important research emphases for space physics in the next decade.

\section{Complete currently approved programs.}

The space physics community must reap the benefits of the existing approved programs. A stable program permits the most efficient management and execution of high-priority research. In addition to the obvious scientific return, these ongoing programs provide the basis for developing future research directions. Space physicists will gain the maximum benefit from ongoing and approved missions by:

- Completing the diverse components of the International Solar-Terrestrial Physics program;

- Enhancing data analysis and interpretation efforts;

- Streamlining mission operations for all space physics missions;

- Carrying out extended missions for the uniquely placed Voyager (to the greatest possible heliocentric distance) and Ulysses (through the solar polar passes at solar maximum);

- Supporting essential observational programs that require long-duration databases; and

- Enhancing the effectiveness of some of the longer-duration programs by soliciting new ideas and analysis techniques from guest investigators and by ensuring easy access to archived data resulting from the various programs for use in "small science" research tasks.

\section{Exploit existing technologies and opportunities to obtain new results in a cost-effective manner.}

Much technology is already in place to take the next observational steps required to address many of the important questions in space physics outlined here. These steps include:

- Adapting existing instrumentation to the new generation of smaller spacecraft and more focused space missions;

- Placing space physics instruments on planetary, Department of Defense, and other spacecraft of opportunity;

- Utilizing suborbital platforms such as rockets and long-duration balloons for both science objectives and instrument development; and

- Supporting, where appropriate, activities at unique ground sites such as in the polar cap.

3. Develop the new technology required to advance the frontiers of space physics.

In order to achieve several high-priority objectives, or to lower the cost of projects, the limits of technology must be pushed in the following ways:

- Developing methods to approach the Sun ever more closely to open one of the most exciting new frontiers of space science;

- Producing new spacecraft and instruments based on lightweight structure and miniature electronics;

- Designing a new generation of instrumentation for remote global imaging of magnetospheric, ionospheric, and solar wind plasmas; 
- Extending capabilities in suborbital techniques for both experimentation and instrument development;

- Exploiting infrared instrumentation for solar physics; and

- Devising techniques to explore the region between the altitudes reached by balloons and those reached by spacecraft.

\section{Support strongly the theory and modeling activities vital to space physics.}

Special emphasis should be given to the following topics:

- Recognizing that synergy between observations, modeling, and theory provides the optimum way of addressing the principal questions in space physics;

- Making numerical simulations of space physics systems more realistic by extending them to three dimensions, longer time durations, and a greater range of scale sizes, and by incorporating additional physical and chemical processes;

- Ensuring access to state-of-the-art computational facilities;

- Exploiting new insights gained from theory, especially the theory of nonlinear processes; and

- Revisiting earlier efforts to predict solar activity, such as coronal mass ejections and flares, using simulations combined with solar observations.

\section{NOTE}

1. A glossary of acronyms is included as the appendix, and the principal programs identified by the acronyms are described in the main text. 



\section{Part I}

\section{Introduction to Space Physics}

Space physics is the study of everything in the solar system above the lower atmospheres of the Earth and other solid bodies. For the purposes of this report, the entire Sun is included in the domain of space physics, together with the upper atmospheres, ionospheres, and magnetospheres of the planets, all the space between the planets, and the cosmic rays that enter the solar system from the galaxy. The purposes of this report are to summarize the state of space physics research in 1995, to identify and explain what the Committee on Solar and Space Physics and the Committee on Solar-Terrestrial Research believe are the most important topics to be addressed during the coming decade, and to suggest and prioritize the types of research efforts that might be most successful in answering the questions raised under each of those topics. 



\section{A BRIEF TOUR OF BASIC PHENOMENA}

After 35 years of measurements in space, and even longer periods of studying the Sun, cosmic rays, and the ionosphere from the ground or from balloons, space physicists have a reasonably complete description of the contents of the realm of space physics:

- The Sun is typical of stars of its mass and age, with its energy generated deep within by the conversion of light elements to heavier elements in a nuclear furnace. The detection of neutrinos produced by this conversion confirmed a fundamental cornerstone of modern astrophysics. The new tool of helioseismology allows us to deduce the distribution of matter, flows, and temperature below the visible surface of the Sun. The vast amount of energy that is constantly working its way from the nuclear furnace to the solar surface causes the gas in the outer layers of the Sun to churn and tumble over itself, which leads to the generation of complex magnetic fields of enormous strength. The combination of the magnetic fields and the churning gas is responsible for the activity seen on the solar surface, of which the best-known features are sunspots and flares.

- The Sun is constantly changing. No hour goes by without a rise or fall in its output of $x$-rays; rarely does a day go by without a solar flare; and no decade goes by without a peak and a valley in the Sun's brightness. Although the current decadal change in brightness is between $0.1 \%$ and $0.2 \%$, a sample of stars estimated to be similir to the Sun shows variability as large as $0.2 \%$ to $0.5 \%$. A comparison of the Sun's present level of activity with a sample of solar-type stars suggests that the Sun's total irradiance may have increased by about $0.25 \%$ since the period of low activity in the 17 th century known as the Maunder Minimum.

- It is this solar luminosity (or brightness) that heats the Earth and its atmosphere to habitable levels. Many researchers believe that variations in the luminosity of the Sun were responsible for changes in the climate of the northern temperate zone through the warm and cold centuries recorded by history. Two examples: First, the extremely high solar activity in the 13th century correlates well with abnormal warming that had catistrophic results for the semiarid southwestern United States. Second, during the past century there has been a gradual rise in the level of solar activity. It has been speculated that this rise has been accompanied by an increase in total solar output, and a rise in global temperature.

- Above its visible surface, the Sun has a tenuous atmosphere, called the corona. Figure 1 is a picture of the corona seen during eclipse, superimposed on a simultaneous $x$-ray image showing the distribution of hot, dense gases over the surface of the Sun and in the lower corona. The corona is superheated to over a million degrees. It is so hot that it is nearly completely ionized, existing in the fourth state of matter known as a plasma. At some places in the corona the magnetic field is organized into closed loops or bottles that confine the plasma, whereas at other places the field stretches out into space, allowing the outflow of plasma to form the solar wind. At times the magnetic configuration changes suddenly to release additional plasma into the wind.

- All the planets are embedded in the solar wind. When the solar wind encounters a planetary magnetic field that extends into space beyond the planet's atmosphere, a magnetosphere is created, because the sunward side of the planetary field is pushed back and confined by the pressure of the wind and, in the down-wind direction, the magnetic field is stretched into a long tail. A magnetosphere absorbs varying amounts of mass, momentum, and energy from the solar wind to drive a variety of phenomena that cause disturbances to a planet's magnetic field, including auroral displays. On Earth, such intense magnetic storms can induce destructive electromotive forces in power grids, disrupt communications, and lead to radiation damage of Earth satellites. When the solar wind encounters the atmosphere of a planetary body that has a weak magnetic field, the interaction may be quite different; at comets, for example, the interaction is dominated by the pickup of ionized cometary material by the solar wind over great distances, whereas at Venus with its higher gravitational field, the major part of the solar wind interaction is with the planet's ionosphere.

- Above the first $10 \mathrm{~km}$ of the Earth's atmosphere lie the layers that constitute the interface between the habitable regions of Earth and space. The ozone and other molecules in those layers absorb most of the harmful components of sunlight and energetic particles precipitating from the magnetosphere. The atmosphere expands and contracts in response to variations in ultraviolet light and x-rays from the Sun, thus dictating the necessary heights of satellite orbits, and occasionally accelerating reentry. The state of the atmosphere above $10 \mathrm{~km}$ is sensitive to variations in the input of ultraviolet and $x$-radiation from the Sun, the flux of energetic particles from the Sun and magnetosphere, and the fluxes from the lower atmosphere of greenhouse gases (principally carbon 


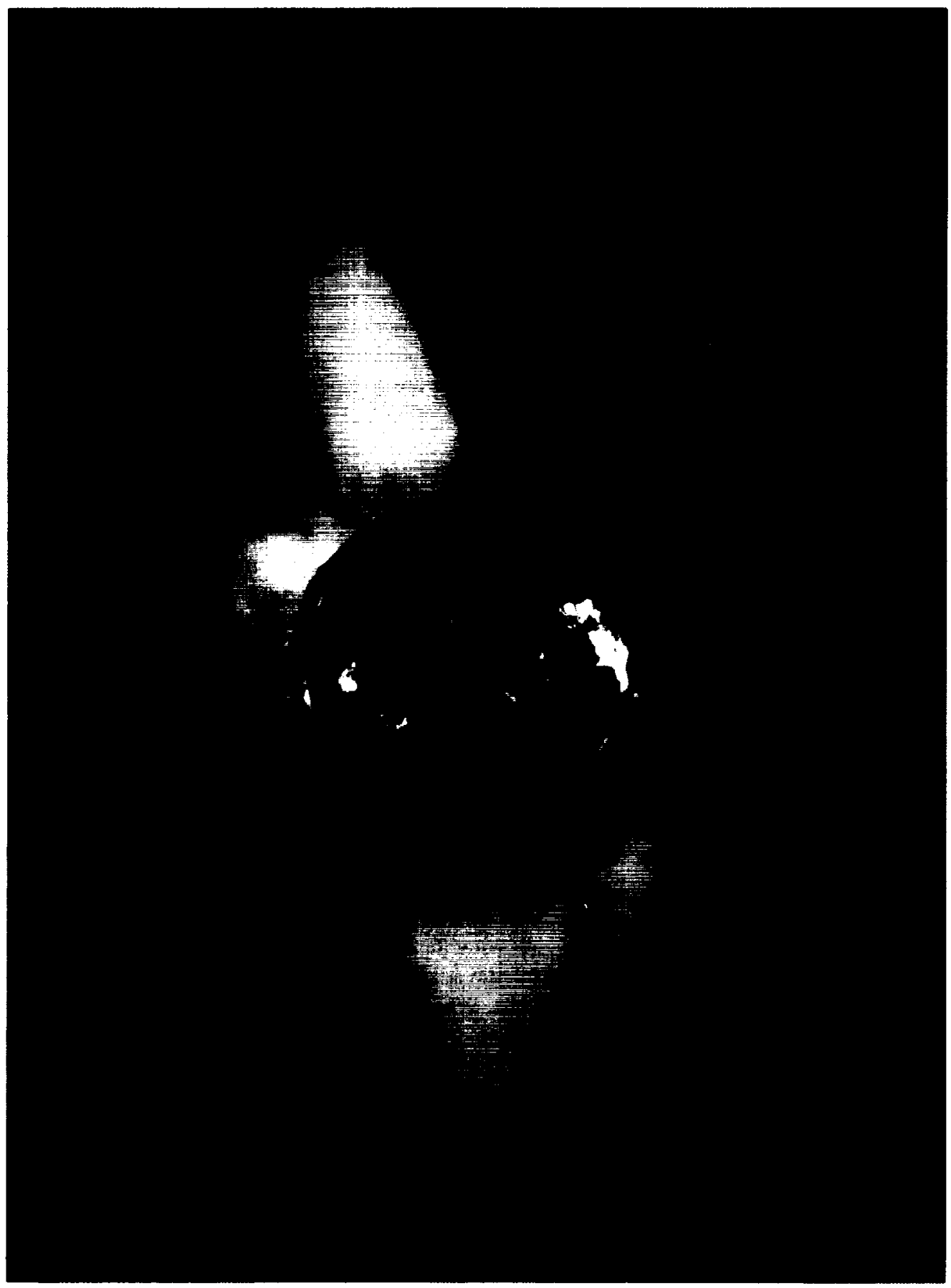

FIGURE I Sun and corona: A composite image of the Sun, with north at the top. The $x$-ray picture of the disk and inner corona was taken with Leon Golub's Normal Incidence X-ray Telescope by a rocket launched from White Sands, New Mexico. The white-light image of the outer corona was obtained simultaneously at 17:30 UT, July 11, 1991, by Serge Koutchmy when the Sun was in eclipse in Hawaii. The black ring visible in some places outside the $x$-ray solar disk is the moon as seen from Hawaii at the time of the eclipse. (Courtesy of Leon Golub, Smithsonian Astrophysical Observatory.) 
dioxide and methane) as well as gases that affect ozone chemistry (such as the chlorofluorocarbons and methyl bromide).

- The solar wind continues well past the outermost planet and is expected to come to a stop only when it reaches a pressure balance with the interstellar medium; the region of space occupied by the solar wind is called the heliosphere. Cosmic rays, which are nuclear particles accelerated to enormous energies elsewhere in the galaxy, push their way into the heliosphere in opposition to the outwardly expanding solar wind. The sources of cosmic rays are apparently distributed throughout the disk of the Milky Way galaxy, and the particles fill the galaxy, perhaps driving a galactic wind in a manner analogous to that by which the Sun drives the solar wind. The study of their energy spectrum and composition permits inferences not only about the origin and evolution of the elements, but also about the structure and evolution of the galaxy.

- Space physicists now have some idea of the average properties-such as the density, temperature, flow patterns and speeds, and magnetic fields - at most locations within the heliosphere. The exceptions include the unexplored magnetosphere of Pluto (if it has one), some regions of other planetary magnetospheres, the innermost heliosphere, high solar latitudes under conditions of maximum solar activity, the distant outer region of the heliosphere, and the interstellar medium beyond the heliosphere.

This brief tour highlights some of the basic phenomena of space physics. Although there is great diversity in the settings and the details, only a few basic processes underlie much of the physics. Mass motions in ionized media lead to the creation of magnetic flux in natural dynamos, such as the solar interior and the Earth's upper atmosphere. Further mass motion and the interaction of charged particles with the field and with neutral gases lead to the dissipation of the magnetic energy through magnetic reconnection and other processes. Some of the energy is used to accelerate a small fraction of the matter to extraordinary energies.

For the most part, the emphasis in space physics research has shifted from exploration to trying to understand why the properties are as we observe them to be. Instead of asking only, What's out there?, space physicists are increasingly asking, How does it work? and How will it evolve? This is especially true of the dynamic events, from solar eruptions to terrestrial magnetic storms to environmental changes.

There is much still to be learned: Why is the output of neutrinos by the solar furnace so much less than the theoretically predicted value? How does the Sun generate the strong magnetic fields observed at its surface? What processes cause the solar variability observed on time scales from seconds to centuries? How is the solar corona heated, and how is the solar wind accelerated to the high speeds observed? How do changes in the solar wind affect the various planetary magnetospheres? How are processes in the Earth's magnetosphere, ionosphere, and different levels of the atmosphere coupled together? What are the processes responsible for the observed dynamical and chemical variability and for the energy budget of the different layers of the upper atmosphere? How do magnetic fields and plasma particles interact on the large scale of the heliosphere? What is the origin of cosmic rays, and how do they interact on galactic scales?

\section{THE OBJECTIVES OF SPACE PHYSICS}

The scientific objectives of space physics research are as follows:

- To understand the fundamental laws or processes of nature as they apply to space plasmas and rarefied gases both on the microscale and in the larger, complex systems that constitute the domain of space physics;

- To understand the links between changes in the Sun and the resulting effects at the Earth, with the eventual goal of predicting the significant effects on the terrestrial environment; and

- To continue the exploration and description of the plasmas and rarefied gases in the solar system.

\section{Fundamental Processes}

Plasmas are the fourth state of matter. A plasma behaves differently than does a solid, liquid, or gas because each of its charged particles reacts to the electric and magnetic fields generated by the locations and motions of all the other charged particles within a very large volume; in turn, the resulting changes in each particle's location or motion cause changes in the fields. After more than three decades of research, many plasma phenomena are well understood. One example is the propagation of a host of different types of waves (e.g., electromagnetic, electro- 
static, fast-and slow-mode magnetosonic. Alfvén, and various hybrid waves), at least at small amplitudes where nonlinear effects are not important. But many other plasma processes require further investigation, both observationally and theoretically. Some of the major processes of current concern in space physics are the following:

- Bulk energization or acceleration of plasmas;

- Acceleration of a few particles out of a near-thermal distribution to superthermal or very high energies;

- Magnetic reconnection in which the topology of the magnetic field is changed. sometimes very rapidly, and magnetic energy is converted to kinetic energy of the particles in the plasma:

- Instabilities and interactions between waves and particles in a plasma. Some plasma configurations or particle distributions are known to be unstable and to relax spontaneously to a state of lower free energy, but there are many others for which the details of the instabilities and wave-particle interactions are not yet understood:

- Wave-wave interactions, both in plasmas and neutral gases:

- Magnetohydrodynamic turbulence and turbulent diffusion: and

- Dynamo generation of magnetic fields in a star with a convective envelope (such as the Sun's).

It is seldom possible to study any single physical process in isolation from myriad other simultaneously occurring processes. In space, it is nature rather than the investigator that determines the observational parameters and the boundary conditions. Thus we are constrained to observe processes as they are found within large-scale systems, such as the Earth's magnetosphere. ionosphere. or mesosphere, the solar corona, or the heliosphere, to name only a few examples. But those large-scale systems are also of intrinsic interest in themselves. Consider the magnetosphere as one example. It continuously intersects flows of mass, momentum, and energy in the solar wind, absorbs a varying fraction of those flows, rearranges itself as it temporarily stores this mass and energy, and then sporadically sheds this input to maintain a steady state when averaged over the long term. Researchers still have much to learn about how the magnetosphere does all that using basic physical processes such as magneticfield reconnection, plasma instabilities. and a host of others.

\section{Application to the Terrestrial Environment}

The fundamental physics of rarefied gases and plasmas has practical application to the problem of understanding the impact of solar processes on the terrestrial environment. A recent NRC study' chaired by Judith Lean reached the following conclusions concerning solar influences on global change:

- Solar variations directly modify global surface temperature.

- Solar variations modify ozone and the structure of the middle atmosphere.

- The effects of solar variability in the Earth's upper atmosphere may possibly couple to the biosphere.

- It is not known whether or not other types of variability in the Earth s near-space environment couple to the biosphere.

- Improved knowledge of the Sun is required to understand and predict the influences of solar variability on global change.

The CSSP-CSTR endorses the prioritized recommendations for monitoring and scientific research developed by the Lean Committee: a summary of those recommendations is reproduced in Box 2 .

Disturbances propagating through the Earth's space environment are known to affect a number of modern technological systems. A schematic illustration of some of these effects, both in space and on the surface of the Earth, is shown in Figure 2. For example, the x-rays emitted during solar flares travel to Earth and are absorbed in the upper atmosphere. This leads to changes in the electron density and the currents in the lower ionosphere. which cause radio waves to propagate in different and often unexpected directions. Signals from communication satellites are similarly affected by severe geomagnetic activity. The variability of the Sun's radiation produces variable heating of the upper atmosphere, which results in increased satellite drag, and perhaps premature reentry.

Another impact on human systems comes in the form of energetic particles. The particle radiation level in the Earth's space environment can become hazardous for astronauts and electronic systems on satellites, as illustrated in Figure 2. The increased radiation due to solar energetic particle events and high levels of geomagnetic activity 


\section{Box 2 \\ Recommendations of the Report Solar Influences on Global Change}

The highest priority and most urgent activity for determining solar influences on global change is to:

1. Monitor the total and spectral solar irradiance from an uninterrupted, overlapping series of spacecraft radiometers employing in-flight sensitivity tracking.

So that the long term value of present solar monitoring is not lost, adequate temporal overlap to permit cross-calibration with future observations is critical. This goal must be achieved in an era of decreasing access to space.

In addition, the following activities will be needed to properly monitor, understand, and predict solar influences on global change. Pursuit of recommendations 2 to 6 is essential to the interdisciplinary research effort needed to provide an adequate scientific basis for global change policymaking. The actions of recommendations 7 to 12 are essential to ensure a complete understanding of all potential coupling mechanisms.

2. Conduct exploratory modeling and observational studies to understand climate sensitivity to solar forcing.

3. Understand and characterize, through analysis of solar images and surrogates, the sources of solar spectral (and hence total) irradiance variability.

4. Monitor, without interruption, the cycles exhibited by Sun-like stars and understand the implications of these observations for long term solar variability.

5. Monitor globally, over many solar cycles the middle atmosphere's structure, dynamics, and composition, especially ozone and temperature.

6. Understand the radiative, chemical, and dynamical pathways that couple the middle atmosphere to the biosphere, as well as the middle atmosphere processes that affect these pathways.

7. Monitor continuously, with improved accuracy and long term precision, the ultraviolet radiation reaching the Earth's surface.

8. Understand convection, turbulence, oscillations, and magnetic field evolution in the solar plasma so as to develop techniques for assessing solar activity levels in the past and to predict them in the future.

9. Monitor continuously the energetic particle inputs to the Earth's atmosphere.

10. Monitor the solar extreme ultraviolet spectral irradiance (at wavelengths less than $120 \mathrm{~nm}$ ) for sufficiently long periods to fully assess the long term variations.

11. Monitor globally over long periods the basic structure of the lower thermosphere and upper mesosphere so as to properly define the present structure and its response to solar forcing.

12. Conduct observational and modeling studies to understand the chemical, dynamical, radiative and electrical coupling of the upper atmosphere to the middle and lower atmospheres.

Note: Reprinted from pp. 10-12 of Board on Global Change. Solar Influences on Global Change, National Academy Press, Washington, D.C.. 1994. 


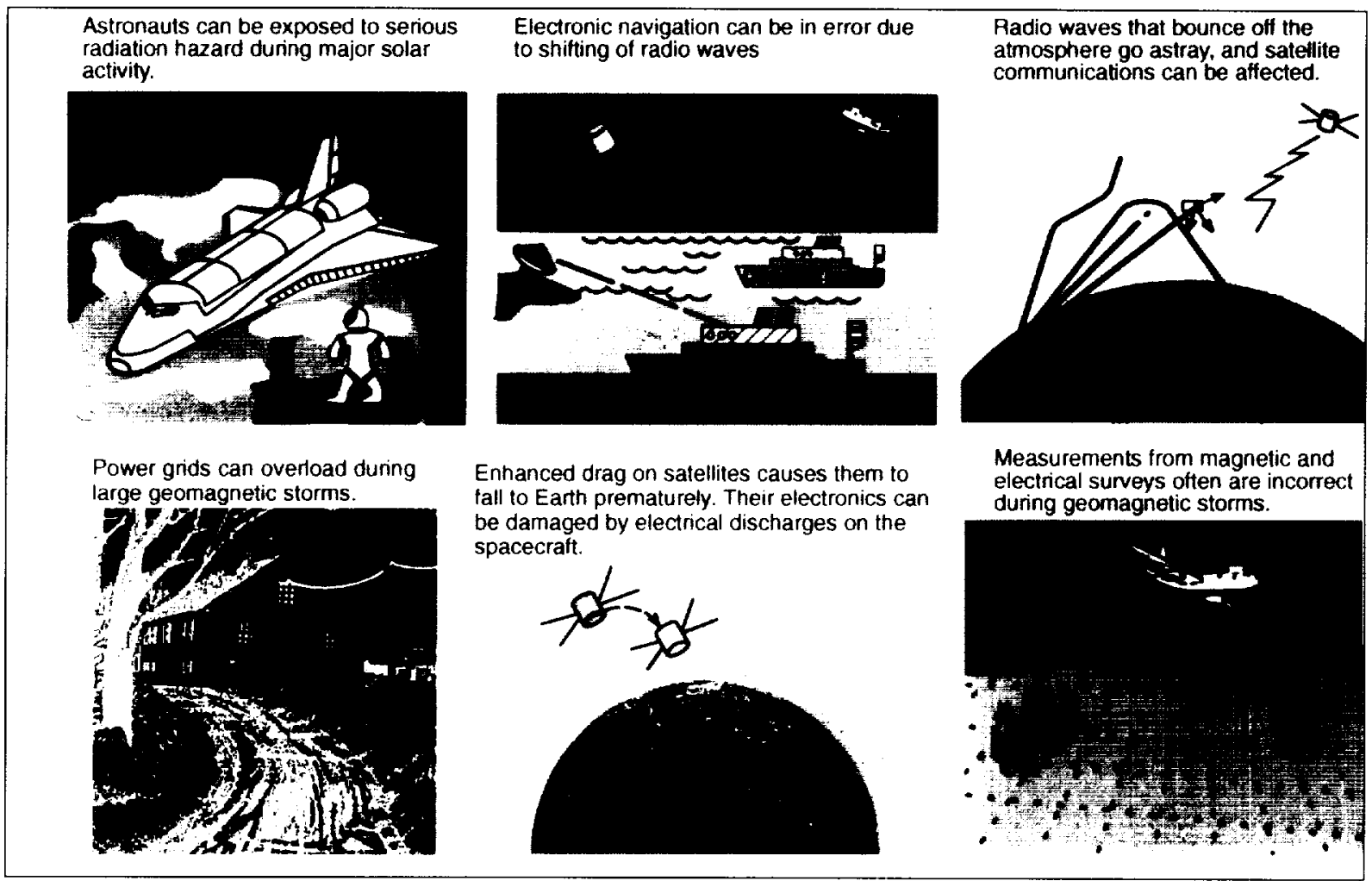

FIGURE 2 Some effects of solar-terrestrial disturbances. (Courtesy of the Space Environment Laboratory, NOAA.)

cause surface charging and deep dielectric charging on satellites, which can result in electronic faults and permanent damage to electrical systems. In the most extreme cases, satellites have become useless or inoperable due to these effects. High levels of geomagnetic activity are capable of disabling entire utility systems. Large-scale blackouts can have serious economic impacts even if power is restored in a few hours. The economic impact of a four-hour major blackout in France was estimated to be $\$ 1$ billion, while a somewhat longer major blackout of the Northeast section of the United States could result in a $\$ 3$ billion to $\$ 6$ billion loss. ${ }^{2}$ These problems are a consequence of geomagnetically induced currents that flow in the neutrals of grounded power transformers and lead to saturation of the core, with resulting transformer heating and failure. While there are numerous other examples, a clear trend has emerged: as technology advances, an increasing number of systems are affected by the highly variable space weather. ${ }^{3}$

\section{Exploration}

There are several important regions of the solar system where the properties of plasmas and rarefied gases have not yet been determined. Surprises almost certainly are in store for us when we obtain data from the first spacecraft sent to many of those regions. A recent example is the discovery of intense plasma waves, very high fluxes of energetic particles, and large fluxes of negative ions detected at comets. The lessons learned about fundamental physical processes, such as ion pickup, from the comet flybys of 1985 to 1992 have broad applicability in other large-scale systems, such as the outer heliosphere. Examples of regions about which space physicists currently know very little and that thus still need to be explored include the magnetic field in the local interstellar medium or the nature of the particle distributions or the wave field in the region close to the Sun where the solar wind is accelerated. 


\section{THE ROLE OF NEW TECHNOLOGY}

Advances in each of the three areas discussed above are enabled by the development and application of relevant new technology. Some progress can be made by sending old instruments to new places. However, many critical measurements described in this report require greater sensitivity or better resolution (be it spatial, temporal. energy, angular distribution, and so on) than can be achieved with currently available hardware. Advanced computers and software are required to generate models that can account for and visualize all the relevant spatial and temporal scales involved in some of the more challenging problems. Several of the high-priority objectives of space physics require a new generation of very lightweight, low-power instruments.

\section{BACKGROUND AND SCOPE OF THIS STUDY}

For many years, the Space Studies Board (SSB) and its predecessor, the Space Science Board, have provided scientific advice to NASA via the mechanism of discipline-specific strategy reports. Such reports are intended to assist NASA in developing the best possible scientific research programs for the future. In 1980, the Committee on Solar and Space Physics (CSSP) produced its first strategy report, Solar-System Space Physics in the 1980's: A Research Strategy, ${ }^{4}$ which is often referred to as the Kennel report. That report identified the scientific objectives for solar and space physics and described the program, experiments, and instruments required to continue progress on a broad front toward achieving those objectives. In 1985, the CSSP published An Implementation Plan for Priorities in Solar-System Space Physics ${ }^{5}$ (the Krimigis report), which, starting with the science objectives given in the Kennel report, developed a plan for addressing those objectives.

The Committee on Solar-Terrestrial Research (CSTR) of the Board on Atmospheric Sciences and Climate also published reports during the 1980s-Solar-Terrestrial Research for the 1980 's, ${ }^{6}$ and National Solar-Terrestrial Research Program ${ }^{7}$ - that recommended science programs in solar and space physics for all federal agencies involved in such research, including ground-based as well as space-based activities. In addition, during the decade of the 1980s a number of other reports were produced that addressed specific issues or areas of solar and space physics, including upper atmospheric research, the Middle Atmosphere program, international cooperation, the Explorer program, data management, ground-based solar physics, and long-term solar-terrestrial observations.

In 1992, the CSSP and CSTR, operating together as a single, federated committee, completed an assessment of the status of the field and a review of the federal agencies' responses to past recommendations (Assessment of Programs in Solar and Space Physics-1991). ${ }^{8}$ The CSSP-CSTR followed this assessment report with the report A Space Physics Paradox: Why Has Increased Funding Been Accompanied by Decreased Effectiveness in the Conduct of Space Physics Research?.9 The concluding recommendation of the "Paradox report" was that, in anticipation of an era of limited resources, the space physics community could ensure the most effective use of funding for space physics research by establishing priorities across the full spectrum of its scientific interests, encompassing both large-scale and small-scale activities. Other motivations for updating the science strategy for solar and space physics are the following:

- There have been both scientific and technological advances in the field in the 15 years since the publication of the Kennel report and the 10 years since the publication of the Krimigis report. Many of NASA's and NSF's plans for future programs address new scientific issues that were not considered in those earlier reports.

- Changes in the NASA organization have resulted in upper atmosphere research and cosmic-ray physics being administered as part of space physics; a broader scientific rationale and view are therefore required for the assessment and direction of NASA's space physics program.

- Changes in the roles of the shuttle and the space station in NASA's program, coupled with changes in the fiscal environment in which the relevant federal agencies operate, have made parts of the previous strategies obsolete.

- As a result of the tight budget situation, the scientific community is under increasing pressure from the funding agencies and from Congress to set priorities for future research. An observation made by Rep. George $E$. Brown, Jr., former chairman of the House Committee on Science, Space, and Technology, can serve to emphasize this last point: 
As the space program has matured and we have come to understand the full potential for the space sciences, the necessity to set priorities has become essential. In a constrained budget enviromment priority-setting is crucial. . . If scientists fail o [set priorities], science funding may be increasingly decided by "political pork" awarded to localities based on political rather than scientific goals.

For these many reasons, the SSB asked the CSSP-CSTR to undertake the development of a new science strategy to assist science planning efforts for space physics. This report is the CSSP-CSTR response to that request.

The report covers the entire spectrum of subdisciplines that make up the discipline of space physics. It encompasses both big science and little science endeavors and the full spectrum of research methods, including observation (both from space and from the ground), theory, and numerical simulation. Although the emphasis is on the U.S. national program, the science objectives transcend national boundaries, and the study takes into account relevant foreign efforts. While reflecting full cognizance of current and approved programs, in addressing areas of future scientific emphasis the report does not assess any agency's specific implementation plans for future missions or programs. It also does not consider institutional issues (e.g.. universities vis à vis NASA or NSF centers) or other issues associated with the science infrastructure.

The subject matter of the present report overlaps and complements that found in several recent National Research Council science strategy reports. First, the CSSP and CSTR acknowledge and have taken into consideration the work of the Solar Astronomy Panel of the NRC Astronomy and Astrophysics Survey Committee ${ }^{10.11}$ and of the recently completed study of the impact of solar processes on the terrestrial environment. ${ }^{12}$ The committees" consideration of cosmic-ray physics is more focused on the plasma physical aspects of that field than is the concurrent study by a panel of the Board on Physics and Astronomy that emphasizes the astrophysical context. ${ }^{1.3}$ Perhaps the greatest overlap with the present study is evident in one recently completed by the Committee on Lunar and Planetary Exploration (COMPLEX). ${ }^{1+}$ which reviewed the status of magnetospheric and atmospheric research for each of the planets as well as the small bodies of the solar system. Although the CSSP-CSTR study is more terrestrially oriented, the committees recognize that comparison of the similarities and differences between the phenomena observed in different settings can yield greater additional insight into the fundamental atmospheric and plasma processes and can serve as a cross-check on the interpretation of the observations of any single body. The CSSP and CSTR concur with and support the relevant findings and recommendations of those other studies and have considered their conclusions in the process of determining the strategy for space physics.

Finally, the committees note that although the report often speaks in terms of research to be carried out during the coming decade, it is not possible to provide a realistic, detailed schedule, especially in view of the present volatility of science budgets in all agencies. The CSSP and CSTR can only urge that the program outlined here be implemented as rapidly and as vigorously as possible.

\section{NOTES}

1. Board on Global Change. National Research Council, Solar Influences on Global Changer. National Academy Press. Washington. D.C., 1994.

2. Barnes. P.R.. and J.W. Van Dyke. "Potential Economic Costs from Geomagnetic Storms." IEEE Spectrum. March. 1990.

3. The CSTR-CSSP is currently developing a research briefing report on the lopic of these "space weather" causes and elfects

4. Space Science Board. National Research Council, Solar-System Space Physiow in the 1980's: A Resecarch Strategy. National Academy Press. Washington. D.C.. 1980

5. Space Science Board. National Rescarch Council. An Implementation Plan for Priorities in Solar-System Space Physics. National Academy Press. Washington. D.C.. 1985

6. Geophysies Research Board. National Research Council, Solar-Terestral Research for the 1980 's (Herbert Friedman and Devrie Intriligator, co-chairs), National Academy Press. Washington, D.C.. 1981.

7. Board on Atmospheric Sciences and Climate. National Research Council. Numimal Solar-Terrestrial Researith Program (Devrie Intriligator. chair). National Academy Press. Washington. D.C.. 1984.

8. Space Studies Board. National Research Council. Assessment of Programs in Seldor and Spate Phesies-1991. National Academy Press. Washington. D.C.. 1991 .

9. Board on Atmospherie Sciences and Climate and the Space Studies Board. National Research council, A Space Phwicx Parcedes: Why

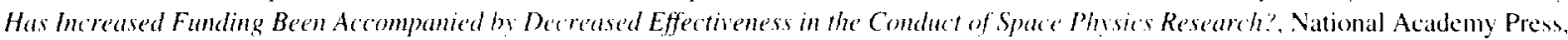
Washington. D.C.. 1994.

10. Buard on Physics and Astronomy. National Research Council, The Dectate of Disconery in Astronom and Astrophwsics. National Acaldemy Press, Washington, D.C., 1991 
11. Board on Physics and Astronomy. National Research Council. Working Papers: Astronomy and Astrophesies Panel Reponts. National Aciademy Press. Washingten. D.C.. 1991.

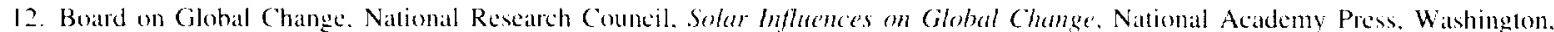
D.C.. 1994 .

13. Board on Physics and Astronomy. National Research Council. Opportumities in Cosmie-Ras Phsies and Astropheses. National Academy Press. Washington. D.C.. 1995

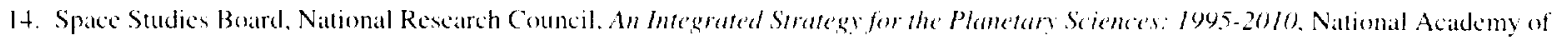
Sciences. Washinglon, D.C., 1994 



\section{Part II}

\section{The Key Topics in Space Physics Research}

Part II of this report discusses five key topics that the committees believe must be addressed by the space physics community in the coming decade. No attempt has been made to prioritize the topics themselves; they are all important. The CSSP and CSTR do, however, prioritize the approaches to trying to answer each topic's major questions. Although the report covers what the committees consider should be the major emphases in the field, there must also be a broad spectrum of other types of research, most of which could be classified as "small science," that may in turn lead to the major thrusts for the following decade.

Several aspects of each of the key topics are considered, including:

- The importance of the topic;

- The current state of knowledge;

- The principal outstanding questions or unproved theories;

- The extent to which the currently approved programs are expected to address the outstanding questions. This assessment is based on the optimistic assumption that the entire currently approved program will be successfully completed - that those NASA space missions that have been approved for a new start will be launched and will operate successfully and that current NSF initiatives will be funded to successful completion; and

- The efforts required beyond the currently approved program, with focus on the next decade. Although the current program can be expected to acquire the new data or insights it was designed to obtain, it will also lead to a new set of questions that had not been foreseen. Those new questions are necessarily beyond the scope of the present study. It is, however, possible to recognize the known limitations of, or gaps in, the currently approved program and to sketch out what needs to be done next. It is obvious that any science strategy must be frequently reviewed and updated to take account of recent events.

In describing the recommended future directions, the CSSP and CSTR have considered what needs to be measured, to what accuracy, and, in some cases, with what new or emerging technology. The committees do not, however, address implementation issues such as whether something is best done with a small Explorer or a large mission, nor the temporal sequence of new space missions. Such plans are the responsibility of the implementing agencies and are more subject to the political process than is the establishment of scientific objectives, which are the focus of this report.

Before each of the key topics is discussed, an overarching statement is required about the role of theory and numerical simulation. The importance of theoretical research in space physics has been emphasized repeatedly in NRC studies.' This type of research must not be separated from the observational programs. It must continue to be a central element associated with every single one of the five key topics, in both the immediate and the more distant future. Theory is what connects the measurements to scientific understanding. The committees encourage 
and expect to see an evolution in the level of sophistication of theory and numerical simulations. The growing availability of supercomputers and massively parallel computers will allow the development of increasingly realistic magnetohydrodynamic models extended to three dimensions. For many problems, it is necessary to go beyond quasilinear theory and come to grips with strongly nonlinear effects; new methods and even new languages are being developed to treat those nonlinearities.

The presentation of the key topics arbitrarily starts at the center of the Sun. progresses through the solar atmosphere and solar wind to the Earth's magnetosphere and atmosphere, and then concludes with the cosmic rays entering the solar system from the galaxy and the universe.

\section{NOTE}

1. See. for example. Board on Atmospheric Sciences and Climate and the Space Studies Board, National Research Council, A Space Physics Paradox: Why Has Increased Funding Be'n Accompanied by Decreased Effectiveness in the Condact of Space Physics Re'search". National Academy Press, Washington, D.C., 1904: and Space Studies Board, National Research Council. Assessment of Programs in Solar and Space Physics-1991, National Academy Press, Washington, D.C., 1991. 


\section{Mechanisms of Solar Variability}

\section{SCIENTIFIC BACKGROUND}

The Sun is observed to be a variable star on nearly every time scale from milliseconds to centurics and, according to theory, over much longer times as well. The quest to understand and forecast solar variability is both basic science and strategic science. It is basic science because the Sun is the touchstone for theories of stellar structure and evolution, magnetic activity, and astrophysical dynamos. It is strategic science because solar variability demonstrably affects our everyday lives. As the nearest star, the Sun provides a wide range of opportunities to develop and test theories in such diverse areas as neutrino physics and particle acceleration.

During the declining phase of the last solar activity cycle (1980 to 1986), the ACRIM experiment on the Solar Maximum Mission (SMM) indicated that the Sun's total radiative output diminished by $0.1 \%$, while the ERB/ Nimbus-7 data indicated that the output diminished by nearly $0.2 \%$. The percentage reduction at ultraviolet wavelengths was 10 to 1000 times larger. The NRC study Solar Influences on Global Change (the Lean report) concluded that such changes in the Sun's radiative output directly modify the global surface temperature, the ozone layer, and the structure of the middle atmosphere. Those solar-induced effects must be included when researchers weigh observational evidence of anthropogenic influences such as greenhouse gases.

Researchers have not directly observed solar irradiance variations on longer time scales. However, space physicists do know that the Sun has shown greater swings of activity in the past than it has recently. There were extraordinarily few sunspots during the period from 1645 to 1715 (the so-called Maunder Minimum). If the Sun`s radiative output followed sunspot number then as it does now, the radiative output was about $0.25 \%$ lower during that period, which coincided with the Little Ice Age in Europe and North America. Indirect indicators of solar activity such as ${ }^{14} \mathrm{C}$ in tree rings and ${ }^{10} \mathrm{Be}$ in ice cores strongly suggest that periods similar to the Maunder Minimum have occurred repeatedly over the last few millennia. Solar activity has also undergone extended periods of enhancement that are associated with climatic warming. During the so-called Grand Maximum of 1000 to $1200 \mathrm{AD}$, agricultural conditions in Northern Europe were good but the American Southwest suffered extended droughts. How typical is this behavior? Other stars of mass, age, and spectral type similar to those of the Sun have shown $0.5 \%$ brightness changes in as little as 5 years.

The theory of stellar structure and evolution as applied to the Sun and other stars stands as one of the major achievements of 20 th-century astrophysics. Yet, until recently, the connection of this theory with observation has been made only through gross properties such as stellar mass, radius, and luminosity, with no direct information from the interior. Over the last two decades, two new types of solar observations-neutrino-flux measurements and helioseismology - have changed this picture profoundly and challenged "standard" models of the Sun"s interior and its magnetic dynamo. These challenges must be taken into account when space physicists study solar variability hecause all forms of solar activity are thought to derive ultimately from the interaction of magnetic 
fields with differential rotation and turbulent convection in the solar interior and atmosphere. When refined and extended, the methods of helioseismology and neutrino-flux measurement will be powerful tools for solving the problems they have uncovered.

The generation of energy in the core of the Sun produces neutrinos by several different nuclear reactions. For nearly two decades, high-energy neutrinos from the formation and decay of ${ }^{x} \mathrm{~B}$ have been observed through their capture by the ${ }^{37} \mathrm{Cl}$ isotope in the Homestake gold mine in South Dakota. The observed capture rate in that experiment is roughly three times smaller than predicted. Neutrinos from the more fundamental proton-proton reaction that begins the fusion process have lower energy and are more difficult to detect. The newer GALLEX experiment in Italy and the SAGE experiment in Russia both use the ${ }^{71} \mathrm{Ga}$ nucleus to detect low-energy neutrinos, including those from the proton-proton reaction. ${ }^{2}$ The combined results from all experiments cannot be reconciled with standard solar models and strongly indicate that the neutrinos are altered in their travel from the solar core to Earth. The reconciliation may require fundamental changes in our ideas of solar structure or neutrino physics.

Helioseismic measurements have constrained solar models by precisely locating the boundary between the Sun's convection zone and its radiative interior. Figure 3 shows a schematic representation of the structure of the Sun together with a recent example of helioseismic measurements that quantitatively probe its interior. For 30 years, phenomenological and kinematic models of the solar dynamo have assumed a pattern of differential rotation in the convection zone that is now ruled out by helioseismology. As a result. dynamo theory is in ferment; current theoretical activity centers on the possibility that the seat of the dynamo is just below the base of the convection zone. Longer-term and more accurate seismic measurements will allow researchers to investigate many more aspects of solar structure and variability. For example, as the precision of frequency measurements improves and if the low-frequency gravity modes are detected, it will become possible to deduce accurately the near-center temperatures and densities from helioseismology and to compute neutrino fluxes without relying on the theory of stellar evolution.

Researchers have made substantial progress in understanding small-scale convection (granulation) in the outer solar envelope. Benchmark observations were obtained by the SOUP experiment on Spacelab-2, now augmented by observations from ground-based observatories. Many of the observed characteristics of the granulation and the flows associated with convection are impressively reproduced by recent three-dimensional magnetohydrodynamic simulations that include radiative transfer. By contrast, the large-scale aspects of convection and flows in the outer solar envelope are poorly understood. Many numerical models of convection exhibit patterns that resemble giant banana-shaped cells; observations of the surface velocity have thus far shown no trace of such structure but instead show flows referred to as torsional oscillations: latitude-dependent variations in the rotation rate that track the zones of activity. The present observations suffer from a lack of temporal continuity that limits our ability to detect low-amplitude, large-scale structures. The observations necessary to study solar velocity oscillations will also provide an ideal data set for the study of large-scale convection. As was the case for helioseismology, these studies will benefit from extended-duration data sequences that match the Sun's 11-year activity cycle.

Researchers do not know how strong the magnetic fields are in the solar interior, nor to what extent they are filamentary, as they are at the surface. Helioseismology should eventually place useful limits on the strength and uniformity of the internal field, but the present upper limits are too high to discriminate among dynamo models. If strong fields near the base of the Sun's convection zone are the source of surface phenomena such as sunspots and plage regions, then understanding the mechanisms that connect the deep and surface fields is necessary. Helioseismology measurements carried out through a solar cycle would permit the study of subsurface magnetic fields through their effect on the propagation velocities of different oscillation modes.

Local magnetic fields on the solar surface are associated with changes in the solar oscillation spectrum that may be caused by subsurface fields. Strong magnetic fields like those in sunspots absorb more acoustic energy than they emit. Some of the effects are apparent even during the days before a new spot group appears and may be general predictors of activity over time scales of a week to a month.

Although sunspots are the most dramatic manifestation of magnetic fields on the solar surface (Figure 4), even regions where the average field is weak are inhomogeneously dotted with tiny magnetic flux tubes in which the intrinsic field is almost as strong as in sunspots. These flux tubes interact with plasma motions and with each other to constitute the fundamental building blocks of solar activity. They have a characteristic diameter of only about $100 \mathrm{~km}$ (subtending barely 0.1 arc sec at the Earth) and have never been directly observed-although, very recently, the upper end of their size spectrum $(\sim 200 \mathrm{~km})$ may have been glimpsed through the technique of speckle 

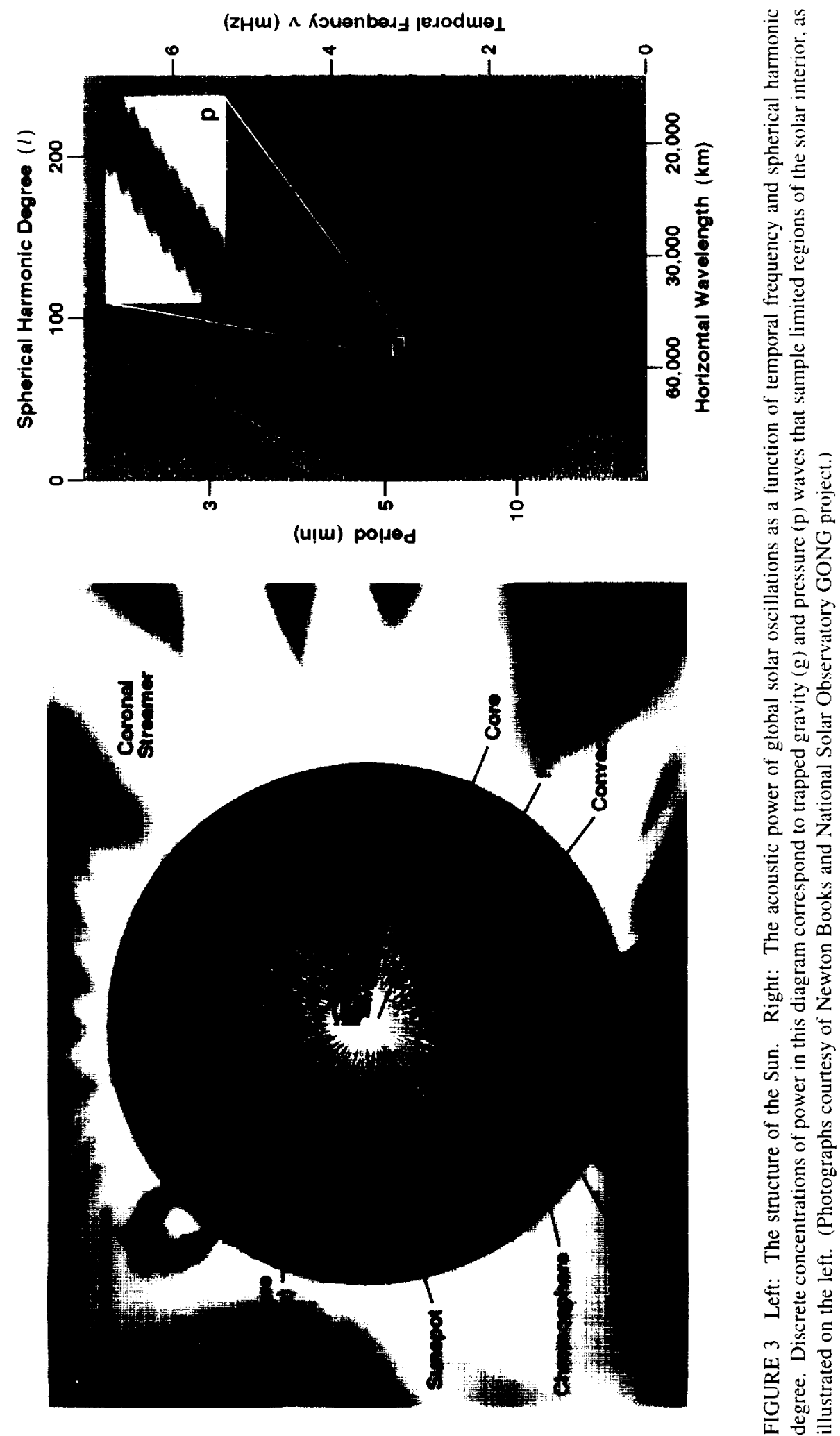


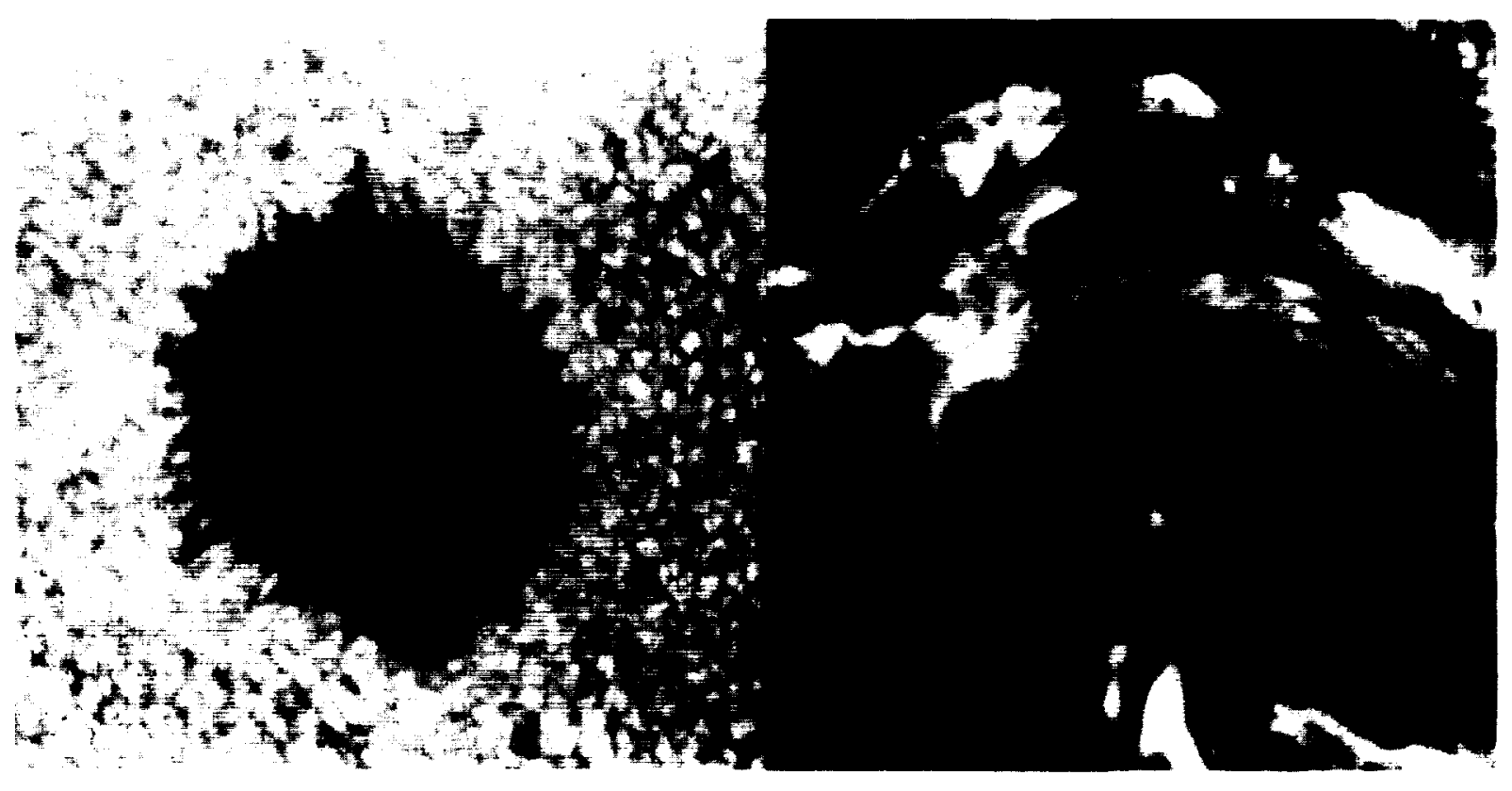

FIGURE 4 Left: The intricate structure of a sunspot in the photosphere. An ever-changing pattern of convection surrounds the spot. Right: A solar active region seen higher in the atmosphere (the chromospheric Ho). Structure of comparable complexity persists even into the solar corona (see Figure 6). (Courtesy of National Solar Observatory and Big Bear Solar Observatory.)

polarimetry. In order to validate and extend our understanding of solar magnetism, it is essential to resolve and study magnetic flux tubes.

Study of the largest individual flux tubes requires observations that achieve a consistent resolution of $150 \mathrm{~km}$ for hours at a time, over angular fields many are seconds on a side-conditions unachievable from the ground, but possible with future space missions. However, both indirect observations and magnetohydrodynamic models show that the physical properties of flux tubes (including their contribution to the solar irradiance) change significantly with size; it is not enough to study only the largest structures. Therefore, the long-term goal remains the one stated by the Solar Astronomy Panel of the NRC's Astronomy and Astrophysics Survey Committee and by the NASA Mechanisms of Solar Variability program: ${ }^{4}$ to achieve $75-$ to $100-\mathrm{km}(\sim 0.1$-arc-sec $)$ resolution both from the ground and space.

The impulsive brightening in visible light that originally defined a solar flare is now recognized as only one component of a complex magnetic reorganization that liberates energy in the form of electromagnetic radiation (from radio waves to gamma rays), energetic particles, and bulk motions. Among the most important types of events in terms of their effect on the magnetosphere are the large-scale coronal mass ejections (CMEs) that have little or no visible-light signature. The shocks preceding the fastest CMEs are capable of producing large fluxes of energetic particles. Solar flares are often associated with the CMEs and are capable of generating impulsive bursts of energetic particles as well as hard $\mathrm{x}$-rays and gamma rays. Neither the trigger mechanisms for CMEs and solar flares nor the particle acceleration mechanisms are known beyond a rudimentary level.

The range in time scale for flares and CMEs (from days during the buildup to milliseconds at the height of outbursts), as well as the range in spatial scale (from $100,000 \mathrm{~km}$ for the size of an active region to $100 \mathrm{~km}$ for the size of the flux tubes), places competing requirements on observation and analysis systems. In order to achieve a coherent understanding of flares and CMEs, space physicists must study both their buildup, by continuously measuring vector magnetic fields in active regions and near large-scale magnetic boundaries, and their release, by measuring their energetic emissions with high angular, temporal, and spectral resolution.

In the last 15 years, several venerable but hitherto unproved notions about flares and mass ejections have been placed on a firm footing by space missions such as P78-1, SMM, and Yohkoh, and by closely coupled groundhased observations (often coordinated through campaigns such as Max '9l and CoMStOC). The importance of 
twisted and sheared magnetic fields (with associated electric current systems) has been confirmed. although much remains to be learned about their evolution and dynamics. The ablation of chromospheric material in response to downward-directed particle beams has been detected and modeled. The topological reorganization of magnetic fields after a flare-compelling evidence of reconnection-has been clearly observed and studied. Even apart from flares, large regions of the corona are seen to restructure on time scales as short as an hour.

In summary, the goals of the study of solitr structure and variability are to understand the basic processes governing the nuclear and rotational evolution of the Sun and, by inference, other main-sequence stars. to understand the physics of neutrino production and propagation, to understand the solar cycle and concomitant variations of the solar radiative output. and to understand solar activity well enough to produce useful forecasts of solar flares, coronal mass ejections, solar energetic particle events, and other forms of impulsive activity. Specific research questions that should be addressed to achieve those goals are as follows:

- What are the mechanisms responsible for variations in the spectral and total irradiance of the Sun and solartype stars?

- What are the large-scale velocity fields inside the Sun as functions of depth. latitude, and phase of the solar cycle?

- What is the structure of the Sun's internal magnetic field?

- How are the internal velocity and magnetic fields related to the surface fields?

- How does convection operate? What is the role of magnetic buoyancy in the convection zone? What is the structure of the transition between the radiative core and the convection zone?

- How is magnetic energy stored and released in the solar atmosphere?

- How are active regions born? How do they evolve and die?

- What is the spectral energy distribution of neutrinos from the solar interior and how can it be explained through solar models?

- What magnetic configurations and evolutionary paths lead to flares and coronal mass ejections?

- What is the physics of solar transients that cause impulsive emissions of radiation, plasma, and high-energy particles?

\section{CURRENT PROGRAM}

The current program seeks to improve our knowledge of the solar interior and solar variability principally through monitoring of the solar irradiance and measurements of solar oscillations. Solar variability is monitored through several experiments on the Upper Atmosphere Research Satellite (UARS), and major experiments are being developed with substantial international collaboration to study solar oscillations. Additionally, the ultimate source of solar energy, the core nuclear reactions, are being studied by international collaborations through the measurement of the neutrino fluxes.

The UARS carries a total solar irradiance monitor (ACRIM) and two ultraviolet spectral irradiance monitors. The Solar and Heliospheric Observatory (SOHO) will measure variations in the total solar irradiance as well as manifestations of solar activity. As the Lean report ${ }^{5}$ emphasized, however, there is a real danger that the crucial cross-calibration of the irradiance measurements will be lost if components of the Earth Observing System do not overlap UARS or SOHO (Figure 5). A key element of the strategy to understand and forecast-rather than just monitor-irradiance variations is to determine precisely, throughout a solar cycle, to what degree the variations are caused by changes in localized surface and subsurface properties and, therefore, how much must be attributed to global changes in the Sun's output. The ground-based RISE program is designed to fill this need, but it is just getting under way and does not have the assurance of long-term funding.

One of the reasons for the continued operation of the Homestake neutrino experiment is the detection of a potentially significant correlation of neutrino flux with solar activity. The newer experiments that detect lowerenergy neutrinos from the proton-proton nuclear reaction allow several possible theoretical interpretations within the present statistical uncertainty. Consequently, continuation of all the experiments will produce an important narrowing of the experimental uncertainty and permit fewer interpretations of the combined results.

The current program in helioseismology comprises al variety of small-scale experiments with different ranges of spatial resolution, sensitivity, temporal continuity, and duration of operation. There are also two large programs 


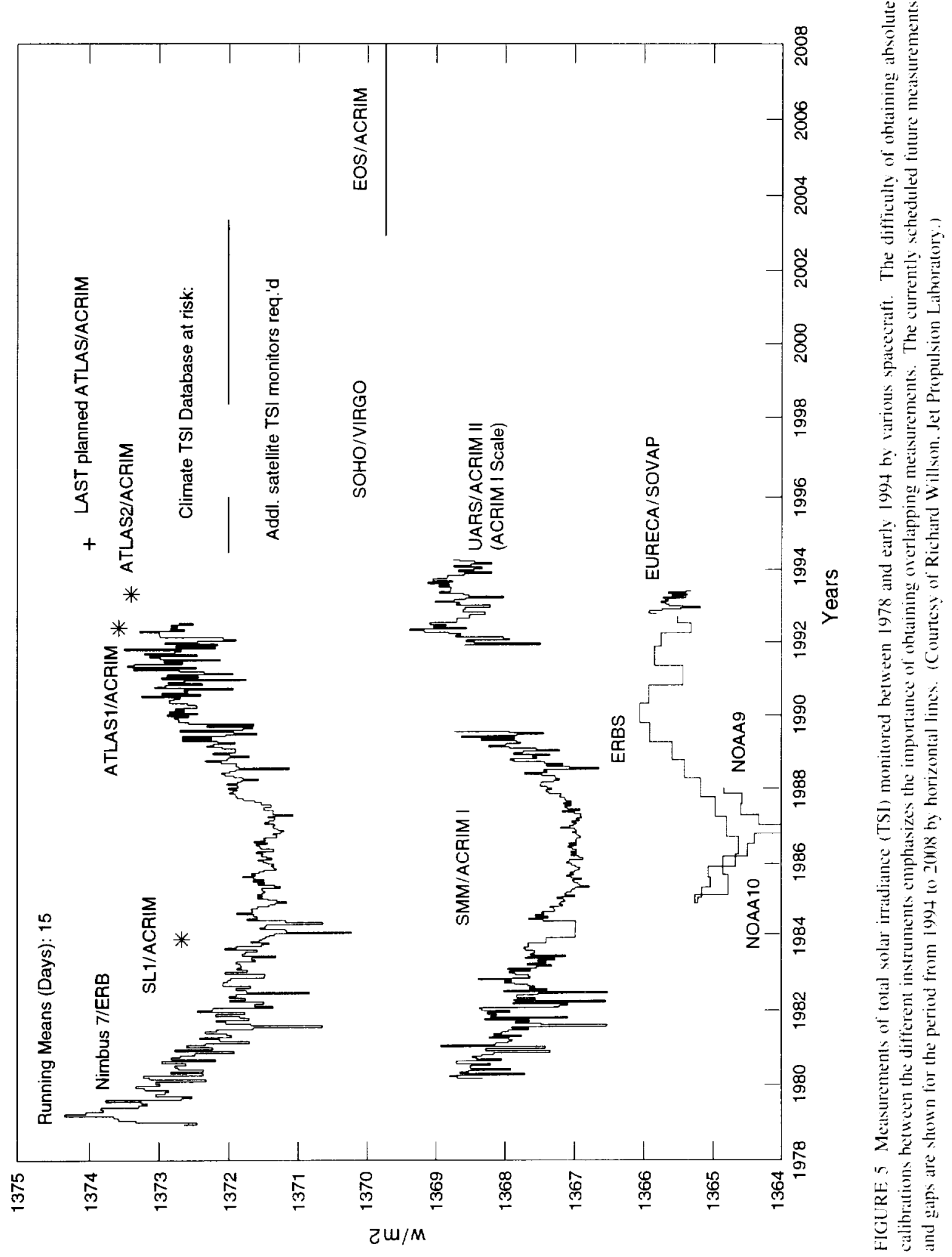


that will reach fruition in 1995: the Global Oscillation Network Group (GONG) project and a suite of three instruments on SOHO.

GONG consists of six observatories regularly spaced around the globe. Because the Sun never sets on it, the network will be able to distinguish oscillations that differ in frequency by only 1 part in 10 billion. GONG will measure nonradial oscillation modes that are characterized by spherical harmonics of moderate degree and will provide the best available temporal continuity for those modes. The ground-based nature of the GONG network permits servicing and upgrading of system components but also introduces some uncertainties due to diurnal trends and the variability of the terrestrial atmosphere at each site. The SOHO instruments will provide high spatial resolution and the most complete determination to date of solar interior structure, especially of convection in the solar envelope, together with stable and sensitive measurements of solar velocities. SOHO also offers the best chance of detecting the elusive low-frequency gravity modes. The improved precision and temporal continuity of velocity measurements to be achieved by GONG and SOHO may permit the detection of large-scale convective flows. This is a secondary objective for both projects, however, and there is a risk that those studies may be omitted for lack of adequate support.

Temporal changes seen in the Sun's acoustic spectrum indicate the desirability of extending observations to include a major fraction of a solar cycle. These changes have been detected in limited ranges of global structure but have not been measured systematically. Both GONG and SOHO are planned for 2 to 3 years of operation. Depending on the efficiency of launch and transfer orbit insertion, SOHO can be extended for an additional 1 to 4 years before exhausting spacecraft consumables, but GONG could operate for a full solar cycle if funding were available.

Although researchers anticipate considerable advancement from the generation of helioseismological experiments soon coming on line, this is a new field of investigation and the experiments have known limitations. None of the experiments uses multiple spectral lines that could permit the study of the vertical structure of the velocities. High-frequency oscillations may play a role in energy transport, but no current experiment has adequate temporal and spatial resolution to study such oscillations. Atomic reference systems have been eliminated from the imaging experiments on both SOHO and GONG, and so their velocity measurements rely on the mechanical stability of their optical systems to provide the velocity zero points. Because only one hemisphere of the solar surface can be measured at a time, the resolution in wave number is fundamentally limited.

Studies of small-scale convection and surface flows require subare-second imaging and extended lime series (an hour or more). Ideally, such observations are carried out above the Earth's atmosphere. However, apart lrom a prospective contribution by the Flare Genesis balloon experiment, ${ }^{6}$ the current program relies on ground-based observatories. which strive for the necessary resolution through a combination of intrinsic image quality (a good site), frame selection, digital postprocessing, and sophisticated techniques, such as speckle imaging and adaptive optics. that show great potential but have not been supported at a level that enables them to make rapid progress.

U.S. participation in the Japanese Yohkoh mission and the European SOHO mission represents two major components of the current program for the study of solar variability and activity. Yohkoh carries a cluster of telescopes that measure $x$-ray emission from flares and the corona. Soft $x$-ray images are often made simultaneously with ground-based maps of the magnetic fields and visible-light structures on the Sun. Particularly rapid progress is being made in this way in mapping the overall structure of flares and the effects of tlares on the corona. The soft $x$-ray spectrometer reveals the temperature, density, and flow velocity of hot, dense plasmas. Yohkoh can also probe particle acceleration sites with a hard $x$-ray telescope (7-arc-sec resolution) and a wide-band $x$-ray/ gamma-ray spectrometer (full Sun). SOHO will also measure atmospheric structure and activity with a diverse complement of instruments.

Two rocket programs have successfully demonstrated the use of normal-incidence optics to obtain narrowhand soft $x$-ray images with high angular resolution. Such instruments will be powerful tools for the study of activity if they can be given a long-duration platform. An imager to be flown on a future NOAA/GOES satellite will provide soft $x$-ray images on a continuous, high-time-resolution. long-term basis, but at much lower angular resolution.

The HIREGS balloon experiment has observed hard x-ray "microflares," nonthermal events up to 100 times weaker in flux than previously detected, but still rising in numbers to the limit of instrumental sensitivity. The likelihood that there is a continuous size spectrum of flares, from the rarest spectacular events to ubiquitous microtlares (and perhaps even smaller "nanoflares"), has given rise to new theoretical ideas about flares and their connection with coronal heating. 
In the gamma-ray regime, the Compton Gamma Ray Observatory (CGRO) provides capability to study events with low spectral resolution and no spatial information. This observatory has detected solar gamma rays with energies up to $1 \mathrm{GeV}$ and has directly measured the energy spectrum of solar neutrons. The CGRO found the highenergy bremsstrahlung emission to be highly collimated along the direction of electron motion. This observation is interpreted to mean that the electrons are trapped in a closed magnetic structure.

Ground-based observations - free of the mass and size constraints of space experiments, accessing a wide spectral range, and able to incorporate the latest technology - remain a cornerstone of solar physics. In addition to special-purpose applications such as magnetographs and helioseismology instruments, general-purpose telescopes (including spectral isolators and detectors) have been the testbeds for new ideas and techniques. For example, frequency-agile radio imaging has provided new information about the magnetic configuration of active regions before and after flares. There has been an explosive growth in infrared observations, enabled by the development of infrared array detectors, with applications ranging from the deepest photosphere to the corona.

Ground-based observations of the solar magnetic field fall into two general categories. Full-disk synoptic measurements emphasize high sensitivity to the line-of-sight field, stable calibration, and continuity over many years. Together with the international sunspot number and the $10.7-\mathrm{cm}$ radio flux, these are among the most widely used data in solar and solar-terrestrial physics. A second category of magnetographs is intended for the detailed study of active regions and magnetic flux tubes. Such instruments emphasize measurements of the full vector field and electric currents, subarc-second angular resolution, good temporal resolution, and precise measurements of the intrinsic field strength using infrared spectral lines. These instruments, most of which are less than 5 years old, represent a major technical and scientific advance over what prevailed a decade ago. However, two major preconditions for understanding surface magnetic fields are still unmet: the ability to study flux concentrations at their intrinsic size scale (corresponding to 0.1 to $0.2 \mathrm{arc} \mathrm{sec}$ ), and continuous measurements at intermediate resolution $(\sim 1$ arc sec) over the full lifetime of an active region.

\section{FUTURE DIRECTIONS}

The natural time scale of the solar cycle dictates that some types of observation must be maintained for decades or longer. This requirement is difficult to meet when budgets and political priorities change on a shorter time scale. Nevertheless, a high scientific priority must be accorded the continuation of modest long-term studies of cycle-dependent phenomena such as magnetic flux, sunspots and plages, coronal brightness, and large-scale velocity patterns. It is also vital to assure the continuity of existing spaceborne irradiance monitoring (total and spectral). The whole-Sun fluxes recorded by the irradiance instruments should be supported by spatially resolved, multisite photometric measurements from the ground or space.

Progress in the analysis of solar interior structure using the methods of helioseismology requires improved precision in the oscillation frequencies and a larger range of detected modes. The details of the most effective strategy to extend helioseismology studies should be determined after GONG and SOHO have been in operation for about a year. However, there is already a compelling case for maintaining GONG through a full solar cycle, with adequate support for the analysis of large-scale flows.

A major advance in the study of energetic events is possible by combining high detector sensitivity with high spatial and spectral resolution. Germanium detectors behind rotating linear masks can provide time resolution as short as 0.01 second with spatial resolution in the arc-second range and enough spectral resolution to reveal dynamics through Doppler distortion of spectral line profiles. This combination yields powerful diagnostics for following the development of the impulsive phase of hard $\mathrm{x}$-ray and gamma-ray flares and for studying energetic particle propagation.

Other frontiers in research on flares and active regions lie in the exploration of poorly known regions of the flare electromagnetic spectrum, such as the infrared and submillimeter domains; in multifrequency radio imaging; in continuous observations of vector magnetic fields and velocity fields in active regions through their entire life cycle; and in acquiring a three-dimensional view of coronal structures and mass ejections.

The complex and small-scale character of the Sun's surface magnetoconvection demands high angular resolution $(0.1$ to 0.2 arc sec), good temporal resolution $(\sim 10 \mathrm{~s})$, accurate polarimetry $(<0.1 \%)$, and the ability to simultaneously measure the magnetic field strength in sunspots and weak-field regions as well as diagnostics of the thermodynamic state of the magnetic and nonmagnetic gas. Current instruments achieve (or, in the case of angular resolution, approach) only subsets of these requirements. Fully capable instruments should be developed 
and fed by telescopes able to supply the requisite photon flux (aperture $>1 \mathrm{~m}$ ). A global network, able to provide 1-arc-sec resolution and 24-hour coverage over a week or more, is needed to characterize the magnetic evolution of active regions over their lifetimes. Adaptive optics and image-reconstruction techniques should be supported to advance the goal of achieving 0.1 -arc-sec angular resolution over a restricted field of view. However, for precision photometry at this resolution over an active-region field of view, balloon or spacecraft systems are required.

Viewing the Sun from more than one solar longitude has several unique and practical advantages. For example, an observing station over the solar east limb would increase the chance of obtaining an extended set of observations beginning with a change in the oscillation spectrum and ending with the appearance of an active region. This is perhaps the best hope for developing the ability to predict the emergence of activity. Other motivations for a global-view array of spacecraft include early detection and warning of solar activity, tomographic reconstructions of the three-dimensional structure of the outer atmosphere, and understanding of irradiance variations. Achieving all of these objectives would require several types of instruments on each spacecraft; simpler missions could address subsets of these goals.

The measurement of neutrino fluxes with different detection systems will provide the most direct observations of the solar core. The gallium experiments, such as SAGE and GALLEX, are especially critical until their statistical uncertainties are reduced to $<10 \%$. Although non-U.S. groups have primary responsibilities for those experiments, the support of U.S. investigators should be given high priority as a good return on investment. The Homestake mine experiment should be continued through the current solar cycle to assess the reality of the reported solar-cycle modulation.

Asteroseismology poses a technical challenge that has great promise for validating and extending our theories of stellar structure and evolution. Current synoptic programs to monitor activity and magnetic fields on late-type stars should be maintained. However, the sample of stars that can be studied with existing facilities is very limited, particularly for the latest types; access to a 4-m-class telescope would expand the sample by an order of magnitude.

The Solar Astronomy Panel of the NRC's Astronomy and Astrophysics Survey Committee ${ }^{7}$ emphasized the need for a major effort to develop observational capabilities in the infrared. For solar physics, infrared wavelengths allow unique diagnostic capabilities for the measurement of magnetic field strength and temperature. The realization of the potential for new discoveries in the infrared will depend on the continued development of infrared technology (such as large-array detectors and tunable narrow-band filters) and, ultimately, a large infrared-capable telescope to provide subarc-second angular resolution in the range from 3 to 10 microns.

It is possible that new insights into two of the more important issues in solar physics - the solar dynamo and magnetic reconnection in solar flares-may emerge from a combination of new laboratory experiments and theoretical research. The potential for the application of laboratory plasma physics to space physics problems has been summarized in reports by the NRC's Plasma Science Committee ${ }^{8}$ and Panel on Opportunities in Plasma Science and Technology. ${ }^{9}$

\section{NOTES}

1. Board on Global Change, National Research Council, Solar Influences on Global Change, National Academy Press, Washington. D.C. 1994.

2. Board on Physics and Astronomy, National Research Council. Neutrino Astrophysics: A Research Briefing, National Academy Press. Washington. D.C. 1995.

3. Board on Physics and Astronomy, National Research Council, Working Papers: Astromomy and Astrophysics Panel Reports, National Academy Press, Washington, D.C.. 1991.

4. The Mechomisms of Solar Variability Program. report of a workshop presented by the Space Physics Subcommittee of NASA's Office of Space Science and Applications, Washington, D.C.. January 7-9. 1992.

5. Board on Global Change. National Research Council, Solar Influences on Global Change. National Academy Press, Washington. D.C. 1994.

6. Rust, D., D. Lohr. G. Murphy, and K. Strohbehn, "The Flare Genesis Experiment: Studying the Sun from the Stratosphere," presented at the AIAA 32nd Aerospace Sciences Meeting and Exhibit, January 10-13, 1994, Reno. Nev.

7. Board on Physics and Astronomy, National Research Council. Working Papers: Astronomy and Astrophysics Panel Repoms. National Academy Press. Washington, D.C., 1991.

8. Board on Physics and Astronomy, National Research Council, Research Briefing on Comtemporary Problems in Plasma Science. National Academy Press, Washington. D.C.. 1991

9. Board on Physics and Astronomy, National Research Council, Plasma Science: From Fundamental Research to Techology Applications, National Academy Press, Washington, D.C., 1995 


\section{The Physics of the Solar Wind and the Heliosphere}

\section{SCIENTIFIC BACKGROUND}

The Sun's outer atmosphere. or corona, is extraordinarily hot (over a million degrees) compared to the visible outer surface. The solar wind and the heliosphere exist because the pressure of the hot corona is too great to be contained by the combination of solar gravity, magnetic forces, and the pressure of the interstellar medium. Observations of stellar $x$-rays show that coronas and winds are pervasive features of late-type stars. As the only stellar-scale plasma system that permits a close-up view and in situ measurements, the Sun and heliosphere must be the guideposts for understanding those general astrophysical phenomena.

Researchers do not yet understand the physical mechanisms that heat the solar corona, although it is generally accepted that magnetic fields must play a central role. X-ray images acquired from space show that much of the corona is dominated by arch-like structures of plasmas confined by the solar magnetic field. The variety and superposition of magnetic structures visible at any time compound the difficulty of resolving issues associated with coronal heating mechanisms. The $\mathrm{x}$-ray observations also show a strikingly filamentary and restless corona (Figure 6); flares and mass ejections (see Chapter I, "Mechanisms of Solar Variability") are but one component of the pervasive variability. In addition. there are regions, known as coronal holes, that appear as dark areas in $x-$ ray images of the Sun. The magnetic fields from coronal holes are carried into interplanetary space by the solar wind. X-ray observations also reveal widespread tiny active regions. called coronal bright points, that may play a role in heating the corona and accelerating the solar wind. On the other hand, observations at 1 AU show the preponderance of magnetohydrodynamic waves, in particular, Alfvén waves, of solar origin. Such waves may play a very important role in the acceleration of the solar wind.

In the early 1970s coronal holes were identified as the source of the high-speed, quasi-stationary wind. but the solar source of the low-speed, quasi-stationary wind is still being debated. The speed and the fluxes of mass and energy of the low-speed wind are consistent with acceleration by thermal pressure gradients in the corona, but some additional push is required to generate the high-speed wind from coronal holes. Theories abound, but the relative importance of waves and of jets of particles, perhaps generated in bright points or in myriad tiny flare-like events. is not yet known. It is considered likely by some theorists that the unknown mechanisms that heat the corona and that accelerate the wind in coronal holes are very closely linked.

This quasi-stationary solar wind is intermittently interrupted by the passage of coronal mass ejections (CMEs). CMEs are distinet plasma structures in interplanetary space that open and carry out magnetic fields from previously closed regions of the corona. Since the magnetic field is not observed to build up in interplanetary space, there must be some process closing off open flux; this opening and closing is an active area of research. About one-third of all CMEs are sufficiently fast to drive transient interplanetary shocks; it is these fast CMEs and the draped and compressed magnetic fields ahead of them that cause nearly all of the largest geomagnetic storms at 


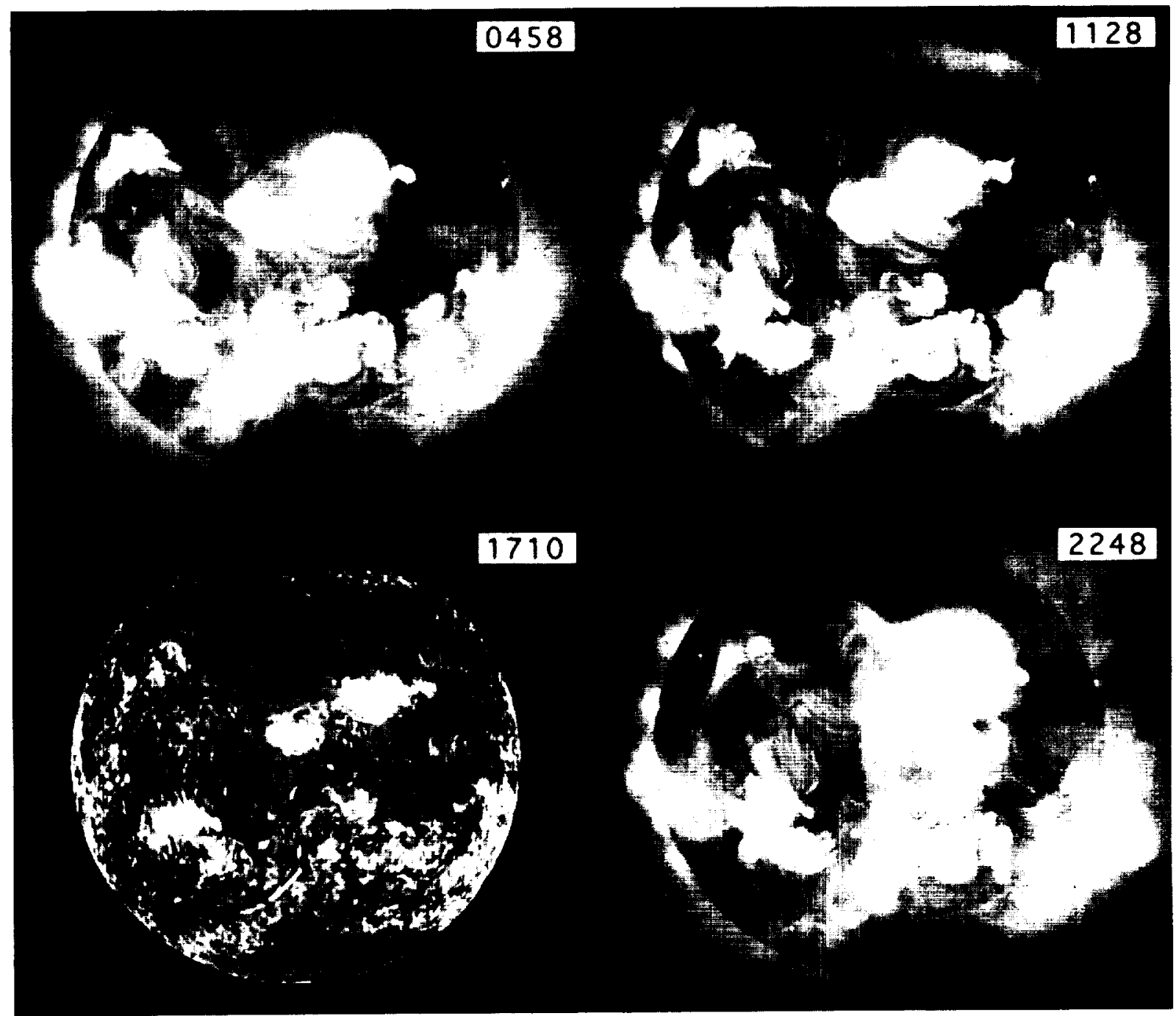

FGURE, 6 Clockwise from upper left, the development of coronal structure observed by Yohkoh's soft x-ray telescope on November 12,1991. A bright magnetic arcade forms at location $A_{1}$ over a period of a few hours: a transient jet-like feature appears at location J. The kwer left panel is a ground-batsed image (negative print) of neutral helium absorption that reveals structural connections between the chromosphere and the corona-for example, the "footpoints" of the arcade extending to the right of $\mathrm{A}_{2}$ as seen in $\mathrm{x}$-rays (upper right panel) form a bright band in the helium image. (Courtesy Yohkoh team and K.L. Harsey, National Solar Observatory.)

Earth. Unfortunately. it is not yet possible to predict the beginning of CMEs from signatures in the preexisting coronal structure.

The highly variable ion composition of the solar wind is another unresolved puzzle. The relative abundance of helium in the solar wind ranges from essentially zero $10 \sim 40 \%$. In some types of solar wind flows the abundances of the elements depend on their first ionization potentials. whereas that effect is less discernible in the wind from coronal holes. The heavy elements are also observed to flow with speeds exceeding the proton speed. indicating the presence of a poorly understood physical mechanism that preferentially accelerates heavy ions relative to protons. Taken together, the heavy ion abundances, their charge states, and their speeds and temperatures must contain clues. currently undeciphered, to the processes responsible for heating the corona and acceleratting the wind.

As the wind llows out into the heliosphere (ligure 7). the properties such as the densities, velocities, tempera- 


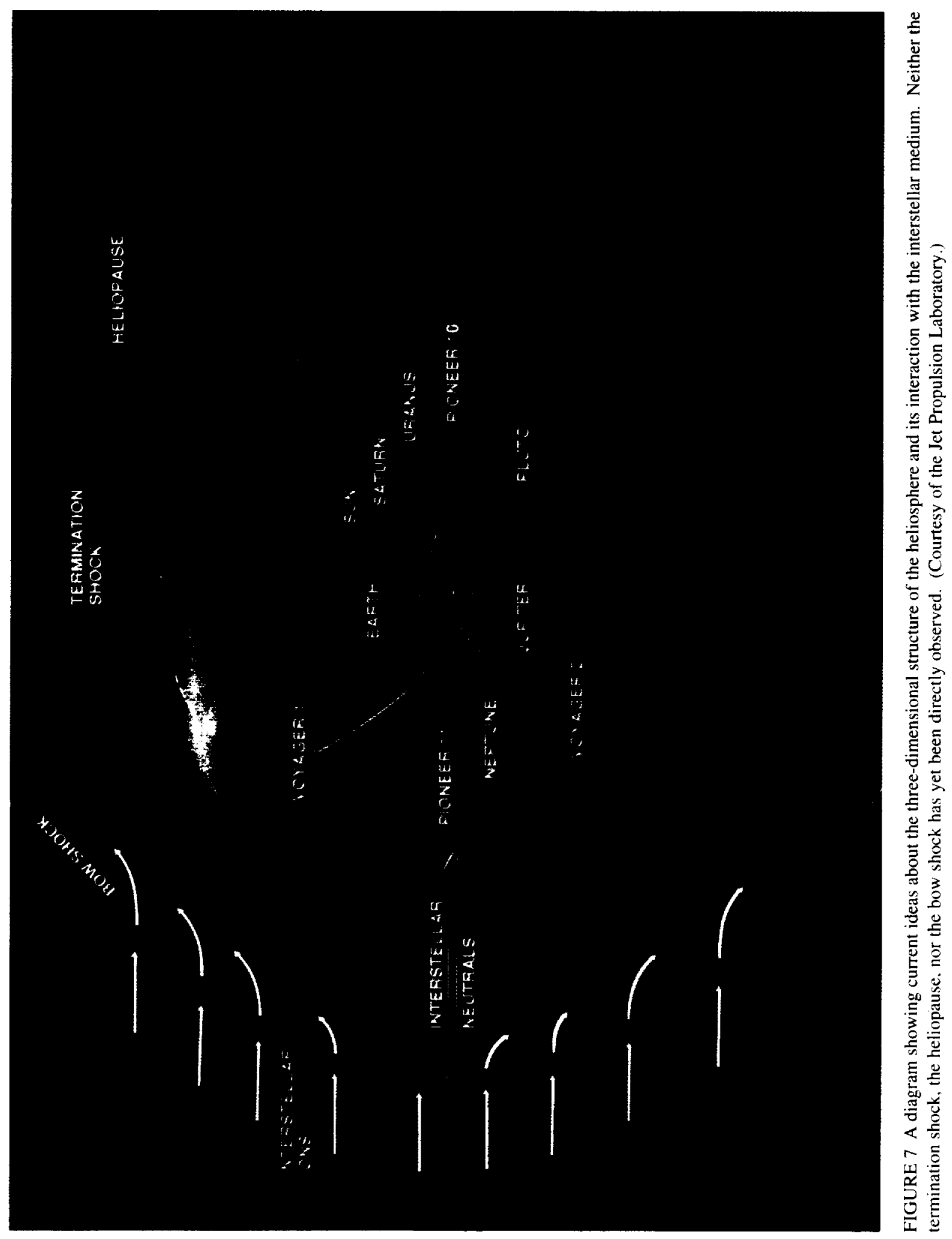


tures, anisotropies, heat fluxes, beams, and other nonthermal characteristics of the ions and electrons evolve. Several factors have been invoked to explain why the temperature of the solar wind cools off with solar distance less slowly than adiabatically. Conservation of the particles' magnetic moments during the expansion, the pickup of newly ionized interstellar gas, and other processes result in unstable configurations that lead to the generation of waves and wave-particle interactions. The cooling rate may be controlled by a turbulent cascade of energy from longer to shorter scales. A related debate concerns the source of interplanetary turbulence: Is it caused principally by velocity shears or by the nonlinear evolution of magnetohydrodynamic waves? The very large scale of the heliosphere provides a unique opportunity to study those specific plasma microprocesses in relative isolation and with minimal confusion by boundary effects.

Although it has been known for 40 years that the flux of galactic cosmic rays in the inner solar system varies roughly out of phase with the solar activity cycle, the dominant cause of the modulation is still being hotly debated. There are sets of observational data that support a diffusion/convection mechanism, and other sets of observational data suggest at other times and other places within the heliosphere the principal mechanism may be particle drifts in the large-scale interplanetary magnetic field.

Finally, heliospheric physicists are poised to enter a new and exciting era of exploration and discoverynamely, direct observation of the interaction of the heliosphere with the local interstellar medium, whose properties are very poorly known. As shown in Figure 7, two or three boundary structures are expected in the region where the solar wind interacts with the interstellar plasma: a heliopause separating the two plasmas, a termination shock where the solar wind is decelerated from a supersonic to subsonic state, and perhaps a bow shock in the interstellar plasma. The Voyager spacecraft have detected low-frequency radio signals not seen in the inner heliosphere. One interpretation is that those waves may be generated by plasma processes at the heliopause. Neutral interstellar atoms are free to stream into the heliosphere where they become ionized and are then carried outward as pickup ions in the solar wind. Acceleration of the pickup ions leads to a population of energetic particles called anomalous cosmic rays, but it remains to be seen how much of the acceleration occurs at the termination shock versus how much occurs upstream of the termination shock, either stochastically or by interplanetary shocks. Determination of the strength of the termination shock and the extent to which it accelerates particles to high energies will also serve as a test of theories that some of the galactic cosmic radiation may be accelerated in the termination shocks of certain types of stars. It is hoped that the Voyager spacecraft will cross the termination shock in the next few years to a decade and will leave the heliosphere sometime during the next few decades.

In summary, the goals of heliospheric physics are to understand why the Sun and many stars have coronas and winds, what physical processes occur in the large regions dominated by the winds, and how the winds interact with the interstellar medium. Specific research questions raised by recent observations of the solar corona and the heliosphere are as follows:

- How does the energy transported from the solar interior heat the corona? How is the magnetic structure of the corona related to its thermal and dynamic evolution? Are coronal bright points an important source of heat? Is coronal heating caused by a theoretically predicted spectrum of microflares and nanoflares that are too small to be seen with current observational capability? Is the absorption of magnetohydrodynamic wave energy important, and if so, how and where are the waves generated?

- What are the three-dimensional topologies of coronal structures? How and where do the magnetic fields in the heliosphere open and close?

- What processes are responsible for the acceleration of the quasi-stationary solar wind? What are the roles of jets, hydromagnetic waves, and bright points? What effect does the magnetic structure have on the acceleration? Where does the slow solar wind originate, and why is it slower than the fast wind from coronal holes?

- What clues about the mechanisms responsible for coronal heating and solar wind acceleration exist in the charge distributions and the elemental composition of solar wind ions?

- How do the properties of the fields and particles in the heliosphere depend on solar distance, latitude, and longitude (with respect to the Sun's motion through the local interstellar medium) and on solar activity? What are the origins, structure, and evolution of plasma waves and turbulence? What is the role of pickup ions for the dynamics and properties of the solar wind? Where and how are the pickup ions accelerated to become the anomalous cosmic rays? How do shocks develop and interact and accelerate particles? How do energetic particles propagate throughout the heliosphere, and what are the relative roles of diffusion/convection and drifts? 
- What are the natures and locations of the heliospheric boundary structures? What are the properties of the local interstellar medium".

\section{CURRENT PROGRAM}

The principal approach to studying coronal heating and solar wind acceleration is to make coordinated multiwavelength observations at different radial distances along the same streamline, starting from the source region at the solar surface and extending into interplanetary space. Such observations are the goal of several current and planned efforts. Yohkoh images the multimillion-degree coronal plasmas at $x$-ray wavelengths. Spartan 201 is a shuttle payload with instruments for remote diagnosis of the properties of the solar wind in the region within 10 solar radii of the solar surface. SOHO will map the solar disk and the corona out to 30 solar radii. The space-based data are supplemented and extended by rocket, balloon. and ground-based observations of the solar surface, the inner white-light corona, solar radio emission, and the scintillation of radio sources (both natural and spacecraft) caused by fluctuations in the corona and the solar wind.

The properties of the solar wind are being and will be measured by near-Earth spacecraft (IMP-8, followed by Wind. and then ACE) and by Ulysses at high solar latitudes. Although ICE (ISEE-3) is available for low-latitude measurements at a longitude far removed from Earth, its operations and data analysis have been terminated. Four current and planned spacecraft (Ulysses, Wind, SOHO, and ACE) carry new-generation instruments capable of determining the mass and charge of solar wind ions. Combining those in situ measurements with remote sensing of conditions at the coronal source of the wind will help address questions about the wind's acceleration and elemental fractionation.

Heliospheric plasma processes and the three-dimensional structure of the heliosphere are addressed by Pioneer 10 (in the ecliptic near $58 \mathrm{AU}$, headed down the heliospheric tail at $2.6 \mathrm{AU} /$ year). Voyagers 1 and $2(\sim 54$ and $40 \mathrm{AU}$, headed into the interstellar wind at midlatitudes with speeds of $>3$ AU/yr). Ulysses (in a high-inclination eccentric orbit reaching out to Jupiter and passing close to the solar poles), and nearr-Earth spacecraft such as IMP8. Wind, and ACE (see Figure 7 and, in Chapter 3. Figure 10). The near-Earth fluxes of energetic particles are also monitored by additional Earth satellites (e.g., SAMPEX, GOES, and DMSP) as well as by ground-based neutron monitors sensitive to galactic cosmic rays and the highest-energy solar particles. The Voyagers are well instrumented for studying the evolution of waves and turbulence, the modulation of galactic cosmic rays, and the acceleration of anomalous cosmic rays and how those processes depend on distance from the Sun and on changes driven by the solar activity cycle. If current estimates of the heliospheric structure are correct, the Voyagers are expected to survive long enough (until 2015) to observe the termination shock and perhaps also the heliopause.

Ulysses adds a third spatial dimension to heliospheric physics. First. the three-dimensional magnetic and plasma structure of the heliosphere will finally be revealed. The minimization of effects due to velocity shears and stream interactions allows the study of processes such as the generation and evolution of turbulence, wave-particle interactions, and energetic-particle transport. Ulysses data will also be used to choose between different theoretical models of the modulation of galactic cosmic rays.

\section{FUTURE DIRECTIONS}

The present configuration of the Voyagers. Pioneer 10. Ulysses. and near-Earth spacecraft is unique, and there are no plans to deploy a second set of such well-distributed heliospheric observatories. Because many of the critical questions listed above concern the large-scale structure of the heliosphere over the full range of solar distances. latitudes, and activity, operating the Voyagers and Pioneer 10 as long as technically feasible and operating Ulysses throughout its second polar passage at the solar maximum are the committees' highest priority for heliospheric research. In addition. Cassini cruise science would allow the first measurements of some solar wind parameters (e.g., pickup ions and electron heat flux) beyond the orbit of Jupiter. Vigorous coordination of the different heliospheric data sets, with each other and with remote sensing of the solar corona by Yohkoh. Spartan 201, SOHO, and ground-based methods, together with the inclusion of guest investigators with different backgrounds and insights. would greatly enhance the scientific yield.

Many of the current and planned studies of the corona not only image its topology. but also provide data from which some properties of the coronal plasma can be inferred, including the lemperatures, densities, and flow speeds of different species, such as electrons, protons, and heavy ions. The calculated parameters, however, are 
subject to large uncertainties because of the highly structured nature of the solar corona and the superposition of plasmas with different temperatures and densities along the line of sight. Only for long-lived coronal features ean observations of the rotating Sun alleviate the problems involved with line-of-sight effects.

One way to reduce the uncertainties and to obtain "ground truth" for the remote sensing methods is to make in situ measurements over the distance range from the $0.3 \mathrm{AU}$ perihelion of the Helios spacecraft down as close as possible to the Sun (within a few solar radii). Making such observations poses formidable technical challenges. If a cost-effective method for exploring this region can be found, it would surely command very high priority. Simultaneous remote sensing of the corona would greatly enhance the value of the in situ measurements.

Another approach to surmounting line-of-sight problems is to obtain tomographic, three-dimensional observations of the Sun by remote sensing from two or more solar longitudes. Observations of the solar corona from a longitude $\sim 90^{\circ}$ from Earth would also allow measurement of the masses and velocities of coronal mass ejections heading Earthward that may cause geomagnetic disturbances a few days later. Other applications of a longitudinally distributed set of spacecraft are discussed in Chapter 1, "Mechanisms of Solar Variability."

Further observations are required to determine which among many hypothesized mechamisms transport energy from the solar surface into the chromosphere and corona. One set of ideas centers on continual magnetic reconnection and fine-scale activity ("nanoflares"). Magnetohydrodynamic waves represent another family of possibilities; theoretical studies suggest particular attention to waves with periods from 1 to $10 \mathrm{~s}$.

The coronal magnetic field is usually estimated by extrapolating the field measured at the solar surface. Direct measurement techniques are not well developed. Estimates derived from radio observations depend on assumptions about the emission mechanism and independent information about plasma density and temperature. Another approach is to infer the field from the linear depolarization (via the Hanle effect) of ultraviolet radiation by neutral hydrogen in the corona, again combined with independent estimates of thermodynamic plasma parameters. Given the importance of the plasma and field parameters in the corona for solar wind models, it is extremely important for future programs to reduce the uncertainties and the model dependence of such measurements.

The instrumentation already launched on Galileo and under development for the Cassini mission is entirely appropriate to obtain data at solar distances intermediate to Earth and the Voyagers during those missions" interplanetary cruise phases; however, there are no current plans to do so. Such cruise data would substantially enhane synoptic coverage of the heliosphere, thus increasing the return on investments already made in those programs. Cruise science should also be included on other future missions that explore regions of the heliosphere remote from the Earth.

Because the possibility of in situ observations of the interstellar medium was not considered in the design of the instruments on the Voyagers, those spacecraft cannot make many of the measurements required to fully characterize the properties of the local interstellar medium and its interaction with the heliosphere. A space mission optimized for the study of the outer heliosphere and the interstellar medium, including the plasmas, cosmic rays, and neutral gas, would greatly enhance our understanding of how the heliosphere interacts with its galactic environs. In addition. none of the interplanetary spacecraft mentioned ahove includes instrumentation for studying phenomena that may depend on the sign of the particle's electric charge. The technology to do so through the study of cosmic-ray electrons and positrons exists but awaits a spaceflight opportunity.

Missions to the innermost heliosphere and the interstellar medium are technically very challenging. The mass that can be delivered for missions of reasonable duration is severely limited by the capability of propulsion systems. Thus, realization of those objectives can be advanced by a program to develop a new generation of very small but still highly capable spacecraft and instruments. 


\section{The Structure and Dynamics of Magnetospheres and Their Coupling to Adjacent Regions}

\section{SCIENTIFIC BACKGROUND}

When the solar wind reaches a magnetized planet such as Earth, this collisionless plasma interacts with the magnetic field, initiating a complex process through which the solar wind can ultimately influence the upper reaches of the atmosphere. The elements of the magnetosphere and its space environment are presented schematically in Figure 8. The streaming solar wind compresses the magnetosphere on the dayside and stretches it out on the nightside in a long anti-sunward tail structure. The interaction between the solar wind, the magnetosphere, and the atmosphere produces a number of plasma and magnetic field structures, including the magnetopause, magnetosheath, boundary layers, cusp region, plasmasphere, ring current, plasma sheet, magnetotail, and magnetotail lobes. Each of these regions maps along magnetic field lines into the atmosphere-ionosphere system and is the origin of energetic inputs that perturb the state of the plasmas and neutral gases. The transport of mass, momentum, and energy into and through magnetospheres and ionospheres is a fundamental yet poorly understood process. The study of the coupling between magnetospheres and ionospheres can be characterized as a synthesis of in situ data collected over a period of years into an intriguing picture of a basic astrophysical process. Yet space-time ambiguities and the problems associated with having only single or limited multipoint observations to study a large and highly dynamic system have resulted in a view of the magnetosphere-ionosphere system that is necessarily schematic. A global perspective requires that the local small-scale plasma processes be placed in the context of the large-scale system, and ideally, provides a connection between small-scale processes occurring simultaneously in widely separated parts of the magnetosphere-ionosphere system.

The magnificent images of vast astrophysical plasma systems, a familiar example being the Crab nebula, clearly laced with magnetic fields, hot gasses, and highly energized charged particles, have captured the interest of both the public and scientists. However, those images and spectral data are the only means of studying the complex processes operating in those objects, because in situ measurements in the nebula are not possible. In contrast, quite the opposite condition holds in the case of planetary magnetosphere-ionosphere systems. Over the past 38 years, many measurements have been made of the constituent gases, fields, and plasmas within the Earth's and other planets' magnetospheres. However, with only a few exceptions. the global structures of those astrophysical plasma systems have remained invisible, and the external influences on and interrelationships between the highly differentiated regions of those systems have been only partially understood.

Computer modeling and simulations are a valuable aid to deducing the global structure of the magnetosphereionosphere system from the point-by-point observational data. Although these models are invaluable for providing a global setting for local satellite measurements and suggesting directions for future studies, there are not yet global observations to assess the accuracy of the data syntheses and the models. The first step in improving this situation has been the use of satellite auroral images (such as those from DMSP. Dynamics Explorer. Viking, and 


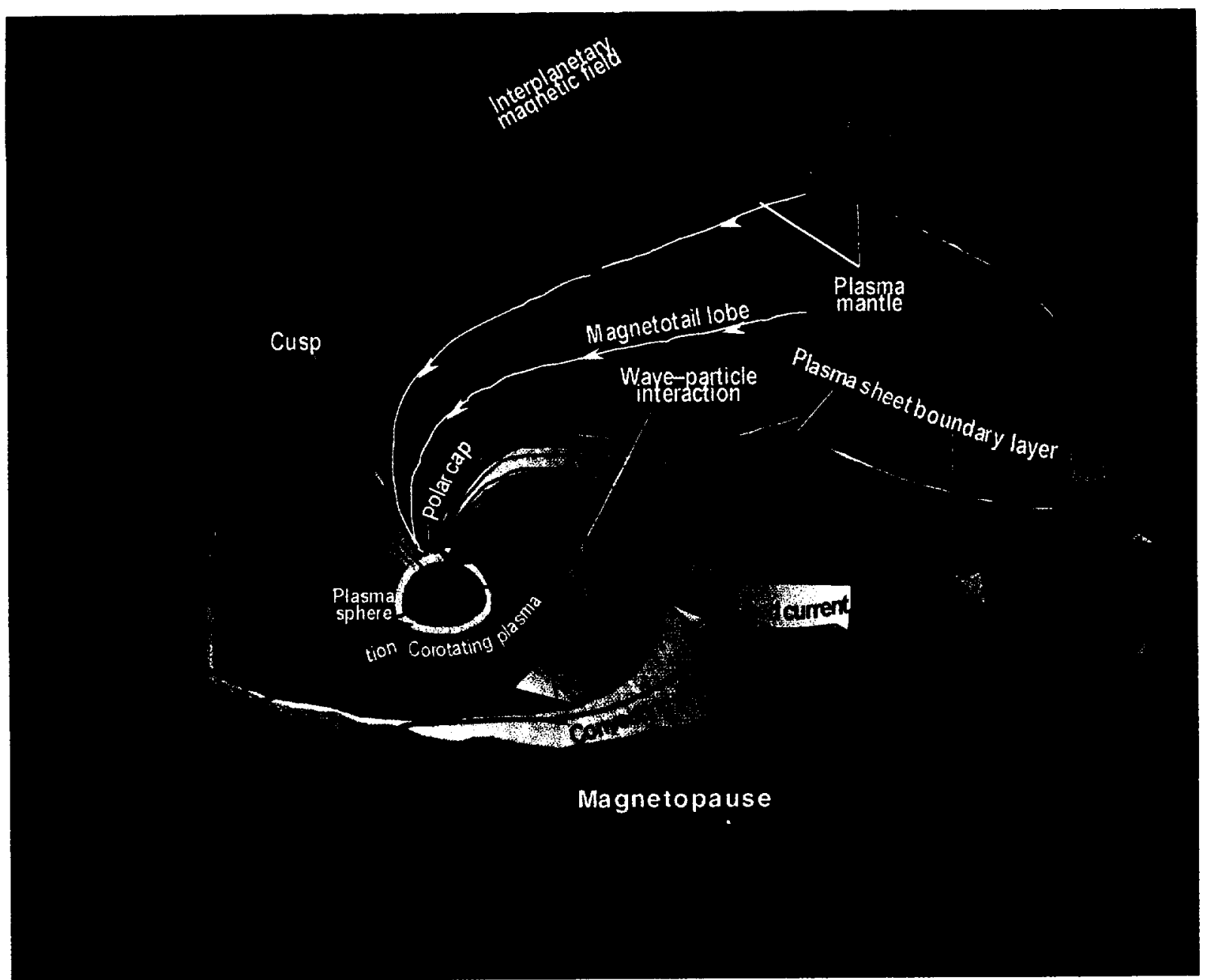

FIGURE 8 A diagram of the Earth's magnetosphere, showing the flow of the solar wind through the bow shock (where its flow goes from supersonic to subsonic) and around the current sheet known as the magnetopause, which forms the boundary between the magnetosphere and the solar wind. Through interconnection of magnetic fields and through entry of particles across the magnetopause, solar wind mass, momentum, and energy are transferred to the magnetosphere. Some of this energy ultinutely finds its way into the Earth's atmosphere. (Courtesy of Donald Mitchell. Applied Physics Laboratory, Johns Hopkins University.)

Freja) to get a near-global view of the polar atmosphere that functions as the sink for a significant fraction of the energy transferred from the solar wind to the magnetosphere.

Datal from several satellites (in particular, ISEE and AMPTE) have enabled identification of many of the important links in the transfer of energy, mass, and momentum from the solar wind and have highlighted the complex, nonsteady nature of the physical processes. Space physicists now know that transport of solar wind energy and momentum takes place across narrow boundary layers that separate regions with very different plasma conditions. The importance of these boundary layers in transport processes and particle acceleration in several regions of the magnetosphere, such as the plasma sheet, has also been identified. Phenomenological models of the global structure and dynamic processes developed from these satellite data are the basis of our present understanding of the magnetosphere.

The outer boundary of the magnetosphere (see Figure 8), called the magnetopause, is characterized by at number of transient phenomena and by two major boundary layers, one at low latitudes and one near the poles. Both of the boundary layers are important sources of plasma for the magnetosphere. but the mechanisms forming 
the boundary layers themselves are still not fully understood. Possible plasma entry mechanisms into the magnetosphere include diffusion and magnetic reconnection. Transient phenomena, such as Kelvin-Helmholtz waves, flux transfer events, and magnetic field perturbations due to small-scale pressure changes in the solar wind, also contribute to the entry of mass. momentum, and energy into the magnetosphere.

The steady merging of solar wind and magnetospheric magnetic fields, once thought to be the predominant entry process, does not seem to be sufficient to account for all of the features suggested by the data. The simple picture of steady-state merging has been replaced by one of transient. patchy, and small-scale merging with associated current systems that map to the polar cap and churn up the ionosphere. The evidence for reconnection at the magnetopause is very strong, although the microphysics of the reconnection process is still not understood. The interaction of the solar wind with the magnetosphere also sets up a convection system whereby plasma and "frozen-in" magnetic lield lines flow from the dayside of the magnetosphere to the tail and then return to the dayside. While the large-scale flow features have been confirmed by observations in the polar ionosphere. the specifics of the flow patterns throughout the magnetosphere are lacking. As would be expected when the input is transient, the convection is not steady. In fact, recent studies indicate that plasma flow in the magnetotail is usually bursty and irregular.

Two examples of dynamic processes that involve dramatic changes in the entire magnetosphere are magnetic storms and substorms. A magnetic storm is a period of enhanced geomagnetic activity, typically lasting many hours to days. During this period, particles are injected into the outer Van Allen belts to form an intense magnetospheric ring current that depresses the geomagnetic field at low latitudes. A portion of the ring current connects with the ionosphere, where it produces intense magnetic perturbations. Large magnetic storms can cause significant changes in the inner magnetosphere that are intimately associated with the lowest-latitude occurrences of the aurora. For example, recent observations by CRRES of the sudden formation of a "belt" of very energetic particles (tens of $\mathrm{MeV}$ ) show that large magnetic storms can cause major. long-lived changes deep in the inner magnetosphere. SAMPEX continues to study the creation of transient belts as well as their decay via precipitation into the atmosphere.

Magnetic storms and substorms are known to cause premature loss of communication satellites, disruption of radio communications, currents flowing in pipelines and cables. and interruption of electric power to consumers due to power-grid failures. Until recently. little attention was paid to magnetic storm events by the magnetospheric community, but observations from near-geosynchronous spacecraft such as CRRES now indicate that many aspects of magnetic storms are either poorly understood or not known. These include magnetic storm effects on the geomagnetic tail configuration, the temporal evolution in the processes of solar wind entry, and the energization of plasma from the ionosphere during storms.

In a substorm, the energy stored in the magnetic field of the tail is released explosively. The most frequently discussed substorm model has been magnetic reconnection in the region between 10 and 30 Earth radii downstream of the Earth. However. recent observations of substorms that are triggered on field lines at distances of 6 to 10 Earth radii seriously challenge this model and raise important theoretical questions. Moreover, the recent plasma observations at synchronous orbit show very different features from the familiar energetic-particle injections during substorms and are providing new views of the dynamics of energy transport into the inner magnetosphere. These observations are not fully understood. The types of physical processes that occur in the near-Earth magnetotail region during substorms remain one of the major unresolved issues of magnetospheric physics.

During substorms about $10 \%$ of the energy transferred from the solar wind into the magnetosphere is deposited in the auroral ionosphere. A significant fraction is deposited in the ring current and the midlatitude atmosphere, and some is also convected through the magnetopause into the magnetosheath. Limited studies of the deep tail made by ISEE-3 indicate that the rest of the energy flows down the tail and eventually back into the solar wind. Recent results from the Geotail mission are clarifying and expanding upon this initial picture. Because the structures in the distant tail can be complicated due to the tail's "memory" of the recent history of solar wind/ magnetosphere interactions and substorms, much work remains to be done to understand the physics of this important, yet largely unexplored, region.

The coupling process between the magnetosphere and the ionosphere is complex and highly variable. Largescale current systems, precipitating charged particles, and electric fields are the intermediaries that transport a significant portion of the energy and momentum resulting from the solar wind/magnetosphere interaction into the ionosphere. Precipitating particles from the magnetosphere are the major source of ionization in nightside 
midlatitude and polar regions. Those particles consist of keV ions and electrons in the auroral zone. lower-energy ions and electrons in the polar cap regions, and relativistic electrons and ions at tens to hundreds of keV at subauroral latitudes.

Currents and electric fields in the magnetosphere also play an important role in the magnetosphere-jonosphere (MI) coupling. The million-ampere magnetospheric currents flow into the ionosphere where they heat the thermal plasma and neutral gas. The ionization level governs the conductivity of the ionosphere and establishes the relationship between current and electric field. Magnetospheric energy is dissipated in the neutral atmospheric constituents in three ways: (1) heating due to particle precipitation. (2) heating from the dissipation of currents, and (3) energy and momentum transfer to the neutral molecules from collisions with ionospheric ions drifting in electric fields. The resulting changes can lead to feedback effects in the magnetosphere. For example, the largescale winds of neutral gas that persist even after the magnetospheric driver is turned off can drag ions across magnetic fields and may modify and even create new electric fields and current patterns in the magnetosphere. Another MI coupling effect comes from the flow of a large number of ionospheric ions up along the magnetic lield lines into the magnetosphere. These ions become energized by processes as yet unidentified and could play important roles in MI coupling and magnetic storm and substorm dynamics. Current understanding of these processes is based on many prior missions, including the Dynamics Explorer mission. Other MI coupling issues related to feedback of atmospheric and ionospheric effects into the magnetosphere are discussed in Chapter 4.

Not much is known about the transport processes in other planetary magnetospheres. The intense auroral emission observed from the jovian polar regions indicates robust MI coupling, but the identity of the precipitating particles (electrons, protons, oxygen or sulfur ions from lo) responsible for those emissions and the energy distribution of the particles have not yet been discovered. Researchers are even further away from knowing what acceleration mechanisms operate. The dependence of outer planetary aurora on solar wind coupling as opposed to internal dynamies, such as planctary rotation and interaction with satellites, is not yet established. The Cassini mission should help explain some of these unique interactions at Saturn; Figure 9 displays an artist "s conception of the saturnian magnetosphere based on the data from several previous flyby missions.

The following questions address both the flow of mass, momentum, and energy from the solar wind into and through the magnetosphere-ionosphere system and the impacts on the global structure and dynamics of this coupled system.

- How does the magnetic reconnection process operate under different boundary conditions? What role does reconnection play in the formation of the magnetopause boundary layers"?

- How does the solar wind plasma enter the magnetosphere? How important is reconnection compared to diffusion and direct entry processes?

- How does the magnetospheric convection respond to changes in the solar wind and interplanetary magnetic field? How is the magnetospheric convection affected by the highly variable ionization in the ionosphere and winds in the upper atmosphere?

- Where and how are substorms triggered and magnetic storms driven? What are the relative roles of ionospheric and solar wind plasma sources? How do small and mesoscale auroral features result from such global processes?

- How do the properties of both the near-Earth and the distant geomagnetic tail change during substorms and magnetic storms? Do local processes affect the global structure of the magnetosphere?

- What are the physical links between the component parts of the magnetosphere, shown in Figure 8 , such as the auroral electron precipitation regions, auroral upflowing ion regions, the plasmasphere, the ring current, boundary layers, and plasma sheet regions?

- What processes transport plasma between the ionosphere and the magnetosphere? Do the transport processes involve turbulent dynamics? Are electrons and ions transported by similar or different processes? How is ionospheric plasma energized in the magnetosphere?

- What are the global distributions of electric fields, current systems, and charged particles in the MI coupling region? How are magnetospheric and ionospheric electric fields and currents set up and how do they evolve over time?

- What is the accuracy of existing global models and simulations? What processes or structures do they fail to model? Does the magnetosphere have a ground, lowest-encrgy quasi-static state? If so, what is it? 


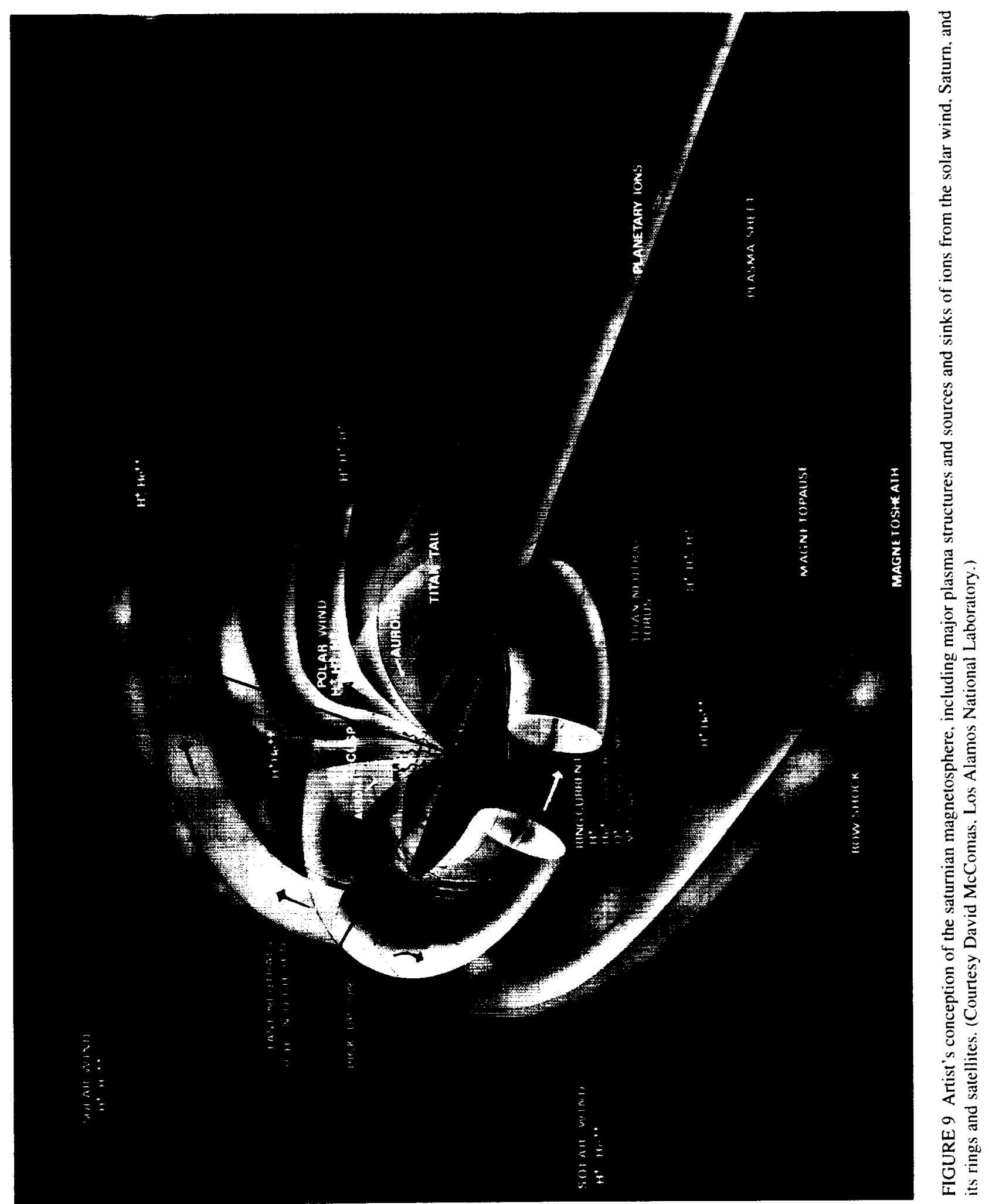




\section{CURRENT PROGRAM}

The International Solar-Terrestrial Physies (ISTP) program was specifically designed to study the flow of mass. energy, and momentum through the combined solar wind and magnetosphere systems. ISTP will employ an armada of spacecraft in the solar wind. magnetotail, and regions that cover the auroral zones and polar caps as shown in Figure 10. ISTP encompasses such spacecraft as NASA's Wind and Polar, the Japanese Geotail, and the European Cluster and SOHO. Additional contributions to ISTP will come from NASA s FAST and the Russian Interball-Aurora and Interball-Tail; from the older operating spacecraft. Akebono, Freja. IMP-8. DMSP. and GOES; and from the NOAA and Los Alamos geosynchronous satellites.

The Wind spacecralt will characterize the conditions in the solar wind. The Polar mission has the capability to continuously image the global auroral oval, and FAST will observe and characterize microphysical processes taking place in the auroral ionosphere. In addition. ISTP includes coordinated ground-halsed observations of ionospheric convection using radar, and mission-oriented theory teams to tackle the physics and model the processes. There will be special emphasis on determining the connection between the microphysics and the physics on global scales.

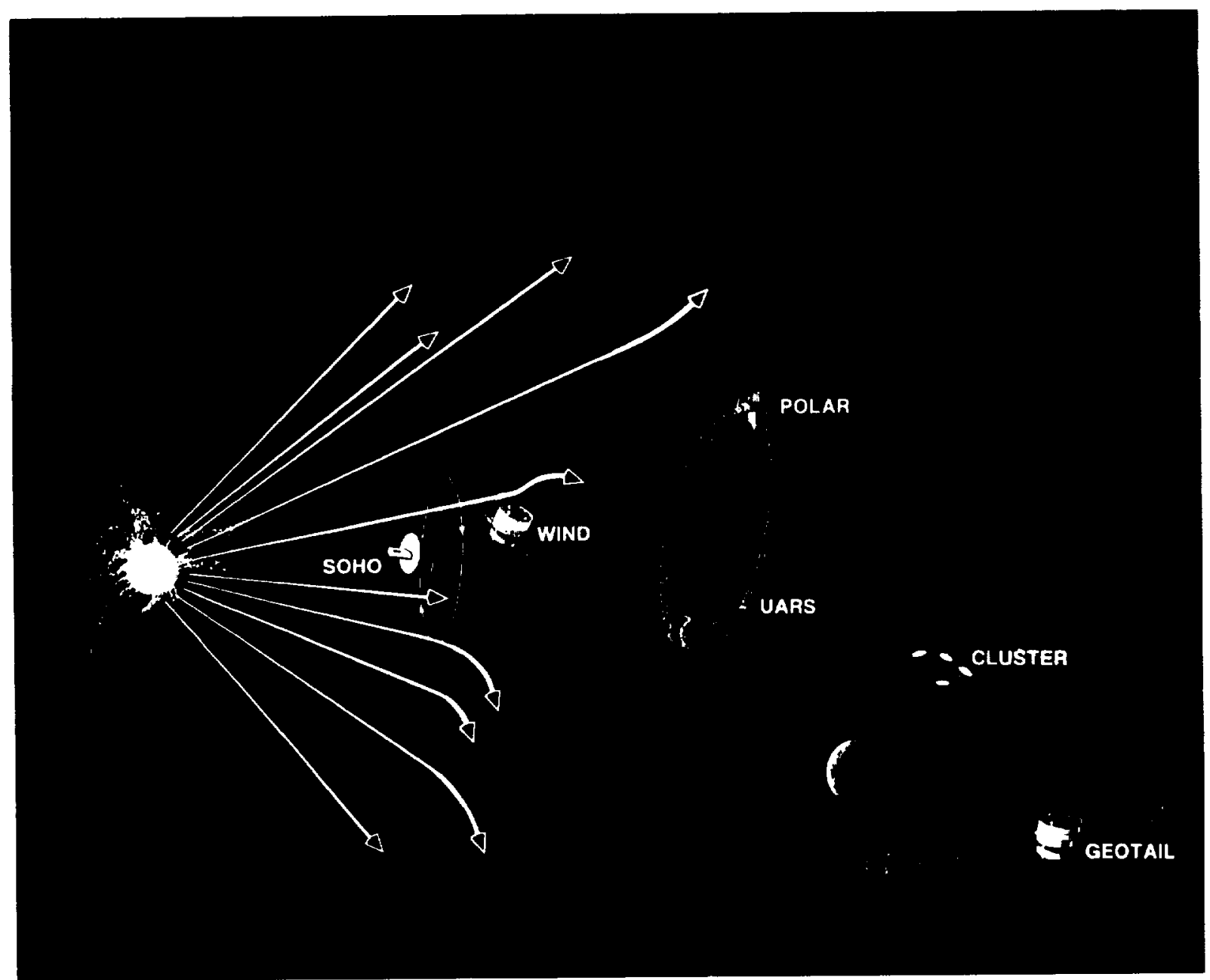

FIGURE 10 The locations of some elements of the International Solar-Terrestrial Physics program relative to major features of the Earth's magnetosphere (compare with Figure 8). Each spacecraft is designed to measure and enhance our understanding of successive links in that energy, mass, and momentum flow through the magnetospheric and atmospheric system. (Courtesy of Donald Mitchell, Applied Physics Laboratory, Johns Hopkins University.) 
Some of the ISTP spacecralt can change orbit to provide sequential coverage in more than one region. In particular, the Geotail spacecraft will be moved closer to Earth so that it hats an apogee near 30 Earth radii in order to examine reconnection processes in the near-tail region. It will be supported by Interball-Tail at 30 to 40 Earth radii and by spacecraft in geosynchronous orbit. By the time Geotail gets to the near-Earth tail, it is planned that Polar will be orbiting above the polar caps and will provide observations of auroral particles and fields as well as imalging. In addition, the FAST spacecraft to be launched in 1995 will provide in situ observations of the particles and fields alt low polar altitudes.

Geotail and Interball-Tail will provide observations of the low-latitude boundary layer on the flanks of the geomagnetic tail. Cluster and Polar will provide observations of the high-latitude boundary layer. On the dayside parts of its near-tail orbit. Geotail will observe the dayside low-latitude boundary layer and provide information on transient low-latitude phenomena at the dayside magnetopause. An extended Polar mission would also observe the dayside magnetopause. Taken logether, the set of Wind. Polar. Geotail, and Interball spacecraft, along with low-Earth-orbiting and synchronous-orbit spacecraft. will address the mechanisms involved in the global flow of matss. momentum. and energy across the boundaries of the system. The four co-orbiting Cluster spacecraft will enable researchers to observe the gradients associalted with the boundaries. This will provide observations of the boundary structures, how they evolve in time, and the processes operating there.

Concurrent with the ISTP program, the NSF"s CEDAR and GEM programs will use theory and available ground-based and satellite data to develop a global geospace environment model. GEM and CEDAR, along with NASA's Space Physics Theory program, will provide global models and simulations for investigating magnetospheric behavior. All will require stringent observational tests of their global predictions.

Several international and national organizations have established programs to increase the value returned from magnetospheric programs through the coordination of in situ observations, ground-based observations, data exchanges, and modeling and simulation studies. Among those efforts are the campaigns being organized by the Interagency Consultative Group and the international Solar-Terrestrial Energy Program.

Although the ISTP is potentially the most productive magnetospheric program ever mounted, there are some concerns. The ISTP concept will work only if the required simultaneous multipoint observations can be made. Also, the program has had many delays in key elements, and it is not clear whether the spacecraft will all fly in time to make the required simultaneous observations. In addition. several key observations will be missing. The regions originally chosen for detailed in situ sampling formed the minimum set required to infer large-scale features. but with the loss of the equatorial satellite (Equator), this minimum set no longer exists and there is a serious gap in the observing strategy. At this writing no replacement spacecraft is planned for this key region in the near-Earth equatorial tail between 8 and 15 Earth radii. This gap represents a double loss. It will not be possible to make observations in an important region where the substorm current disruption may be occurring in the tail. Adding to the science loss, the orbit that would have allowed a spacecraft to probe the near-Earth tail would also have cnabled the spacecraft to spend much of its time on the dayside near the magnetopause where it could observe transient events at the dayside magnetopause and in the low-latitude boundary layer.

The ISTP and FAST are also expected to make major contributions to our knowledge of MI coupling. The ISTP will add to our understanding of the magnetospheric input to MI coupling. of global electrical currents, and of the relation between global magnetospheric dynamics and conditions in the solar wind. FAST, passing through the low-altitude auroral acceleration region, will be able to address specific questions concerning what accelerates auroral particles. how parallel electric field and currents are established and regulated, and what specific instabilities and wave-particle interactions are involved.

The Earth's magnetosphere is not the only magnetospheric focus. Observations of the jovian aurora from the International Ultraviolet Explorer, the Hubble Space Telescope, and the Rosat X-ray satellite contribute to the study of MI coupling at Jupiter. The Galileo mission should also help clarify our understanding of the magnetospheric end of the jovian MI chain. Similarly, the Cassini mission to Saturn and its satellite Titan will yield new data on MI coupling for both Saturn and the interaction of Titan's ionosphere with the magnetosphere of Saturn (see Figure 9). In addition to in situ measurements of particles and fields, images of some parts of Saturn's magnetosphere will be obtained. Besides the optical imagers. the Cassini payload also contains an instrument that will provide images of the regions of the saturnian magnetosphere that emit energetic neutral atoms. Those data will in turn yield large-scale views of Silturn's magnetospheric energetic particle populations and their time variations. Although Cassini will not reach Saturn until 2004, it may obtain the first magnetospheric observations that are truly global in mature. 


\section{FUTURE DIRECTIONS}

By far the highest priority for understanding the processes that mediate the transport of mass, energy, and momentum through the magnetosphere-ionosphere system is the successful completion of the ISTP program. This includes ensuring that the planned satellite elements are launched in time for the required simultaneous measurements. The committees also strongly support efforts to recover the science lost when the original Equator satellite was canceled. It is also vital that the data analysis phase be sufficiently long and adequately funded, including support of scientists not currently on the instrument teams (i.e.. "guest investigators") and extending well beyond the end of the mission operations. The data obtained in this program will be extraordinarily rich and complex. and the lull scientific value will not be realized if any aspect-mission operations, the ground-based component, or datat analysis-is cut short.

Global imaging of the magnetosphere is the next-highest priority for magnetospheric investigations. Its importance has long been recognized and it should be the next observational thrust in the field. Current techniques developed for imaging and remotely sensing the magnetopause, the plasmasphere, and other plasma and energetic particle distributions and houndaries in the magnetosphere as well as in the aurora have the potential to provide important new insights about the workings of planetary magnelospheres as well as making this research more intelligible to the public. With the exception of auroral imaging. however, the technology has not received the flight opportunity it deserves. It is clear that remote sensing of the Earth's magnetosphere will provide information on its global dynamics and structure. It will also generate the data for refinement of inversion schemes and neutral geocoronal models needed to process the images. Figure 11 is the result of a simulation of magnetospheric images taken under storm-time conditions.

Detailed measurements are needed for MI coupling studies in the critical altitude regime from 5000 to $20,000 \mathrm{~km}$, where the bulk of the auroral acceleration occurs. Active experiments might be considered to investigate how natural processes work. For example, a combination of an electron gun and wave-particle instrument package for diagnostics launched on a rocket could provide detailed information on electric lield structures for accelerating auroral electrons along the geomagnetic field and might also shed light on causes of the complex and dynamic mesoscale structures that are observed in auroral ares. Other methods that hold promise for unraveling the details of the magnetospheric response to solar inputs center on the interpretation of ionospheric signatures of large- and small-scale currents, precipitation patterns, and plasma drifts. These ionospheric manifestations of important magnetospheric processes can be monitored and studied using strategically placed groundbased radars, magnetometers, and spectrometers. Coordinating these observations with spacecraft observations that supply information on the linkages of these ionospheric signatures, and the magnetospheric processes that generate them. can provide important global information on the properties of the magnetopause, magnetotail, and boundary layer. The relatively low cost and high scientific return of programs of this nature make their development and implementation a high scientific priority.

New knowledge about physical processes can also be obtained by studying magnetospheres where one or more of the boundary conditions are different from those at Earth. For example, the role of MI coupling in substorms should be examined at Mercury, which does not have an Earth-like ionosphere. In addition, the smaller spatial and shorter temporal scales of the hermean system are such that intrinsic time scales of processes such as tail instability and reconnection at the dayside can be examined more effectively, because the solar wind is more likely to remain constant for the duration of the event than is the case for the Earth's magnetosphere. Outer planets, such as Jupiter or Saturn, provide situations where plasma sources other than the ionosphere and solar wind are important and where energy sources other than the solar wind may dominate. In situ and global imaging observations of the magnetospheres of other planets will be important in furthering our understanding of space plasma processes. Radio techniques offer another ground-based diagnostic for monitoring radiation belts and thermal plasma properties; spaceborne lightning detectors are capable of making contributions related to auroral kilometric radiation as well as providing a method for remote sensing of auroral processes and atmospheric electricity on other planets.

Several regions of the Earth's magnetosphere are currently underexplored. For example, multispacecraft missions to skim the dayside magnetopause near the equator from dawn to dusk or from pole to pole would greatly enhance our understanding of the important physical processes contributing to the flow of energy, mass. and momentum from the solar wind into the magnetosphere on many different plasma scale lengths. Similarly, the auroral acceleration region (near altitudes of $\sim 1$ Earth radius) requires multispacecraft measurements with orbit 


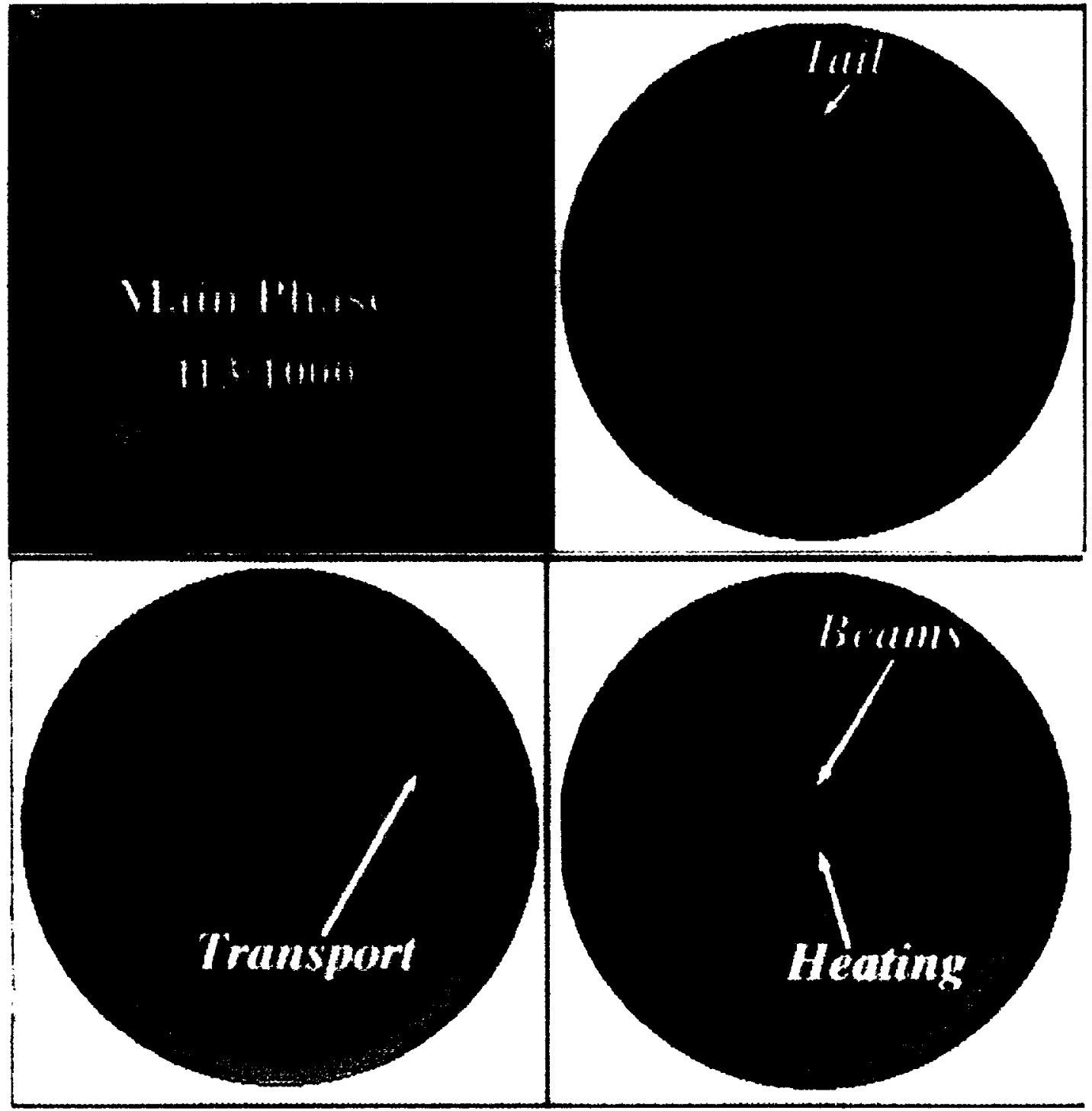

FIGURE 11 Simulations of images of the Earth's magnetosphere during the main phase of a magnetic storm, along with an auroral image taken during the main phase of the magnetic storm which provided the model input for the magnetospheric images. The auroral image was taken by the University of Iowa far-ultraviolet auroral imager on the Dynamics Explorer 1 spacecraft on April 22. 1988 (courtesy of L.A. Frank). Clockwise from upper right. the simulated images (which include a black circle indicating the position of Earth) are als follows: (1) An image of the plasmasphere taken in the emission of singly ionized helium at 304 angstroms. using a plasmaspheric model that includes a feature known as a plasma tail at the plasmaspause. The Sun is to the upper left of the box, resulting in reduced emission from the shadowed region behind the Earth. (2) The magnetosphere as imaged in low-energy neutral atoms. This simulation shows the emission of $\sim 5-\mathrm{keV}$ hydrogen atoms produced in charge exchange interactions between plasma protons and the cold hydrogen exosphere/geocorona. Signaltures of ion heating in the auroral zone and beams of up-flowing ions are indicated. (3) The magnetosphere as imaged in energetic neutral atoms. This simulation shows the enission of -30 -keV hydrogen produced by the same mechanisms as (2). The region labeled "transport" indicates a feature in the image produced by the dynamics of the drifting ion distribution in the Earth's ring current, a transient feature of the magnetic storm. (Simulation images courtesy of Edmond C. Roelof. John. Hopkins University. Applied Physics Laboratory.) 
maneuver capabilities, as does the near-Earth tail ( 8 to 12 Earth radii). These regions will not be addressed by the ISTP mission, especially not on the small scale where microphysics is addressed. Global inaging, in conjunction with in situ observations, leads to a more comprehensive view of any magnetosphere than multipoint satellite observations alone can provide.

All future magnetospheric observations would benefit from new technology development including design of smaller, lighter, more capable instruments and sophisticated data compression schemes. For example. new types of instruments are being developed that will provide enhanced global imaging of the magnetosphere. These include experiments to attempt radio sounding of magnetospheric boundaries. It is important that opportunitice for new instrument development and flight testing be available. 


\section{The Middle and Upper Atmospheres and Their Coupling to Regions Above and Below}

\section{SCIENTIFIC BACKGROUND}

Above the atmospheric region where "weather" takes place lies the middle and upper atmosphere, which provides a complex interface between the lower atmosphere and the space environment. Figure 12 gives a global view of the chemical, dynamical, and energetic processes that are thought to determine the properties of this transition region. Influences from above (solar radiation, energetic particles, magnetospheric electric fields and currents, meteors) and from below (trace gases, atmospheric waves. infrared radiation, thunderstorm electric fields) drive many interacting physical and chemical processes in the middle ind upper atmosphere, including global-scale winds, gravity waves and turbulence, photochemical reatetions. the global electrical circuit. and radiative, dynamical, and chemical transports of energy. The interplay between these inputs and the processes that redistribute energy from the individual source regions throughout the layer is not well understood. Figure 13 illustrates the different layers of this atmospheric region and some of the important processes that occur there. The middle atmosphere is defined as the region between the tropopause ( $101015 \mathrm{~km}$ altitude) and about $100 \mathrm{~km}$, while the upper atmosphere in the context of the present report extends roughly from $100 \mathrm{~km}$ to $1000 \mathrm{~km}$. The ions and free electrons that are present above $60 \mathrm{~km}$ at day and $100 \mathrm{~km}$ at night form the ionosphere. An important aspect of the magnetosphere-ionosphere-almosphere system is the presence of strong and interactive coupling processes that influence the time-evolution and dynamical properties of each element. This chapter considers those processes internal to the middle and upper atmospheres, as well as the influences of upper atmospheric processes on the nature of magnetosphere-ionosphere interactions. Magnetospheric influences on the ionospheric plasma and electrodynamic feedbacks to the magnetosphere are treated in Chapter 3.

In addition to contributing to understanding of how various physical and chemical processes operate and interact, studies of the middle and upper atmospheres have considerable practical value:

1. The middle atmosphere contains the ozone layer that shields the biosphere from solar ultraviolet radiation. Thus, changes in the amount of stratospheric ozone alter the biosphere's exposure to this harmful radiation.

Concern about stratospheric orone depletion has motivated a concerted international effort to understand and assess the problem and to take appropriate actions. It is now well established that the Antarctic ozone hole is caused by anthropogenic emissions of halocarbons. This conclusion, hased on ground-based measurements. four major airborne campaigns, and development and application of stratospheric models, together with increasingly strong evidence implicating halocarbons in global ozone depletion, underlies policy-including the 1990 London and 1992 Copenhagen amendments to the Montreal Protocol, and the U.S. Clean Air Act amendments-to accelerate the phaseout of halocarbons. Very recent measurements have shown that the rates of increase of chlorofluorocarbons (CFCs) and halons are beginning to decrease, while (FC substitutes are beginning to accu- 


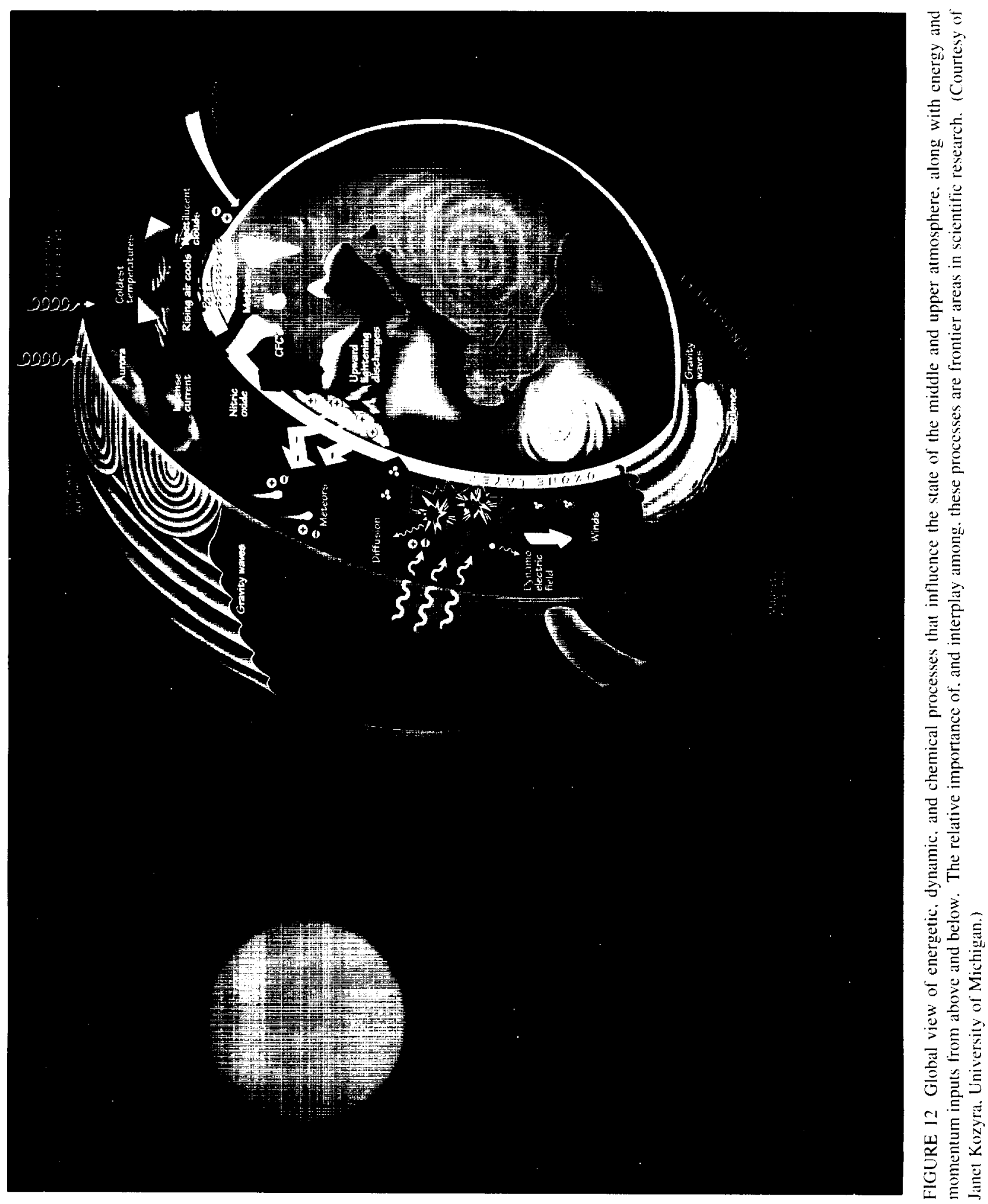



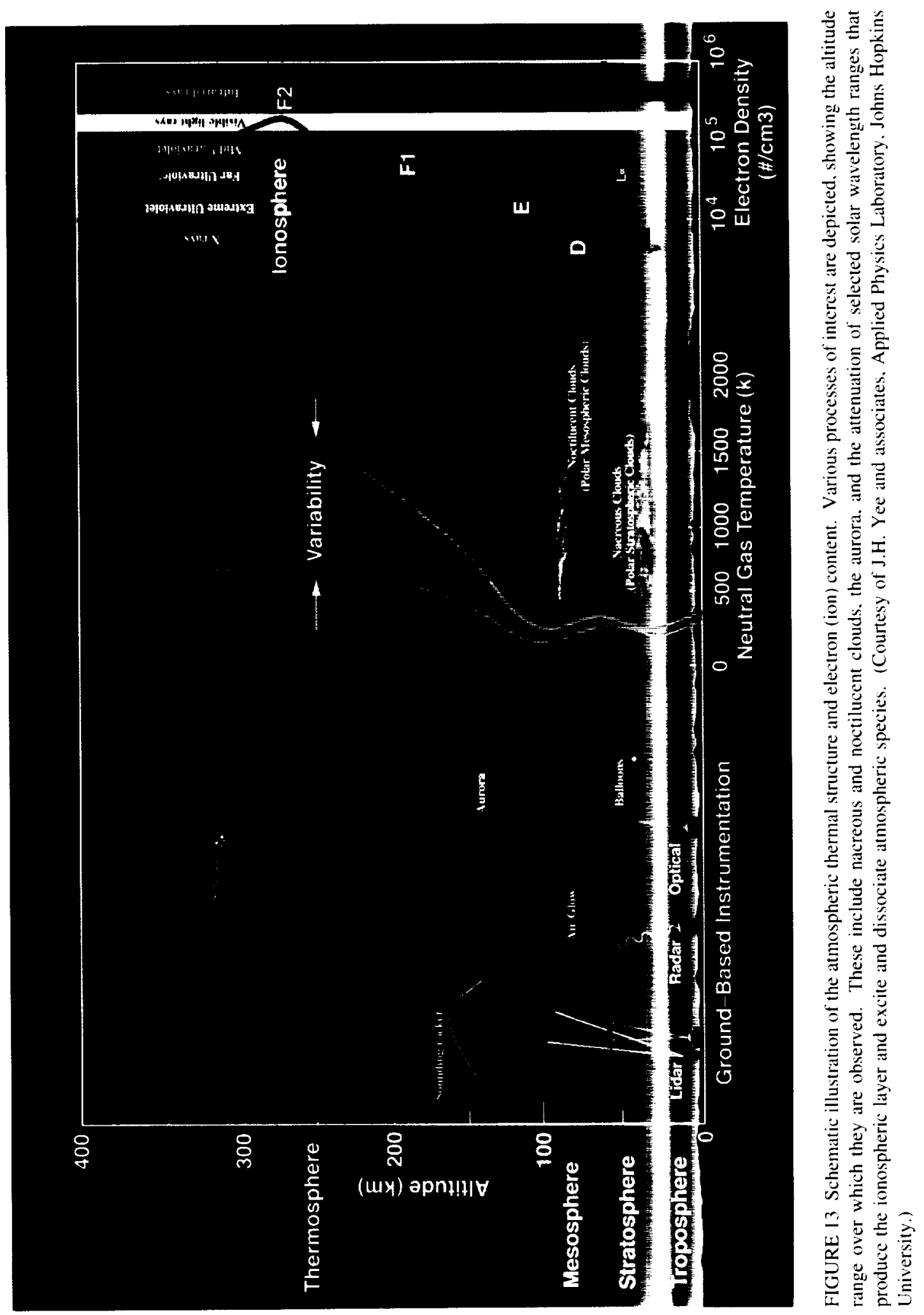
mulate in the atmosphere. These results, documented through a worldwide measurement network, verify that control of the use and emission of halocarbons is beginning to have an impact on atmospheric halogen levels.

Nevertheless, ozone depletion will likely worsen for at least another decade. Such depletion and the associated increase in ground-level ultraviolet radiation not only are significant environmental problems themselves, but are now known to be linked to climate change as well. Decision makers continue to turn to the world scientific community for advice.

How well can we forecast (hence manage) our ozone-layer's future? The following issues must be addressed by the scientific community to provide the needed input for public policy:

- What will be the global ozone losses and surface ultraviolet increases in the next 20 years, during which atmospheric halogen levels are expected to peak?

- What ozone losses and surface ultraviolet radiation increases could occur in the Arctic (and the Antarctic) during the same time period?

- What is the quantitative relationship between global, lower-stratospheric ozone depletion, radiative forcing of the Earth-atmosphere system, and climate change?

- What are the global climate impacts of the Antarctic ozone hole (which is expected to persevere well into the next century)!

- What are the most "ozone-friendly" halogen substitutes?

- How well do we understand the role of methyl bromide in ozone depletion?

- When will the ozone layer begin to be "rehabilitated"?

2. Changes in the middle atmosphere may affect the troposphere and influence the Earth 's climate. For instance, the CFCs are greenhouse gases. Thus, their increasing concentrations lead to climatic warming. The CFCs also lead to stratospheric ozone depletion. They may be responsible for observed ozone decreases in the lower stratosphere. Since lower-stratospheric ozone is itself a greenhouse gas, the combined radiative and ozone depletion effects of the CFCs lead to less greenhouse warming when their stratospheric ozone effects are considered.

3. Solar-driven disturbances in the upper atmosphere and ionosphere often affect space and terrestrial technological systems. leading to problems such as increased drag on satellites in low Earth orbit and disruption of radio communications.

These and other effects such as radiation and material damage, satellite charging. single-event upsets, and induced ground currents all fall into a class now called space weather phenomena. In general, space weather is the response of the near-Earth space environment to solar variability on short time scales. The Sun's energetic radiation and variations in its extended atmosphere, the solar wind, directly affect the ionization and heating of the Earth's upper atmosphere and the energy variability and content of the magnetosphere. This solar variability results in changes in the atmosphere-ionosphere-magnetosphere system that lead to far-ranging and often detrimental effects, such as loss of communication satellites and damage to power distribution systems. on technological systems that we have come to rely on.

The middle and upper atmospheres are strongly influenced by inputs of mass, momentum, and energy from above and below. The absorption of variable solar ultraviolet and $x$-radiation not only heats the atmosphere. but also initiates chains of photochemical reactions and ionizes the upper atmosphere to form the ionosphere. Additional ionization and chemical energy are provided by the precipitation of magnetospheric particles, galactic cosmic rays, and solar energetic particles, all of which respond to solar variability. Meteors produce ionized trails and are a source of metallic atoms and ions. Highly variable electric fields and currents of magnetospheric origin are a major source of energy and momentum to the high-latitude upper atmosphere, while tropospheric electrical storms can influence the electrical and chemical properties of the middle and upper atmosphere through upward conduction currents and discharges to the atmosphere. The electrical properties of the lower atmosphere in the region of tropospheric weather systems are strongly influenced by ionization due to cosmic ray impacts.

Intrared radiation from the Earth's surface and lower atmosphere help determine the energy budget of the middle atmosphere. Many of the middle atmosphere constituents are introduced from below, either directly, or indirectly in the form of chemical precursors. Volcanic eruptions increase the aerosol loading of the stratosphere. 
Gravity, planetary, and tidal waves that originate partly from the lower atmosphere grow in amplitude as they propagate upward, where they contribute to the momentum and energy budgets of the middle and upper atmosphere and produce turbulence that influences mixing processes.

There are important deficiencies in our knowledge of many of these inputs to the middle and upper atmosphere. The Sun's output of extreme ultraviolet and $x$-radiation is known to be highly variable. but only hard $x$ rays are regularly monitored. and the existing solar radiation measurements below $40 \mathrm{~nm}$ do not have calibrations adequate for many aeronomic applications. The accuracy of spectral irradiance measurements at longer wavelengths has improved in recent years, but the long-term variations are not well known. especially on the time scale of the solar cycle and longer. Average empirical models of particle precipitation from the magnetosphere and of high-latitude ionospheric electric fields and currents have been developed for a variety of geospace conditions, but space physicists often do not have complete knowledge of all key conditions in space coincident with a particular atmospheric event. Moreover, the distributions of particle precipitation. electric fields, and electric currents in the upper almosphere at any given time can be very different from the average conditions. The flow of electric currents in the global circuit above the tropopause and through the middle atmosphere and ionosphere is poorly understood. It is possible that present models of the global electric circuit, which emphasize quasi-DC current flow, have missed entirely one of the largest sources of electric charge movement illustrated by the recent observations of upward electrical discharges and gamma-ray bursts associated with thunderstorm activity. Figure I4 shows an impressive example of an upward electrical discharge from the top of a thundercloud to the ionosphere.

The sources of atmospheric waves entering the middle atmosphere from the troposphere are diverse and are not well quantified. except perhaps for atmospheric tides. The spectral characteristics, global distributions, and temporal variations of gravity waves and equatorial waves are poorly known.

Complex interactions among the energetics, chemistry, dynamics, and electrodynamics of the middle and upper atmospheres delermine their structure and variability. The absorption of solar ultraviolet radiation by ozone. and the absorption and emission of infrared radiation by carbon dioxide. ozone, water vapor, and other species, depend on the distributions of those components, which in turn depend on chemical processes and atmospheric circulation. In the upper mesosphere and lower thermosphere, heating and cooling are influenced by departures from conditions of local thermodynamic equilibrium and by exothermic chemical reactions. Heat conduction. viscous dissipation of sheared motions. and vertical air motions resulting from the effects of atmospheric waves also contribute to the heat budget. The net heating and cooling help determine the thermal structure of the atmosphere. which affects the emission of infrared radiation and the rates of chemical reactions. Theoretical studies predict that increasing anthropogenic carbon dioxide will lead to cooling of the upper atmosphere and significant reduction in high-altitude densities (Figure 15). Highly complex chains of chemical reactions can occur involving many different chemical compounds. Ozone in the middle atmosphere, and atomic oxygen in the thermosphere, are critical species that interact with many other compounds. The formation and the properties of aerosols depend strongly on atmospheric temperature and composition. while the presence of acrosols can significantly influence chemical and radiative processes, as well as plasma processes around the mesopause. The dynamics of the middle and upper atmospheres involve many types of interacting wave motions and the interaction of those waves with the mean circulation. For example. momentum transport by waves drives the tropical quasibiennial oscillation (QBO) and semiannual oscillation (SAO) in the middle atmosphere (Figure 16).

The maintenance and breakup of the winter polar vortex, of great importance to stratospheric ozone chemistry. is influenced by the strength of planetary waves. Small-scale wave processes in the mesosphere and lower thermosphere are believed to be the main source of turbulence at those heights. Turbulence contributes to atmospheric mixing and downward heat transport, and the dissipation of turbulence contributes to atmospheric heating. Tides are one of the main components of thermospheric dynamics. They display variability that appears to be related to their interactions with other wave motions in the middle atmosphere.

The magnetosphere is coupled to the middle and upper atmospheres through the ionosphere, and this coupling is highly variable in space and time. Flows of thermal and energetic plasma. large-scale current systems. and imposed electric fields provide the basic magnetospheric inputs to the ionosphere. These can significantly affect the altitudinal and horizontal distributions of plasma density, temperature, ionic composition, and conductivity. Strong electric fields are not confined to the polar regions. Occasionally, high-latitude electric fields can penetrate to subauroral latitudes. These fields can produce rapid ion drifts, plasma outllows, ion temperature increases, deep ionization troughs, and chemical changes in the ionosphere. The neutral atmosphere is also affected by energetic 


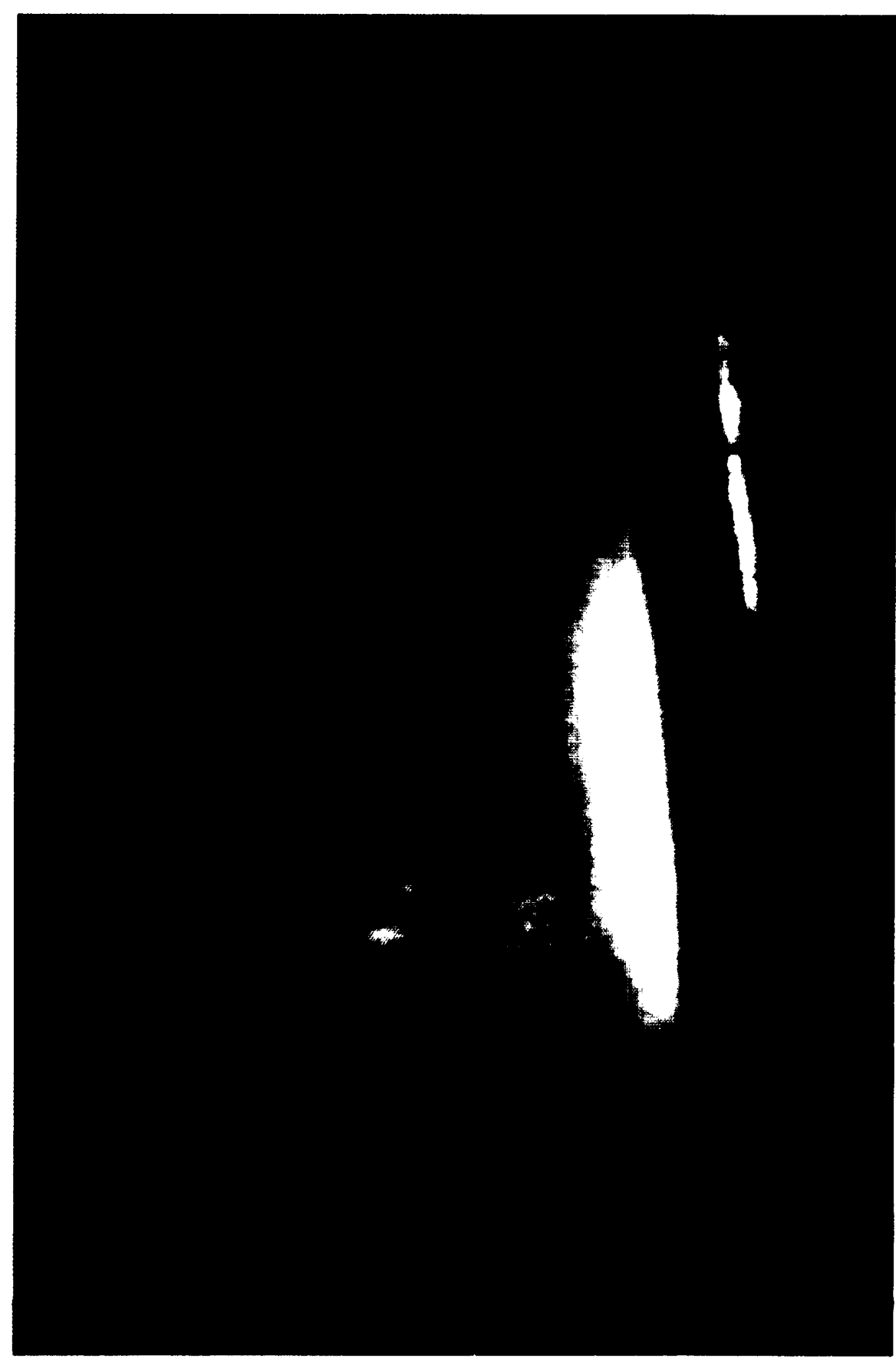

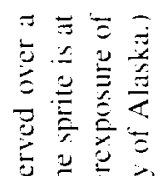

告

들

$\exists \cong$

$=\stackrel{2}{E}$

․ㅡㄹ

五势

事

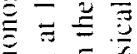

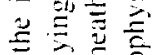

三

昰司

$-2=$

要

马

三丨

व

$\bar{\Xi} \underline{\Xi}$

을

言玄

主过

可

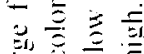

콣

可

要

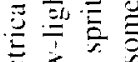

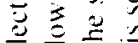

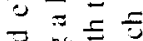

些哥

至

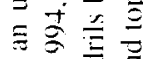

눌

을

挰

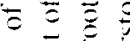

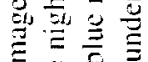

$\exists$ 늘

言焉焉

产

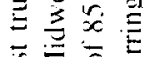

政

驱

$\bar{F} \Xi$

\pm 主

讨

品产

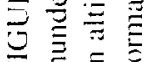



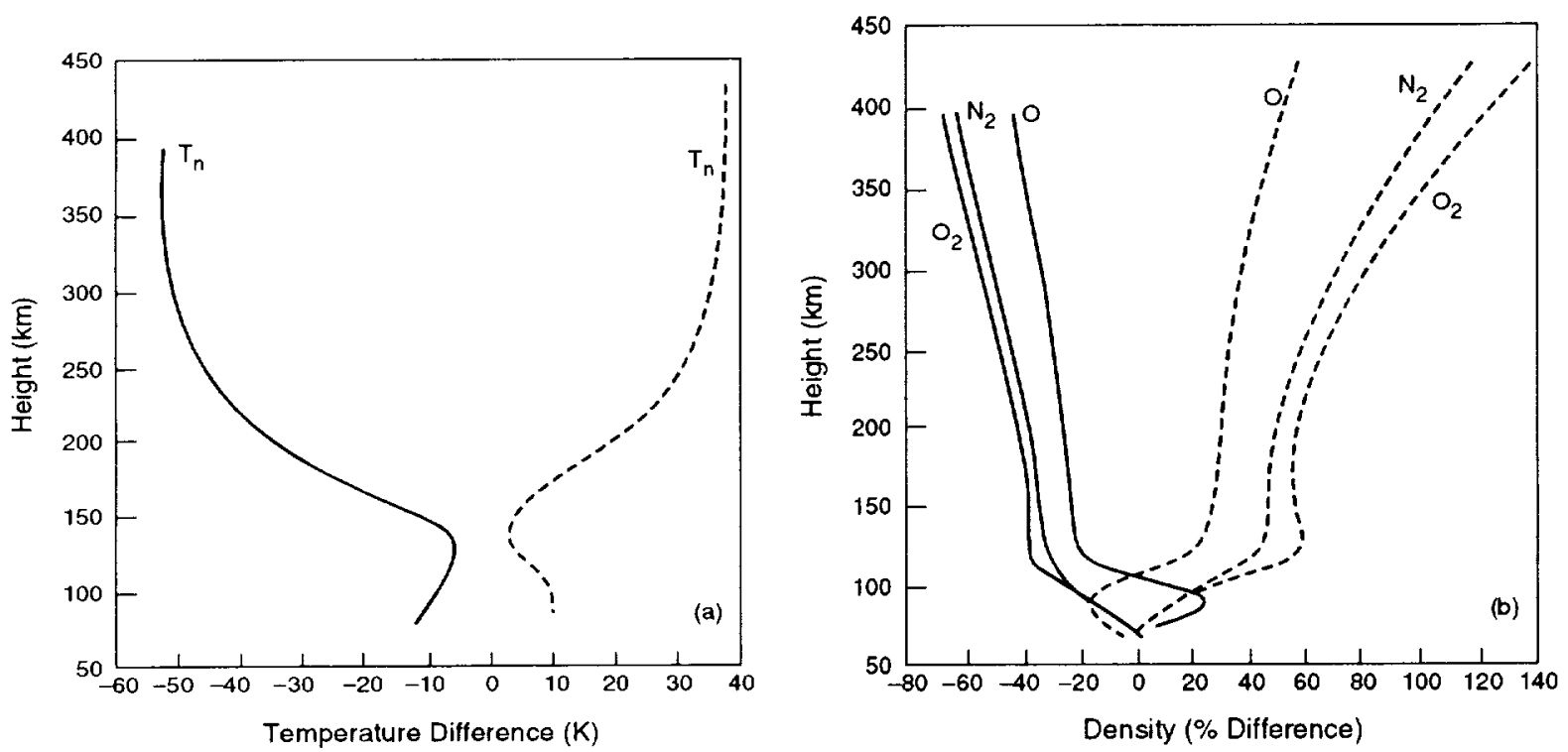

FIGURE 1.5 (a) Calculated globally averaged neutral gas temperature change. (b) Calculated globally averaged neutral gas density change of major thermospheric species from present day conditions for the calse where carbon dioxide and methane are doubled (solid line) and halved (dashed line). How will changes in carbon dioxide and methane modify the mean structure of the mesosphere and thermosphere? (Reprinted from R.G. Roble and R.E. Dickinson. Geophy's. Res. Lett. 16:1441-1444, 1989. Copyright 1989 by the American Geophysical Union.)

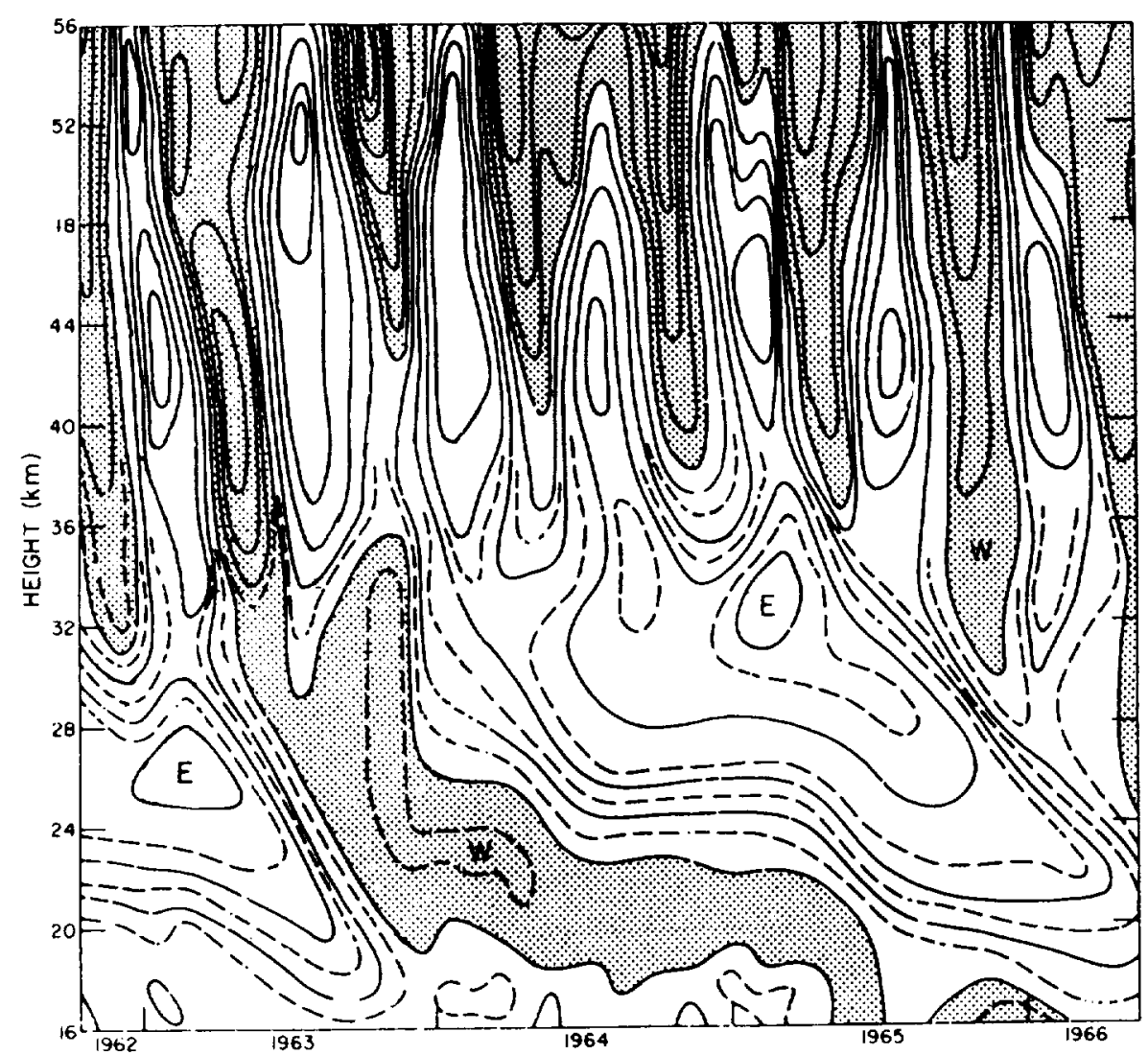

FIGURE 16 Time-height section of the mean zonal wind component near $9^{\circ}$ latitude with only the annual cycle removed. Shaded regions indicate winds from the west (w). Unshaded regions indicate winds from the east (e). Contours are drawn at $10 \mathrm{~m} \mathrm{~s}^{-1}$ intervals showing quasi-biennial oscillation below about $35 \mathrm{~km}$ and semiannual oscillation above. (After J.M. Wallace. Rev. Geophys Space Phys. 11:191, 222, 1973. Copyright 1973 by the American Geophysical Union.) 
inputs from the magnetosphere. Both chemical and dynamical changes lead to variations in the neutral density. The ionosphere carries the coupling current systems and provides feedback to the magnetosphere in the forms of ion outflow. conductivity changes, and dynamo electric fields. Tidal winds and propagating almospheric waves produce electrodynamic influences that are mapped from the lower atmosphere upward. These coupling processes result in a temporally and spatially structured system. For example. discrete auroral forms can range in size from a few ion gyroradii to global dimensions and can exist over time scales from milliseconds to hours.

There are many uncertainties in how all these processes operate and interact. An adequalte quintification of the roles of turbulence and gravity waves in heating and cooling these regions has not yet been achieved. The distribution of net atmospheric heating throughout the middle atmosphere is not well known. Our knowledge of the relevant chemical reactions and their kinetic rates is incomplete. For example, one area of active research addresses the problem of why currently accepted photochemical models predict too little orone in the upper stratosphere and mesosphere. There appears to be some "missing chemistry." Aerosol processes remain poorly understood, with respect to both their formation and evolution and their roles in heterogeneous chemistry. ritdiation. production of polar mesospheric clouds, and mesopause plasma irregularities that produce strong radar echoes.

Space physicists" understinding of the characteristics and effects of interactions among gravily waves, tides, planetary waves. and the mean circulation is far from complete. Momentum transport by gravity waves is believed to be critical for the mesospheric circulation, but observations of the relevant waves and their interactions with the mean flow are very sparse, while quantitative models of those effects are in their infancy. Full identification of the waves that help drive the QBO and the SAO has not yet been achieved.

The degree to which air inside the stratospheric winter polar vortices is exchanged with outside air is a subject of active debate. The manner in which gravity waves, tides, and planetary waves break, and the properties of turbulence thereby generated, are poorly understood. although some impressive modeling results have already been obtained (Figure 17). The quantitative effect of interactions between thermospheric winds and ionosphericmagnetospheric electric fields and currents on the structure and variability of the ionosphere and magnetosphere is similarly poorly understood. Researchers do not have useful predictive models of the complex system of generators, electrical charge storage systems, and both active and passive current paths that constitute the global circuit. Current research suggests that cosmic ray impacts in the lower atmosphere may produce paths of high electrical conductivity that result from cascading processes in electron production, but the effect of these spatially localized conductivity enhancements on the behavior of the global circuit is far from understood. The recent visual confirmation of the occurrence of lightning discharges upward to altitudes greater than $60 \mathrm{~km}$. coupling thunderstorm energy directly into the middle atmosphere, has dramatically underscored the paucity of knowledge in this area. These electrical discharges and other interesting observations of $x$-ray and gamma-ray bursts associated with active thunderstorm cells indicate that the direct upward electrical energy input into the ionosphere and thermosphere is much more important than previously thought. Furthermore, development of a better understanding of electrodynamical energy coupling through the middle atmosphere may also help clarify the role of atmospheric electricity in any possible effects of solar variability on the weather.

Many of the important physical and chemical processes that take place in the terrestrial upper atmosphere and ionosphere also occur in the environments of other planets and satellites. Other than Earth's, the upper atmosphere-ionosphere system that we have the most information about is that of Venus. This information came mostly from observations by the Pioneer Venus Orbiter. This unique but still skeletal database on the chemistry and energetics of the Venus atmosphere-ionosphere system has significantly advanced our understanding of those regions. There are some similarities between conditions at Venus and Earth, but also some very important and significant differences, mainly because of Venus's $\mathrm{CO}_{2}$-dominated atmosphere and the direct contact of the solar wind with that planet's ionosphere. Examples of important differences at Venus are the low and relatively solarcycle-independent thermospheric temperature and the ionospheric plasma temperatures that are significantly higher than those that would result from extreme-ultraviolet heating alone. The most glaring lack of data concerns the dynamics of the upper atmosphere of Venus. Space physicists have no direct information on the thermospheric winds. Terrestrial thermospheric general circulation models that have been modified to represent conditions at Venus predict large global wind systems, but there are no data to confirm those models.

The martian upper-atmosphere/ionosphere system is believed to be similar to that of Venus, but the possible presence of a small intrinsic magnetic field and a spin rate that is much faster than that of Venus may result in some potentially importint differences. Despite all the missions to Mars, the only direct aeronomical meatsurements 

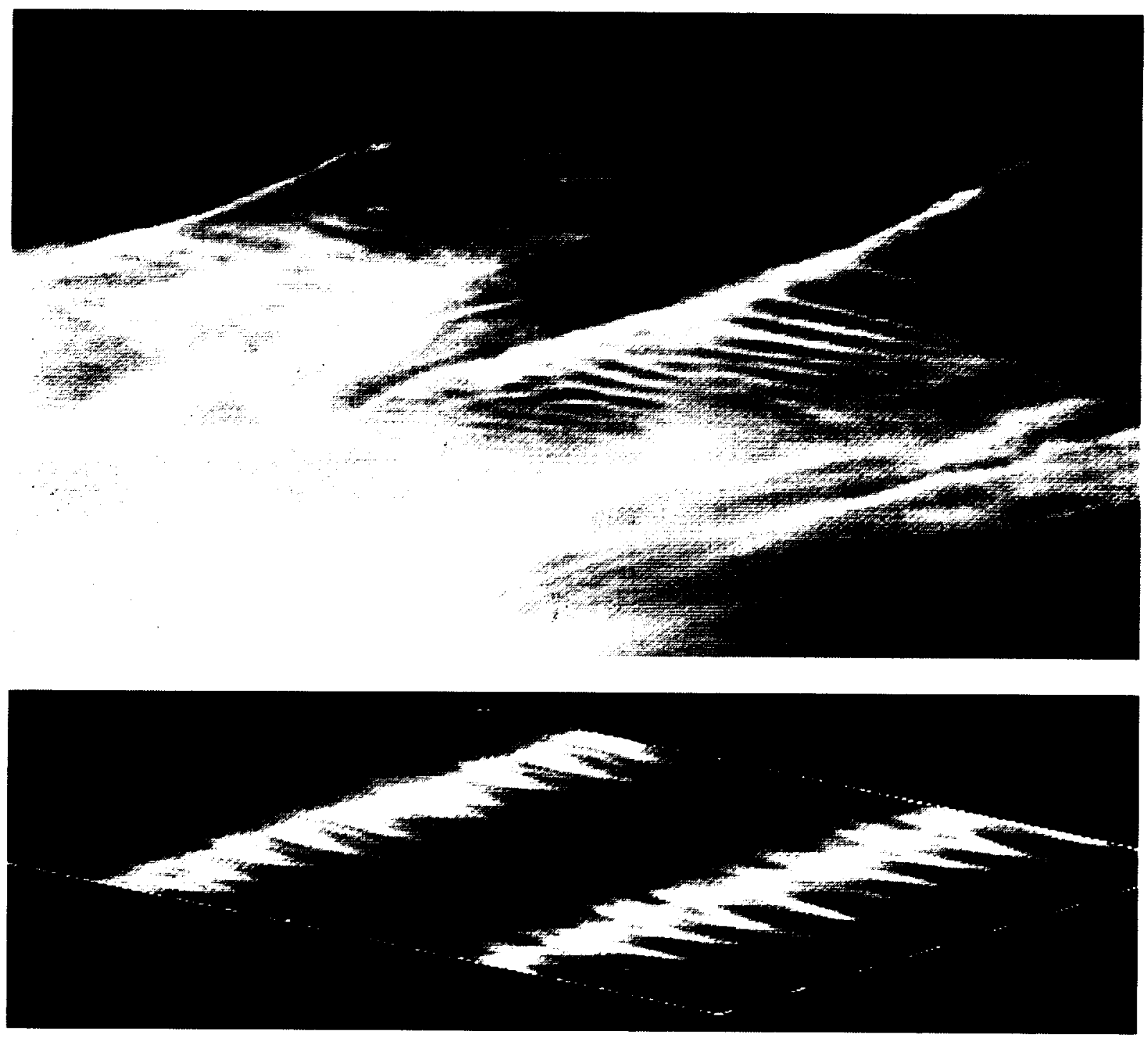

FIGURE 17 Top panel: A noctilucent (loud (NLC) display from Kustavi, Finlund 61 N. 21 E), on July 22, 1989, showing characteristic band and streak features. Botom panel: Simulated NLC brightness pattern from the three-dimensiontial gravity wave breaking model of D.C. Fritts. J.R. Isker. G. Thomas, and Ø. Andreasen. (jeophers. Res. Lett. 20(19):2039-2042. 19933. (Copyright 1993 by the American Geophysical Union.)

were those obtained by the neutral mass spectrometer and the retarding potential analyzer carried by the two Viking landers; the total databise is equivalent to that which would be obtained from a single rocket flight.

All the available information concerning the upper atmospheres and ionospheres of the outer planets and their moons come from the ultraviolet spectrometer and radio occultation observations obtained by the Pioneer and Voyager missions. This absolutely minimal database opened the door to at new and exciting world of mostly hydrogen-dominated atmospheres and ionospheres. Numerous theoretical models have been developed, but there are only weak observational constraints on these models. For example. in order to explain the relatively low electron densities observed in the ionosphere of Saturn, it has been proposed that a steady inflow of water from the ring plane has a significant impact on the atmospheric ion chemistry. changing the dominant ion species and thus the loss rates. This suggestion appears feasible but can be neither confirmed nor eliminated given the existing database. 
A major challenge to seientists studying the middle and upper atmospheres is to gain a comprehensive understanding of the relevant inputs and internal processes so that reliable models of these atmospheric regions can be developed that have useful operational and predictive capabilities. The following are some of the principal questions:

- What is the absolute solar spectral irradiance below $300 \mathrm{~nm}$. especially in the poorly determined region helow $40 \mathrm{~nm}$, and how does the irradiance vary on time scales of minutes to decades? How does the radiation vary as a function of altitude through the Earth's atmosphere?

- What are the spatial and temporal distributions of planetary waves, tides, gravity waves, and turbulence in the middle and upper atmospheres, and what are the sources of their variability? How do they affect atmospheric structure, and which wave motions drive the QBO and the SAO?

- What causes the apparent coupling between the QBO and extratropical dynamics?

- What are the global distributions of electrodynamic energy and momentum inputs to the middle and upper atmosphere, viewed as a time sequence rather than in a highly averaged or climatological sense? What corresponding electrical, chemical, and dynamical changes do these inputs force in the neutral atmosphere on comparable time scales? How do the coupled neutral and ionized components of the atmosphere evolve as a disturbance proceeds"? How do long-lasting and large-scale changes in the coupled system manifest themselves in altered global patterns of conductivity, joule heating, ion convection velocities, and current systems and, ultimately, feed back on and alter the interaction with the magnetosphere?

- What is the importance of lightning (including all forms of transient discharge) to middle-atmospheric chemistry and clynamics and to ionospheric electrical phenomena? What are the generators, electric charge storage systems. and active and passive current paths that constitute the middle and upper atmospheric portions of the global electric circuit?

- How do coupled radiative, chemical, electrical, and dynamical effects determine middle and upper atmospheric structure and variability?

- What are the temporal and spatial distributions of aerosols in the stratosphere and mesosphere? What physical processes control their distribution, and what are their radiative and chemical effects?

- What are the contributions to climatological changes in the middle and upper atmosphere from solar influences. volcanic eruptions, anthropogenic effects. and changes in the lower atmosphere?

- What are the dominant acronomic processes in the upper atmospheres and ionospheres of other planets or satellites? How do they differ from terrestrial processes, and why? Can models of terrestrial processes be improved or constrained by data obtained at other planets?

\section{CURRENT PROGRAM}

The first comprehensive global observations of temperature, winds, and some minor chemical species in the stratosphere and into the mesosphere are currently being made by the Upper Atmosphere Research Satellite (UARS) launched in September 1991. The Midcourse Space Experiment (MSX) spacecraft, scheduled for launch in 1995. will add further valuable data on the composition and temperature of the middle and upper atmospheres, but only for a limited number of event-oriented campaigns. Solar ultraviolet radiation and energetic particles are also being measured by UARS and SAMPEX as well as by polar-orbiting NOAA and DMSP satellites. Periodic shuttle missions are also being flown that make middle and upper atmosphere measurements. Figure 18 shows some early UARS results illustrating that significant effects on stratospheric composition may result from downward transport from the mesosphere.

NSF's CEDAR program, together with other ground-based observations (radar, lidar. spectrometers, interferometers, imagers, and photometers), continues to provide information on winds, chemical composition, temperature, radiative emissions, and ionospheric densities and drifts in fixed geographic locations, on scales and in locations not accessible to satellite observation. Much of the current knowledge about Antarctic ozone depletion has been obtained from intensive ground-based remote sensing, balloon. and aircraft campaigns. Similarly, current knowledge of gravity wave fluxes. mesospheric tides and planetary waves, the temperature structure of the summer mesopause, and the structure and variability of the ionosphere and thermosphere is being advanced by application of ground-based techniques.

Suborbital experiments are a key component of the current program. For example, rockets and balloons can 


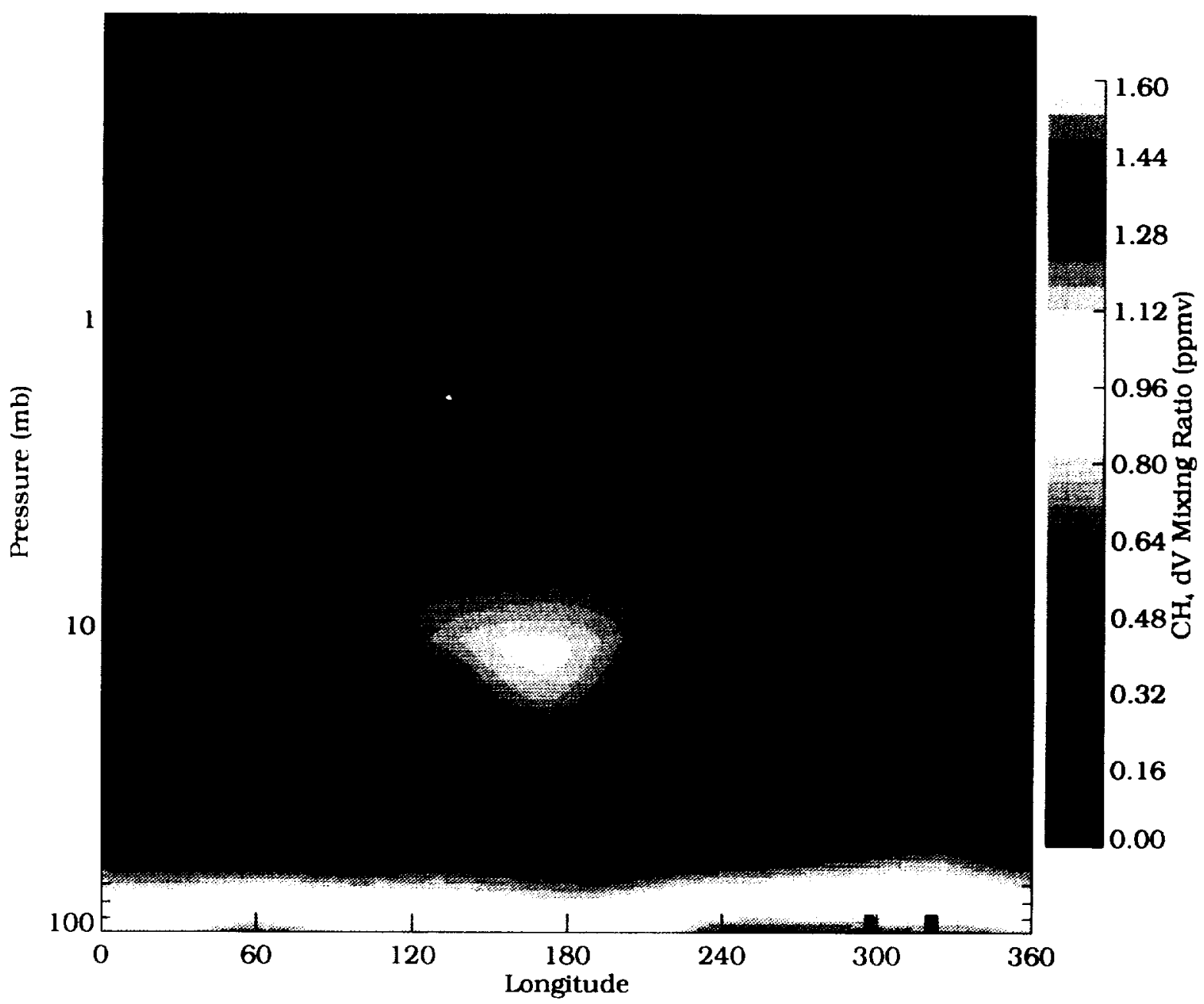

FIGURE 18 Two recent measurements from the Halogen Occultation Experiment (HALOE) on UARS. Above: HALOE measurements of methane $\left(\mathrm{CH}_{4}\right)$ as a function of longitude and pressure altitude at $76^{\circ} \mathrm{S}$ on October 22, 1991. Note the signature of descent (blue region at a longitude of approximately $300^{\circ}$ ) from the mesosphere (above $1 \mathrm{mb}$ ) to the lower stratosphere (about $30 \mathrm{mb}$ ). Facing page: HALOE measurements of reactive nitrogen oxides $\left(\mathrm{NO}+\mathrm{NO}_{2}\right.$ ) as a function of latitude and altitude for the period from July 11 to August 30, 1992. Note the signature of descent from the thermospheric source of NO at high southern latitudes (yellow and green regions on left-hand side) and its connection to the stratospheric distribution of reactive nitrogen oxides (yellow regions across center of image). (Courtesy of J.M. Russell III, NASA/Langley.)

measure neutral and ion composition, turbulence, electric and magnetic fields, and energetic particles and can obtain altitude profiles of physical properties to determine, for example, the vertical structure of auroral features. A series of polar-orbiting satellites (DMSP, HILAT, Polar BEAR, Viking, Freja, Akebono) have obtained largescale images that define the global morphology of the aurora and measure the energy spectra of particles precipitating from the magnetosphere. ISTP will add to our understanding of the magnetospheric input to the upper atmosphere by placing in situ measurements of precipitating particles and fields in the context of the global magnetospheric configuration. Freja is making high-time-resolution measurements of auroral zone particles and fields, providing a key link in our understanding of the magnetosphere-ionosphere-atmosphere coupling. FAST will extend the measurements of electrodynamic parameters and energetic particles to higher resolution in three dimensions.

Research into the role of the middle and upper atmospheres in global change requires well-integrated efforts in long-term and well-calibrated monitoring of selected variables in the climate system. Some of these long-term 


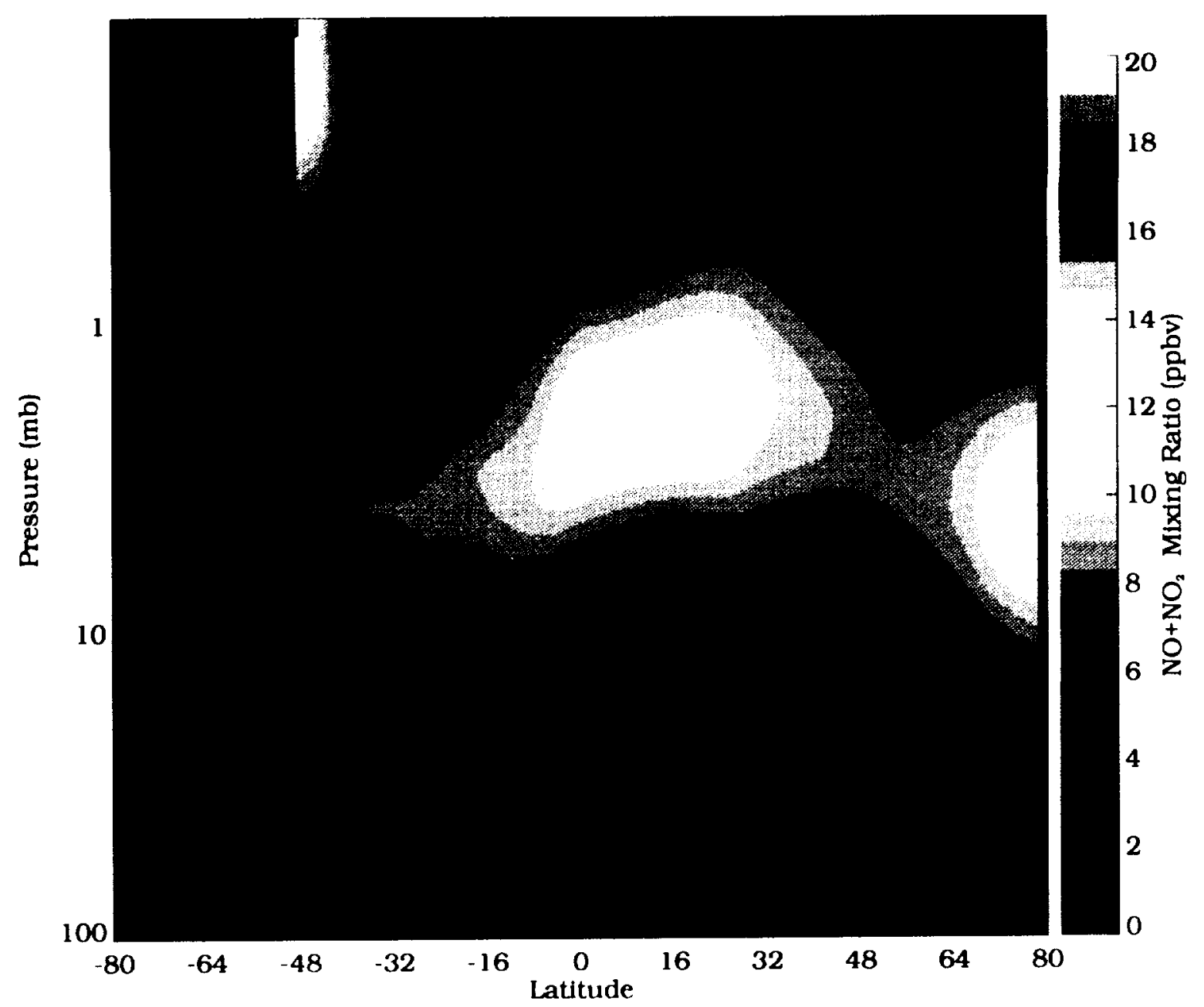

monitoring programs currently exist for the stratosphere. Total ozone has been monitored from the ground-based Dobson network for almost 40 years, and there have been continuous measurements of total ozone from space for over 15 years by NIMBUS 7, TIROS, and Russia's Meteor-3 spacecraft. New algorithms are being developed that might allow consistent, well-calibrated ozone profiles to be recovered from the NIMBUS and TIROS data, but those measurements will not give ozone profile information in the lower stratosphere. The SAGE instrument has given ozone profile measurements in this region, and the ILAS instrument on the Japanese ADEOS spacecraft should give similar information. Long-term measurements of ultraviolet-B radiation at the Earth's surface have not been sufficient to give reliable trends related to changes in stratospheric ozone because of inadequate calibration and spectral resolution as well as lack of simultaneous measurements of important accompanying atmospheric variations such as clouds and aerosols.

Temperature profiles that extend up into the lower stratosphere are being measured by the worldwide radiosonde system, but there are some problems obtaining a long-term, well-calibrated temperature record from the radiosonde network because it is limited to land-based launch facilities and there have been changes in instrumentation, procedures, time of launch, and station locations. The situation is much worse for satellite measurements of stratospheric temperatures because changes in satellite instrumentation and in the retrieval algorithm make it necessary to calibrate these data against independent measurements, which is no longer possible because of the declining number of rocketsonde launches. Future plans involve calibration against lidar temperature measurements, but that system has not yet been implemented. Thus, researchers are left with an unsatisfactory monitoring system for stratospheric temperatures.

U.S. agencies have been working with international organizations to monitor a number of stratospheric 
variables through the Network for Detection of Stratospheric Change (NDSC). The aim of the network is to obtain a continuous, well-calibrated data record for the stratosphere that can be used to calibrate satellite measurements. The measurements will be very valuable, but they will exist at a very limited number of stations around the world. The World Climate Research Program has recently implemented a program on stratospheric processes and their role in climate to address some of the issues of the middle atmosphere and climate.

The centerpiece of the space-based Earth observation program is the EOS mission, which was originally planned to provide comprehensive, well-calibrated measurements over a 1.5-year period. Budget problems have led to its substantial descoping. so that now it does not include measurements of particles and fields, and its observations of the middle atmosphere are limited to several key measurements of stratospheric composition and temperature. These EOS middle atmosphere measurements are not anticipated to begin for another 8 to 10 years. Thus, only the NOAA operational and other climate-monitoring systems such as NDSC can be relied on for longterm U.S. measurements. Other ground-based programs, such as NSF's CEDAR program, have very useful capabilities for those purposes, but that program is not configured for long-term measurements. Measurements from the Japanese ADEOS and the European Envisat platform may also make important contributions.

Computer models have been developed for studying the coupled dynamics, energetics, and chemistry of the middle and upper atmospheres. The models provide a valuable beginning to the study of the fully coupled problem, but they all have limitations with regard to the level of detail included, the coupling with lower and/or upper levels of the atmosphere, the number of spatial dimensions, and/or the computational time required.

The most ambitious planetary aeronomy observations in the current program are those planned for Saturn's satellite Titan as part of the Cassini mission. The European-provided Huygens probe will obtain atmospheric profiles from the middle atmosphere down to the surface, while the main Cassini spacecraft will carry a mass spectrometer for the analysis of ions and neutral species each time the spacecraft dips through the upper atmosphere for orbit-changing gravity assists. A magnetometer is also expected to be placed in orbit about Mars as part of the Mars Surveyor program.

\section{FUTURE DIRECTIONS}

In the atmospheric sciences, the application of the scientific method usually proceeds by continual interaction between theory and observations in the following manner. Highly simplified models, having few degrees of freedom, are advanced to explain specific observations. Typically, there are areas of disagreement between theory and observations that motivate improvements in the theory and the need for more observations. The mechanisms contained in these simplified models are then imbedded in more comprehensive models that contain a variety of interacting physical processes, and analyses of the results from those models are then compared to similar analyses of atmospheric observations. Thus, all through this process, it is the mismatch between theory and observations that motivates the need for more observations and the formulation of improved models. It is this process that motivates researchers' future needs.

Global observations of the temperature, winds, composition (including the characterization of the physical and chemical properties of aerosols). and radiative emissions should be oblained and continually compared against models in order to understand middle and upper atmosphere processes and the coupling to regions above and below. It is important that the UARS satellite program (which is making important advances in stratospheric observations, and some inroads into the mesosphere) and the CEDAR ground-based program maximize the scientific return from the sizable investments already made. Furthermore, our current understanding of the electrodynamics of mesoscale and small-scale structures is poor because of the lack of high-resolution. magnetically conjugate measurements in the ionosphere, and because of the lack of detailed measurements of electrical. chemical, and optical phenomena above thunderclouds in the middle atmosphere. The latter observations might also provide a key for understanding the origin of atmospheric gamma-ray bursts. As discussed above, the feedback processes involving the magnetosphere and ionosphere are temporally and spatially scale-size dependent. The coordination of high-resolution FAST in situ measurements with ground-based and rocket observations could contribute to assessing the effectiveness of the feedback processes as a function of temporal and spatial scale size. Future programs will build on the results of these current programs.

There are good prospects for developments in lidar techniques to enable high-resolution, daytime and nighttime measurements of middle-atmospheric winds and temperatures, as well as the concentrations of certain minor constituents. Scanning ground-based and airborne lidars enable measurement of the horizontal as well as vertical 
structures of atmospheric features. The global network of relatively inexpensive wind-measurement radars should be expanded, and the data should be used jointly with satellite observations to allow clear definition of the longitudinal and latitudinal variations of mesospheric tides, planetary waves, and gravity waves. These important waves not only give rise to wind oscillations like the QBO and SAO, but also give rise to stratospheric warming and determine the nature of the winter polar vortices. Passive optical and microwave techniques for middleatmospheric observations also show great promise for contributing to better understanding of minor constituents like ozone and water vapor.

For understanding the complex behavior of the mesosphere/lower-thermosphere region, atmospheric measurements must be accompanied by well-calibrated measurements of solar ultraviolet, extreme ultraviolet and soft $x$-radiation, energetic particles, and electrodynamic parameters. Atmospheric wave processes including tides and gravity waves and their interactions are particularly important for this region. Our understanding of the lower thermosphere and mesosphere is currently based on an observational database that consists of ground-based and rocket-borne observations as well as campaign-oriented measurements from the space shuttle. These sources of data, though extremely useful for improving our understanding of this region, are restricted to fixed geographic locations and thus are insufficient to allow us to characterize the complex global behavior that involves distributed energy sources along with large-scale dynamics and both horizontal and vertical transport of energy. UARS has recently expanded this database by providing global observations of winds, temperatures, and some minor constituents over altitudes extending into the mesosphere. Even with the global characterization of a limited number of parameters through the UARS mission, fundamental questions still exist about basic parameters and their variability, and about important radiative, chemical, dynamical, and electrodynamical phenomena. One way to address the need for global data is through a spacecraft mission that uses remote sensing techniques to sample the required altitude range. However, a single orbiting spacecraft has a limited ability to resolve tidal variations necessary to understand the mean state as well as variations about the mean state. For example, UARS. given its orbital inclination and altitude, takes about $\mathbf{3 6}$ days to make measurements over all local times if both daytime and nighttime measurements are included. If better resolution of phenomena with large local time variations (tides, for example) is desired, it is necessary to use multiple spacecraft or to supplement the space observations with groundbased observations that have good local time resolution. CEDAR observations are particularly valuable for those purposes. The understanding of the mesosphere/lower-thermosphere region requires coordinated measurement of atmospheric dynamics, especially wave motions, and energy inputs from space.

The study of climatological changes in the middle and upper atmosphere requires well-calibrated satellite and ground-based monitoring of the composition, dynamics, and temperature and of the inputs to this atmospheric region from above and below. The measurements need to extend at least over one solar cycle in order to adequately separate solar-induced variations from anthropogenic variations. Further development of models of these regions is needed not only to understand the complex workings of these regions but also to predict their future changes. Ideally, these models should be three-dimensional, should include realistic photochemistry and radiation, and should have the capability of fully resolving phenomena with very disparate scale sizes and of representing nonlinear coupling processes that combine to determine the behavior of the global system. Work with such models has proceeded slowly. One reason is that their computational requirements often exceed available resources. Global three-dimensional models must keep pace with instrument measurement capabilities, take advantage of new hardware architectures and software developments (e.g., parallel processing, high-performance computing), and be an integral part of future plans for measurement programs.

Although there is much ongoing research on middle and upper atmosphere problems in ozone research (within NASA and NOAA, for example) and on ground-based aeronomic measurements and their interpretation (within NSF), there has been inadequate contact between these two communities. Research on the coupled middle-upper atmosphere system could be accelerated by the establishment of a coordinated research program that takes a more holistic view of research in this area.

Understanding the time-dependent, dynamical coupling processes within the global electrodynamic system requires large-scale maps of key energy and momentum inputs, as well as ionospheric responses, with significantly better spatial and temporal resolution than has been available in the past. Past work has relied on broadly averaged parameter fields. The capability already exists to invert large-scale photometric images of the auroral regions into maps of estimated ionospheric conductivity and estimated energy inputs from particle precipitation. Improvements in imaging detector technology and electronics have enabled the development of a new generation of ground- and space-based optical instruments with greatly increased sensitivity and associated improvements in 

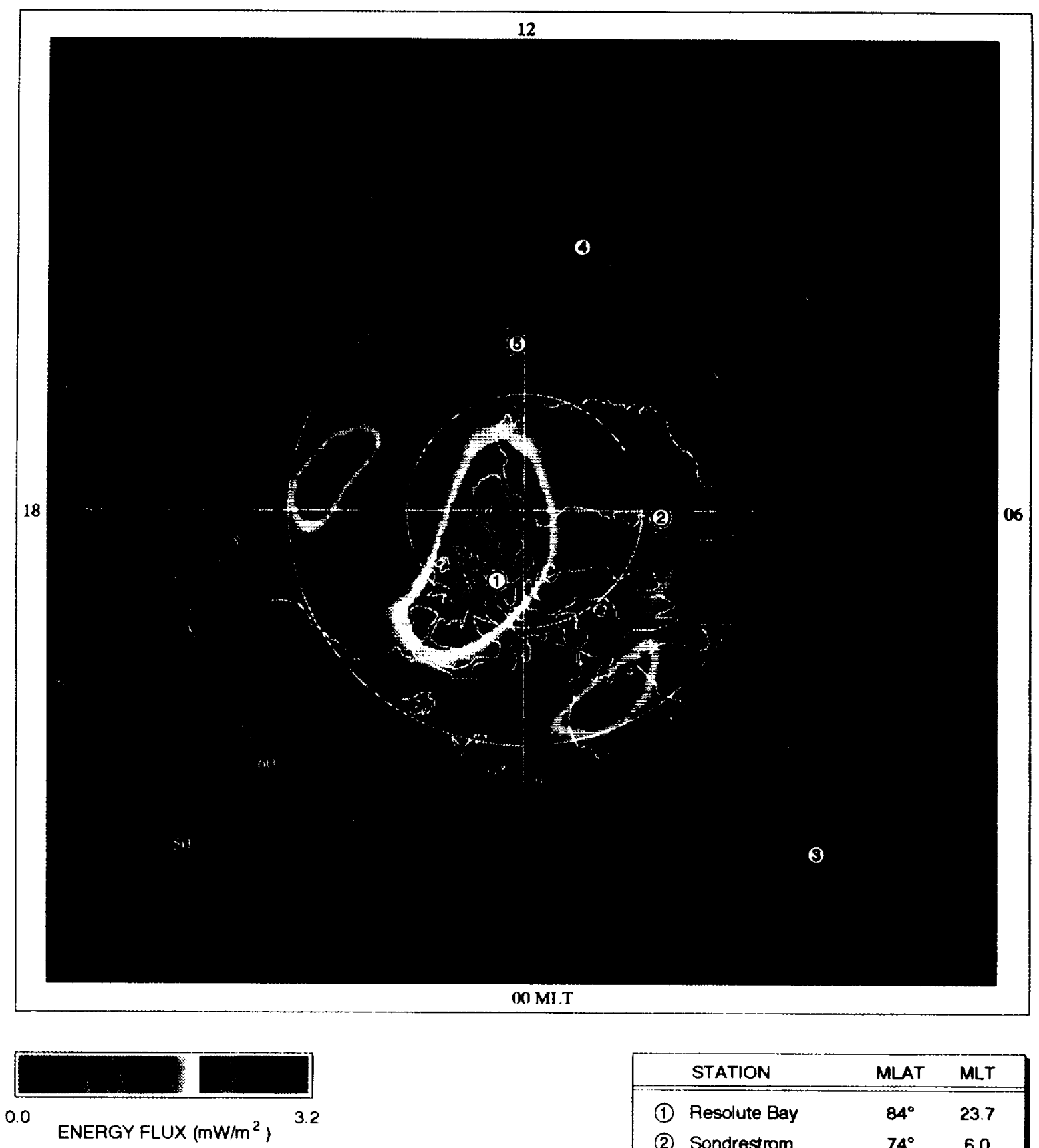

\begin{tabular}{|lll|}
\hline STATION & MLAT & MLT \\
\hline (1) Resolute Bay & $64^{\circ}$ & 23.7 \\
(2) Sondrestrom & $74^{\circ}$ & 6.0 \\
(3) Millstone Hill & $56^{\circ}$ & 3.5 \\
(4) EISCAT (TROMSO) & $67^{\circ}$ & 11.2 \\
(5) Svalbard & $75^{\circ}$ & 12.0 \\
\hline
\end{tabular}

FIGURE 19 The input of energy to the upper atmosphere in northern polar regions in winter under quiet geomagnetic conditions. The coordinates are magnetic latitude and longitude. Ground-based radar facilities for studying the polar ionosphere are identified. (Courtesy of James Vickrey, SRI.) 
temporal and spatial resolution. Field-widened versions of Fabry-Perot and Michelson interferometers allow large, nightside regions to be imaged over short time scales. For dayside measurements, highly improved spaceborne limb-scanning systems are available. Improvements in detector technology also give us the ability to image emissions in the near-infrared, providing the capability of mapping ion convection and neutral wind systems from both the ground and space.

New techniques are needed to determine global patterns of ionospheric currents, field-aligned currents, Joule heating, and neutral composition. One method of achieving this goal would be the use of a swarm of microsatellites in polar orbits spaced in local time to obtain nearly instantaneous global patterns of key parameters. Another approach would be to expand the network of ground-based incoherent scatter and other radars. The radars at Millstone Hill, Sondre Stromfjord, Goose Bay, and EISCAT have made great contributions to mapping the electric fields impressed into the auroral E region from the magnetosphere (Figure 19). The Europeans are building a radar on Svalbard (near the magnetic latitude of Sondre Stromfjord hut at a different local time). Construction of additional radars at strategic sites in the polar cap and around the auroral oval would yield a network capable of resolving the flow pattern of magnetic flux from the dayside of the Earth, across the pole, to the nightside, and of determining how this flux returns to the dayside at lower latitudes.

Available observational data in the lower-altitude regions of the ionosphere, where electrodynamic coupling is most effective, are exceedingly sparse. The reason for this lack of information is the inaccessibility of this altitude regime to conventional orbiting spacecraft. Current information from rockets and radars indicates that this region is highly structured. Again, the coupling processes between the magnetosphere, ionosphere, and atmosphere are strongly influenced by this region and its structure. Current ideas for a comprehensive survey of this region include spacecraft with highly eccentric orbits and a low-altitude perigee and instruments tethered to higher-altitude spacecraft. Whatever observational method is adopted, high-resolution measurements of electrodynamic parameters at these altitudes coordinated with higher-altitude, magnetically conjugate observations are essential to further understanding of this topic.

Important gaps in our knowledge of the dynamics of the upper atmospheres of Venus and Mars could be filled by observations of upper atmospheric winds using instruments such as a Fabry-Perot or a Michelson interferometer on spacecraft orbiting the planet. The chemistry and physics of the upper martian atmosphere could be addressed by orbiting instruments such as neutral and ion mass spectrometers and Langmuir probes. Theories about the ionospheres of the outer planets could be tested if ion composition measurements were made from any missions to those planets. 


\section{Plasma Processes That Accelerate Very Energetic Particles and Control Their Propagation}

\section{SCIENTIFIC BACKGROUND}

Energetic particles appear to be accelerated wherever plasma, magnetic fields, and motion are observed. Figure 20 summarizes the many sources of energetic particles in the heliosphere. The energies reached seem to depend on the scale of the object that accelerates them, although nowhere is the process fully understood. Because energetic electrons emit observable radio frequency synchrotron radiation, researchers know that energetic electrons and magnetic fields are common features of the Sun's corona, the planets, and galaxies everywhere. Solar energetic particles and galactic cosmic rays have much in common. They include electrons and nuclei over the entire periodic table accelerated to a wide range of energies-from less than $1 \mathrm{MeV}$ to over $10^{20} \mathrm{eV}$. The study of these particles provides a vital bridge between space plasma physics using in situ measurements and remote sensing of astrophysical objects where cosmic rays may have important dynamical consequences. Studies of point sources of gamma rays (e.g., active galactic nuclei and pulsars) have already led and will continue to lead to increased understanding of particle acceleration in other space environments. Science demands that we understand the acceleration of cosmic rays, the most extraordinary nonthermal process in the universe.

Solar energetic particles have been observed since before the dawn of the space era. As our ability grew to launch satellites outside of the Earth's local space environment, our knowledge of these solar-related events increased enormously. These solar particles are now known to span the energy range from solar wind plasma to greater than $10 \mathrm{GeV}$, have variable abundance and charge states, and appear to be accelerated by more than one process. There are short-lived, low-intensity events with widely varying compositional signatures associated with solar flares. There are also long-lived. very high intensity events with moderately similar compositional signatures associated with coronal shocks. In addition, energetic particles are further accelerated and their spatial distribution modified by shocks propagating through the heliosphere.

From balloon and satellite observations over the last 25 years. researchers know that cosmic rays fill our galaxy with an energy density comparable to that in the turbulent motion of the interstellar gas and in magnetic fields. Gamma-ray measurements by the Compton Gamma Ray Observatory (CGRO) and earlier satellites and ground-based radio observations have mapped their distribution throughout the galaxy. The high degree of isotropy of the cosmic rays as measured by ground-based air shower arrays at energies above $10^{12} \mathrm{eV}$ is an important constraint. Cosmic rays are hypothesized to originate in a large number of sources, probably supernova remnants. in a thin disk near the plane of the galaxy. Once accelerated they hecome highly mixed as they diffuse through the galactic magnetic fields and into the galactic halo.

Theories of stellar evolution and the synthesis of the elements reveal that most of the nuclei heavier than helium are formed in the interiors of stars. The energy released when lighter elements fuse into heavier ones supports stars against gravitational collapse. When this source of energy is exhausted, stars explode, hurling heavy 


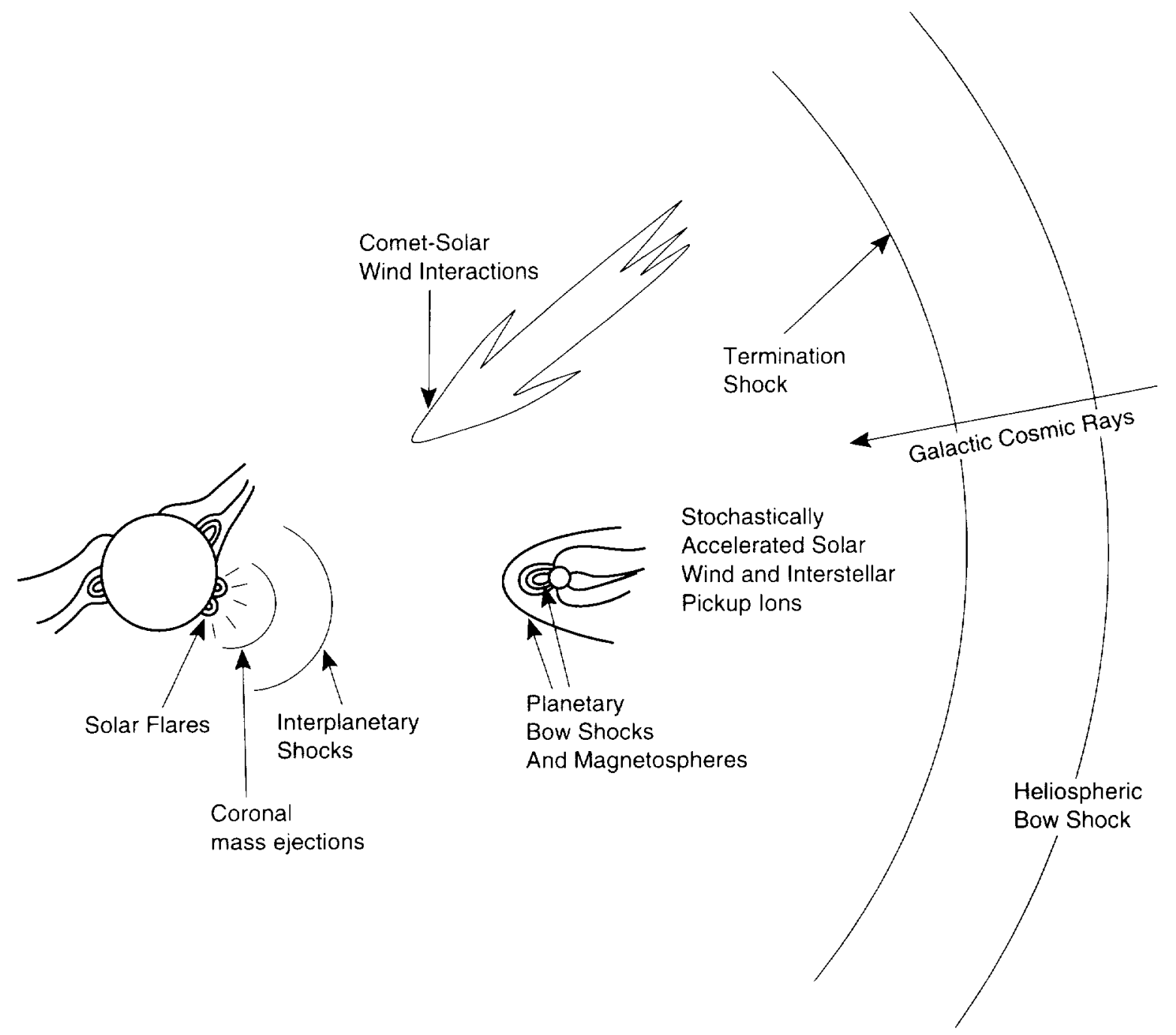

FIGURE 20 Sources of energetic particles in the heliosphere. 1Courtesy of Marcia Neugehauer, Jet Propulsion Laboratory.)

elements into the interstellar medium. The explosions create additional, even heavier elements that can be subsequently accelerated, perhaps by shocks from those same explosions, to cosmic-ray energies. Supernova explosions are the only sources capable of supplying the energy required to balance the leakage of cosmic rays from the galaxy as determined by estimating the lifetime of cosmic rays from the surviving fraction of radioactive ${ }^{10} \mathrm{Be}$ and other radioactive isotopes.

The energies of the galactic cosmic rays cover a range of more than 14 orders of magnitude, but how they are accelerated is unknown. Solar energetic particles are accelerated by solar flares and coronal shocks, processes believed to involve more than one mechanism. At the higher energies the processes of acceleration and propagation must involve interactions with the large-scale magnetic structures of the system. Measurements of the energy spectrum of each individual component of the solar energetic particles have been made by a variety of spacecraft throughout the solar system (IMPs, OGOs, Helios, ICE/ISEE-3, Pioneers, Voyagers, and so on). The spectra of galactic cosmic rays have been measured on space missions such as HEAO-3 and Spacelab-2 up to about $10^{12} \mathrm{eV}$; exploratory measurements by the JACEE group and on Russian satellites have been made up to approximately $10^{1+} \mathrm{eV}$. For the higher-energy cosmic rays, the principal information is the composite "all particle" spectrum, which comes from ground-based air shower detectors that measure the total energy arriving at ground level. 
Inference of composition from these measurements is currently only statistical; identification on an event-by-event basis is not possible.

New knowledge of the highly inhomogeneous nature of the galaxy (e.g., molecular clouds, supernova bubbles, and the highly variable plasma environment) from satellites like EUVE, Rosat, and CGRO has given rise to a new generation of models for the origin and acceleration of galactic cosmic rays. The models are based on the idea that particles are confined by magnetic fields to remain in the vicinity of shock waves produced by converging or overtaking plasma flows. These theories were developed to explain in situ observations of energetic particles near planetary bow shocks and shocks in interplanetary space; it has been demonstrated that heavy ions can gain significant amounts of energy in such an environment. Shock acceleration is probably important in the solar corona, but a different mechanism is probably responsible for accelerating solar flare particles in a magnetically closed environment. In some space environments, magnetic reconnection may be important in removing energy from the plasma and giving it to individual particles. On a galactic scale, hydromagnetic motion and reconnection must have a role in determining the evolution and shape of the magnetic fields, and the release of energy by reconnection is a candidate acceleration mechanism.

The spatial scales of interplanetary shocks are quite different from those in interstellar space. The question is whether or not the scale of interstellar shocks is sufficient to produce the observed cosmic rays. The detailed answers are believed to be accessible by extending the detailed composition measurements to cover the whole energy regime over which the mechanism is expected to operate. The critical features are expected to be significant deviations from power law spectra and predictable upper-energy cutoffs that are different for different species.

There are limits to these theories, however. They inadequately explain the acceleration of electrons, even though electrons are clearly accelerated in a variety of space plasma environments from planetary magnetospheres, to solar and interplanetary environments, to supernova remnants, pulsar winds, and active galactic nuclei. In addition, these theories are probably not sufficient to explain the origin of the most energetic particles-those above $10^{15} \mathrm{eV}$ where there is a kink in the observed energy spectrum, suggesting a significant change in the physics of the cosmic rays.

The sources of cosmic rays in the galactic disk have an important role in providing the energy that affects the dynamics of the galactic plasma. For example, the energy density of galactic cosmic rays is sufficiently high that they must have an important dynamical role in the generation of the galactic magnetic field. They inflate this magnetic field and the plasma linked to it to form a galactic halo and possibly drive a galactic wind (Figure 21), analogous to the manner in which the Sun provides the energy to drive the solar wind. Parallels can be drawn between the two systems, the heliospheric system consisting of the Sun located in interstellar space and the Milky Way galaxy located in intergalactic space. In both cases, there are important sources that input energy, extended magnetic field structures, traveling shocks that accelerate particles, and plasma pressure which drives winds. Are there also, in both cases, escape of particles from the system and entry of particles from outside the system? The loss of particles from the "galactic magnetosphere" is energy dependent, affecting the interpretation of source spectra and hence acceleration mechanisms. New theoretical work shows that the consequences of the storage and diffusion of energetic particles in the galactic halo have an observable effect on the lifetime of cosmic rays in the galaxy. The abundances of radioactive isotopes such as ${ }^{11} \mathrm{Be}$ and ${ }^{26} \mathrm{Al}$, at energies where the relativistic time dilation effects are important ( $>1 \mathrm{GeV} /$ nucleon), increase in a manner that depends on the speed of a galactic wind, thus allowing one to distinguish between convective and diffusive losses of particles from the galaxy.

Space physicists have little knowledge of the low-energy end of the cosmic-ray spectrum (below $100 \mathrm{MeV} /$ nucleon) in the galaxy because those particles are strongly affected by their interactions with the interplanetary magnetic field and the boundary structures of the heliosphere. Better understanding of those interactions is required to infer the interstellar spectrum, especially at energies from 1 to $300 \mathrm{MeV} /$ nucleon, and to apply that knowledge to models of cosmic ray transport elsewhere in the galaxy. In situ studies beyond the heliopause would measure directly the unmodulated spectrum and energy density of cosmic rays in interstellar space, and thereby establish their contributions to the ionization and heating of the interstellar medium, to the production of rare isotopes of cosmological interest, and to processes that include star production. Such studies could be carried out by an interstellar probe designed to pass through the termination shock and heliopause to explore the interstellar medium directly.

Cosmic rays enter the solar system carrying information about their origin and propagation through the galactic magnetic fields and interstellar matter. They are an accessible sample of matter from outside the imme- 


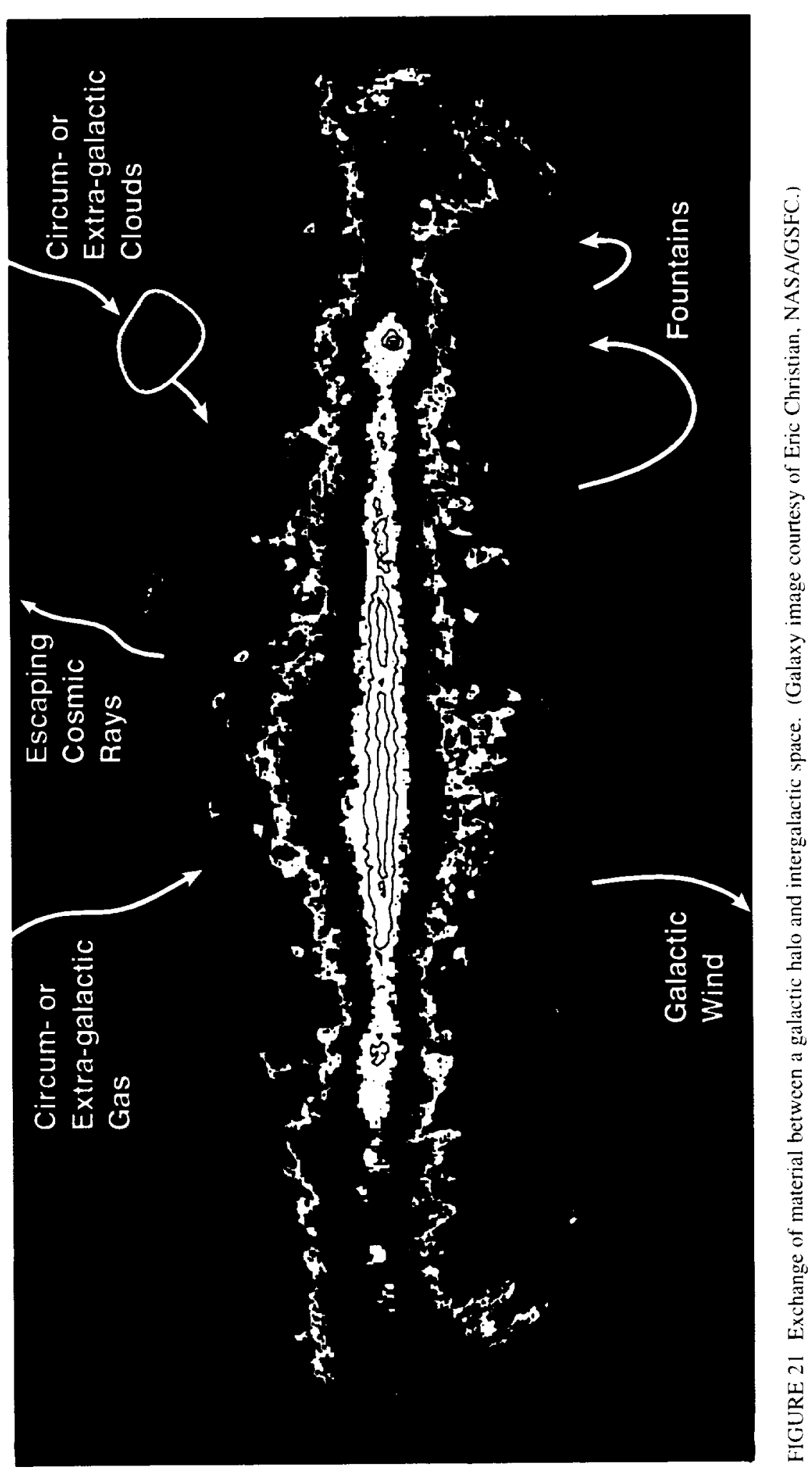


diate solar neighborhood. Similarly, solar energetic particles provide a sample of solar material that contrasts with that obtained from the solar wind. In all cases, systematic and intriguing patterns emerge in the differences in elemental and isotopic abundance patterns between these samples and those observed in planetary and meteoritic material. Much has already been learned about these patterns from HEAO-3 experiments and from isotope measurements, first on ISEE- 3 and now on SAMPEX and Ulysses. The high statistical accuracy of the measurements of solar flare particles, the anomalous component, and galactic cosmic rays on ACE will round out these studies for nuclei with atomic numbers less than 30 and kinetic energies less than $400 \mathrm{MeV} /$ nucleon. Understanding abundance differences in these samples of matter is vital to the study of solar system evolution and to answering questions relating to the uniqueness of the terrestrial environment.

Following ACE, it is important to extend high-resolution composition studies to the upper two-thirds of the periodic table. Cosmic rays with atomic numbers greater than 30 (often called the ultraheavy nuclei) are made predominately by the " $r$ " (rapid) and " $s$ " (slow) neutron capture processes that occur in markedly different stellar environments. The r-process occurs in explosive environments where the neutrons are added more rapidly than the time scale for decay, and the s-process occurs where neutrons are added more slowly. Measurements of individual clements and isotopes of these nuclei are required to establish the relative contributions of these processes to the nucleosynthesis of cosmic ray material and to study the evolution of the galaxy. Studies with HEAO-3 and Ariel 6 found an overabundance of r-process nuclei made primarily in supernova explosions. An accurate measurement of the uranium-to-thorium ratio would give a mean age of the heaviest cosmic rays from the time of nucleosynthesis in much the same way that those nuclei are used to measure the age of rocks on Earth. The presence of other transuranic elements with shorter half-lives would be the signature of a recently synthesized r-process component in the cosmic rays.

The composition of the source of cosmic rays cannot be understood without understanding preferential acceleration, fractionation, and propagation processes. For example, one process that appears to be important in explaining the differences between the elemental composition of cosmic rays and solar-system material, and also between the solar wind and the solar photosphere (to the extent that such differences have been measured), depends on the first ionization potential (FIP) of the elements. Easily ionized material has an enhanced abundance in both the cosmic radiation and some types of solar wind flows. But the FIP effect is probably not the whole story. As they traverse the matter in the acceleration region and in the interstellar medium, the cosmic-ray nuclei may undergo nuclear interactions that fragment them and change the composition of the sample. These effects can be calculated if the necessary nuclear parameters are known and if a suitable model is adopted for the propagation of the particles. Strong experimental and theoretical efforts are needed to resolve the major uncertainties that still exist in making such calculations.

Important information about cosmic ray acceleration is obtained from the study of the energy spectra of secondary cosmic rays. which are energetic particles produced by collisions with interstellar material. Both the spatial distribution and energy spectra at "injection" of secondary cosmic rays can be calculated with far fewer assumptions than are required for the cosmic rays in general. Much work has been done with secondary nuclei, such as lithium, beryllium and boron, but the results to date have not been conclusive, and more data on heavier secondary particles are clearly needed. Positrons and antiprotons are the least studied of the light secondary particles, and are unique in that they cannot coexist with matter of any significant density. Therefore they cannot be produced by some two-stage processes proposed for the generation of heavier secondaries. Initial observations of antiprotons suggest an abundance larger than that expected from the studies of heavier nuclei. If confirmed, this result will indeed challenge the simple expectation (Occam's razor) that all cosmic rays are accelerated by the same mechanism. In any event, the antiproton flux must be measured over an extended energy range because it will yield information on cosmic ray propagation that cannot be obtained from heavier nuclei alone.

The only fact clearly established about cosmic ray positrons is that, over a significant energy range, they are less abundant than electrons. This, however, is a fundamental observation, since an origin solely from interstellar interactions would produce a composition dominated by positrons. The implication is that electrons are indeed accelerated to cosmic ray energies. Explaining energetic electrons remains a major problem for theories of cosmic ray acceleration, even as radio observations of distant galaxies confirm that they are ubiquitous. Detailed determination of the spectra of electrons and positrons of all energies is greatly needed.

Positrons can also contribute to cosmic rays as primary particles. Decay of radioactive supernova ejecta releases a large number of positrons at energies well above the injection threshold of most cosmic ray acceleration models. The composition and distribution of this radioactive material can be determined through observation of 
the gamma rays that it emits. Either detection or nondetection of these positrons as energetic cosmic rays will make a fundamental statement regarding the cosmic accelerator.

Pulsar winds could also produce energetic positrons. It is thought that the plasma in such winds is produced by an electromagnetic cascade in the ultrastrong fields in the pulsar magnetosphere and therefore consists of positive and negative electrons in equal numbers. The energy in the wind flow itself could be at the TeV level, with even higher energies possible in subsequent acceleration at a termination shock.

Apart from interaction products, antibaryons have never been observed in nature. No fully satisfactory theory has been advanced to support the extension of this relatively local (i.e., local cluster of galaxies) observation to the universe as a whole. Direct experimental tests for the existence of large domains of antimatter in the universe are possible by searching for antimatter in the small fraction of the cosmic radiation that could be of extragalactic origin. The most sensitive current searches from balloon experiments place upper limits in the range of a few antiparticles in $10^{4}$ or $10^{5}$ helium nuclei, whereas if there are equal numbers of matter and antimatter galaxies within 30 to $300 \mathrm{Mpc}$ of Earth, we might expect to find antinuclei in the cosmic rays at a level of a few parts in $10^{5}$ or $10^{6}$. This number is highly uncertain; it depends on whether or not there is strong galactic modulation that excludes low-energy particles in a manner analogous to the way the solar wind excludes low-energy galactic particles.

Questions about the origin of cosmic rays have become more focused and detailed over the past decade, even though there is still much to learn. Phenomenological models have begun to be replaced with more physical models. Space-based measurements have shown the power of making statistically significant measurements above the atmosphere. The nuclear interaction cross sections necessary for interpreting the data are being better measured. Spacecraft approaching the outer boundary and the polar regions of the heliosphere are acquiring new data with which to address the question of how cosmic rays enter the heliosphere.

During the coming decade, studies of the galactic cosmic rays and their interactions with the underlying matter and plasma should lead to detailed understanding of their acceleration and propagation. The principal current questions in cosmic ray research are the following:

- How are solar energetic particles and galactic cosmic rays accelerated? Do supernova or stellar-wind shocks provide the bulk of the cosmic ray energy? Is there more than one mechanism operating? How are particles injected into the accelerator?

- What are the abundances of the sources of the cosmic ray elements and isotopes, and how do they differ from solar system abundances? What are the nuclear processes that produced this material? Do the abundances show evidence of galactic evolution since the formation of the solar system? What are the preferential selection and fractionation processes that modify the abundances?

- What time has elapsed between synthesis of the elements and their acceleration? How long has it been since the heaviest elements were synthesized?

- How do cosmic rays interact with matter and magnetic fields on galactic temporal and spatial scales?

- How are energetic particles confined in both ordered and chaotic magnetic fields? What are the mechanisms by which they leak out, and what determines the rate at which this escape occurs?

- Is there a galactic wind? If so, is it driven by cosmic ray pressure? Does it convect particles out of the galactic magnetosphere?

- Is there an extragalactic component of the cosmic radiation? If so, can researchers find evidence for primordial antimatter in this sample?

\section{CURRENT PROGRAM}

The elemental composition and the isotope ratios for all elements through nickel will be measured by a sequence of instruments on SAMPEX. Ulysses, Wind, and ACE at energies up to a few hundred MeV/nucleon. Those measurements should lead to a much better understanding of the systematics of fractionation processes and will provide new information on galactic evolution since the formation of the solar system.

Balloon-borne experiments are making progress in a number of specific areas. Measurements are currently under way to study positrons and antiprotons over a limited energy range. Balloon-horne experiments for exploratory measurements of isotopic composition above $0.5 \mathrm{GeV} /$ nucleon for elements up to oxygen, for studying transiron isotopes, and for studying ultraheavy elements are currently planned. Nuclei beyond iron in the periodic table 
have been studied previously on Ariel 6, HEAO-3, and LDEF. Measurements of the energy spectra of the few most abundant elements have been carried out to energies beyond $10^{14} \mathrm{eV}$ on balloons, HEAO-3, and Spacelab-2. Searches for antihelium and heavier antinuclei are being carried out; the expected sensitivity would lead to detection of one antihelium nucleus per $10^{5}$ to $10^{6}$ helium nuclei.

Extensive air shower experiments show that a major feature in the cosmic ray spectrum exists at an energy between $10^{15} \mathrm{eV}$ and $10^{1 \mathrm{~h}} \mathrm{eV}$. So far, inconclusive but promising attempts are being made to derive the elemental composition in that energy range from air shower data.

A program is currently under way to measure the nuclear parameters that describe the propagation of cosmic ray nuclei through the matter in the interstellar medium. Without the nuclear parameters, composition measurements are difficult to interpret or compare with model predictions. Recent work indicates that these parameters are energy dependent over a greater range than previously thought. Those cross sections are absolutely essential in order to deduce the true nature of the cosmic radiations as accelerated. They should be made with a precision that will allow the measurements being made on the satellites mentioned above to be interpreted without introducing significant additional error.

\section{FUTURE DIRECTIONS}

An important new generation of isotope spectrometers recently launched (Ulysses, SAMPEX, and Wind) or soon to be launched (ACE) will join those on Voyager in making extensive measurements of the elemental and isotopic compositions and charge states of cosmic rays throughout the heliosphere. This important new set of observations will satisfy long-standing recommendations of earlier National Research Council committees. The recently developed capability to fly high-altitude balloons for 10 days or more makes it possible to begin carrying out some of the important measurements that researchers can currently identify. Other objectives require measurements in space where they can be made without contamination by atmospheric background and where significant statistics can be gathered to make the precise measurements necessary to distinguish between models.

The structure in the total cosmic ray energy spectrum at energies above $10^{14} \mathrm{eV}$ is probably related to variations in composition. Furthermore, the energies at which that structure occurs are quite close to the energies expected on the basis of the lifetimes of supernova shocks. It is now technically feasible to extend cosmic ray composition measurements made from balloons up to energies approaching $10^{15} \mathrm{eV}$ and similar measurements made from ground-based (air shower) observatories down to energies of $10^{14} \mathrm{eV}$. By an aggressive but completely practical program of research, a full order of magnitude of overlap between those two techniques can be obtained in the coming decade. Observations using a large calorimeter on a space-based platform would test theories concerning the acceleration of galactic cosmic rays in supernovae shocks and provide details on the transformations of the source spectrum and composition that take place between the acceleration and the observation of the particles.

A large magnetic spectrometer in space will be required to make isotope measurements at energies above $0.5 \mathrm{GeV} /$ nucleon. In particular, measurements of the radioactive nuclides, ${ }^{116} \mathrm{Be},{ }^{26} \mathrm{Al}$, and perhaps ${ }^{36} \mathrm{Cl}$, at energies above $1 \mathrm{GeV} /$ nucleon will provide a test for the existence of a galactic wind and bound its velocity. Because of the relativistic time dilation, the energy dependence of the surviving fraction of those radioactive nuclei depends on whether the loss mechanism from the galaxy is purely diffusive or also includes convective terms. While the first steps toward this objective can be met by balloon-borne observations, completing the scientific objective ultimately requires measurements of high statistical precision made outside the atmosphere.

It is important to complete the characterization of cosmic rays heavier than nickel, since abundances of only about a third of those nuclei have been measured. Of particular importance are the trans-iron elemental abundances of odd- $Z$ nuclei and the very rare radioactive actinide elements that can be used to determine the age of this sample of matter. Long-duration balloon flights of detectors currently being developed should enable the measurement of abundances of the lower-charge trans-iron nuclei up through strontium $(Z=38)$. Instruments flown on satellites are required to make definitive measurements of the heaviest element abundances, in particular the actinides.

The spectra of positrons ( $10 \mathrm{MeV}$ to $100 \mathrm{GeV}$ ) and of antiprotons ( $1(10 \mathrm{MeV}$ to $100 \mathrm{GeV}$ ) must be measured to determine where those particles are created and how they are accelerated. Between approximately 3 and $30 \mathrm{GeV}$, the necessary cosmic ray positron and antiproton measurements can be obtained by continued support of 
currently planned and new long-duration balloon instrumentation. For lower and higher energies, atmospheric secondary particles prevent definitive measurements from balloons, particularly for positrons at energies below $2 \mathrm{GeV}$. Technology has now evolved to the point that the required mass of a positron or antiproton detector is easily compatible with the payload capacity of small launch vehicles such as the Pegasus and the Taurus.

Flights of instruments on long-duration balloons for 10 days should be capable of extending the search for antihelium nuclei to 1 part in $10^{7}$. Measurements in space would extend the sensitivity by yet another factor of 30 or more. A positive detection, however unlikely, would have profound implications for fundamental physics and cosmology. 



\section{Part III}

\section{Research Strategy}

Part III outlines the four common thrusts in the committees' recommended strategy for future U.S. research in space physics, and it summarizes the more specific research efforts recommended to address the five key scientific topics that the CSSP and CSTR believe should be and will be at the forefront of research in space physics during the next decade. Those five topics, discussed in detail in Chapters 1 through 5, are listed in Box 3. Under each

topic, the research activities recommended by the committees are entered in the order of their priority (i.e.. the highest priorities are listed first). 


\title{
Box 3 \\ Prioritized Listing of Research Activities in Five Topics Identified as Key Areas for Study in the Next Decade
}

\author{
Mechanisms of Solar Variability
}

- Use helioseismology to study the structure and dynamics of the solar surface and interior over a full solar cycle.

- Assure continuity of total and spectral irradiance measurements, supported by spatially resolved spectrophotometry, to investigate correlations between solar magnetic activity and solar output variations.

- Measure high-energy radiation and particles from flares and coronal mass ejections with good angular resolution, good spectral resolution, and wide spectral coverage to determine what drives each of these phenomena and how they contribute to the solar output at high energies.

- Observe surface magnetic fields, velocities, and thermodynamic properties with enough spatial resolution $(<150 \mathrm{~km}$, with an ultimate goal of $<100 \mathrm{~km})$ to study small-scale structures such as flux tubes.

- Make global-perspective measurements of the solar surface magnetic and velocity fields and solar oscillations to measure the three-dimensional structure and long-term evolution of active regions and to detect weak but coherent global oscillations.

- Measure active regions with angular resolution of $\sim 1$ arc sec and temporal resolution of $\sim 10$ min for a duration of $\sim 10$ days without nighttime gaps to determine their magnetohydrodynamic history of emergence, development, and decay.

\section{The Physics of the Solar Wind and the Heliosphere}

- Continue to obtain and synthesize the data from the present constellation of heliospheric spacecraft (Voyager, Ulysses, Wind, and ACE) and from the interplanetary cruise phases of planetary missions in order to characterize the global and solar-cycle-dependent properties of the heliosphere and its interaction with the interstellar medium.

- Carry out in situ observations of the solar corona to explore and characterize the region of acceleration of the solar wind plasma and the physical processes responsible for that acceleration.

- Obtain new types of data required to reveal the mechanisms responsible for the transport of energy from the solar surface into the chromosphere and corona to understand how these are heated.

- Carry out stereo imaging of the solar corona to reveal the three-dimensional structure of coronal features without the ambiguity caused by integration along the line of sight.

- Develop and use techniques for the remote sensing of the coronal magnetic field in order to improve knowledge of the acceleration of the solar wind and of the initiation of coronal mass ejections.

- Make in situ measurements of the outer heliospheric boundaries and the interstellar medium with instruments specifically designed for those purposes.

\section{The Structure and Dynamics of Magnetospheres and Their Coupling to Adjacent Regions}

- Reap the full scientific potential of the International Solar-Terrestrial Physics program and its coordinated programs to advance understanding of the transport of mass, momentum, and energy throughout the solar wind, and the magnetosphere and ionosphere systems.

- Simultaneously image the plasma and energetic particle populations in the aurora, plasmasphere, ring current, and inner plasma sheet to study the global structure and large-scale interactions of magnetospheric and ionospheric regions during different levels of solar and geomagnetic activity.

- Maintain the full complement of particle and field instruments on current and future planetary missions to gain increased understanding of the formation and dynamics of diverse magnetospheres and ionospheres.

- Further develop and exploit ground-based facilities that image the ionospheric manifestations of solar wind/magnetosphere coupling processes to complement the magnetospheric imaging initiative aimed at studying the global properties of the magnetosphere.

- Explore Mercury's magnetosphere to understand the role of an ionosphere in coupling between the solar wind and planetary magnetospheres.

- Target localized regions that require greater understanding of the small-scale physical processes occur- 
ring there with high-resolution, multispacecraft measurements that take advantage of new smaller, lighter, more capable instruments and more sophisticated data-compression schemes.

\section{The Middle and Upper Atmospheres and Their Coupling to Regions Above and Below}

- Exploit the exciting new capabilities of UARS, FAST, and CEDAR to provide the foundation for future advances in our understanding of the middle and upper atmospheres.

- Investigate the reaction of the middle and upper atmospheres to upward propagating waves and energy inputs from space so that the sources of important features such as the quasi-biennial and semiannual oscillations and the causes of mesosphere/lower-thermosphere structure and variability can be understood.

- Study the long-term variations in the middle and upper atmospheres using a combination of consistent long-term satellite and ground-based measurements together with three-dimensional radiative-chemicaldynamical modeling to understand natural and anthropogenic changes in these regions.

- Develop methods to observe the time-dependent electrodynamics operating on microscales to global scales, both in the upper atmosphere-ionosphere-magnetosphere coupling regions so that feedback processes can be characterized, and in the regions above thunderstorms so that the effects of electrified clouds on the "global circuit" and on middle atmosphere chemistry and energetics can be characterized.

- Take advantage of opportunities to include carefully chosen, appropriate instruments on planetary orbiter missions to make measurements critical to understanding planetary aeronomy and its relation to terrestrial aeronomic processes.

\section{Plasma Processes That Accelerate Very Energetic Particles and Control Their Propagation}

- Complete the observations from the current and planned network of interplanetary spacecraft to study particle acceleration, fractionation, and transport.

- Extend direct composition measurements to $10^{15} \mathrm{eV}$ to probe the limits of acceleration and trapping mechanisms.

- Measure abundances of radioactive isotopes above $1 \mathrm{GeV} /$ nucleon to search for evidence of an extended galactic magnetosphere and wind.

- Measure the spectra of positrons (10 MeV to $100 \mathrm{GeV}$ ) and antiprotons (100 MeV to $100 \mathrm{GeV}$ ) to determine where those particles are created and how they are accelerated.

- Measure isotope abundances for nuclei heavier than nickel and elemental abundances through the actinides to probe the plasma regions where the nuclei are synthesized and to measure the time scales involved.

\section{RECOMMENDED RESEARCH ACTIVITIES}

In generating the prioritized lists of recommended research activities, the CSSP and CSTR considered the following issues:

- Importance. What is the importance of a particular research activity to answering the questions posed for the related major topic? Is the research effort highly likely to resolve one or more important issues? Does it address a major piece of the puzzle?

- Timeliness. Is the technology necessary to conduct the activity in hand or on the near horizon, and is the activity ripe for progress if initiated in the coming decade? Is the activity time-critical or a unique opportunity? Does the activity take advantage of the availability of new tools?

- Breadth. Would the results of the research activity provide fundamental understanding with broad applicability? Would the results be applicable to other aspects of space physics, to space science in general, or to other branches of science?

- Practical relevance. Is the research activity highly relevant to the issue of the effects of solar variability on the Earth? 
- Balance. Would implementation of the activity help redress the current imbalancel between "big" and "little" science and between planning, development, and pure science activities in space physics?

It is important to use with care and common sense the priorities recommended in this report. In many cases the activities are not really in direct competition; they address different scientific issues, their funding agencies may be different, and the amount of money needed may be very different. A priority rank is not an absolute attribute; it is a function of the question being asked. If one carries out only the highest-priority activity that addresses a particular question, the question will not be answered unambiguously or completely. Lower-priority activities can often be justified over higher-priority ones if they are more affordable in the current environment or if they better meet some other goal such as balance between subdisciplines, research techniques, institutions, or other parameters that were not taken into account in the present study. Often two or more lower-priority items taken together can be as important as a single higher-priority item. Also to be factored in are new or unforeseen developments or results in science and technology. But, other things being equal, the committees believe that the scientific precedence for space physics research is that given in Box 3.

\section{RECOMMENDED RESEARCH EMPHASES}

The recommended research activities listed in Box 3 are elements of the overarching goal of achieving greater understanding of the physical processes that underlie the observable phenomena of space physics. These rather detailed lists of diverse research efforts incorporate four common elements that the CSSP and CSTR consider to be the most important emphases for space physics research in the next decade:

1. Complete currently approved programs. Successful completion of the current programs is the committees' highest-priority objective, given that the committees endorse the previous scientific strategies for which those programs were proposed and approved. Successful completion of the current efforts will provide the foundation to which new elements will be added to make scientific progress. In making successful completion of current undertakings the first priority, the committees emphasize that space physics programs do not end with the launch of a spacecraft or the construction of a new facility. For an effort to be worthwhile, the data must be acquired, carefully analyzed, and interpreted. Each of those steps, followed by theoretical assimilation of the results, is necessary to gain the insights needed to advance the science. Furthermore, giving increased emphasis to data collection and interpretation will also help improve the balance between large- and small-scale efforts in space physics. It must also be recognized, however, that the high priority given to successful completion of established programs does not imply blanket endorsement of extended missions. Each mission extension must compete on its own merits with other ways in which the same resources could be spent. The committees" specific recommended actions for experiment operation and data analysis and interpretation are as follows:

a. Completing the diverse components of the International Solar-Terrestrial Physics program. This includes the full-term support of mission operations and data analysis, with effective coordination of the observational and data-analysis campaigns involving the many spacecraft, both U.S. and foreign, and supporting ground-based observatories. The flight projects should cooperate with the several organizations or programs (GEM, STEP, and the IACG) that have been put in place for that purpose.

b. Enhancing data analysis and interpretation efforts for other space missions that have been launched recently or are currently in development. These include Yohkoh, SAMPEX, FAST, ACE, SOHO, Galileo, and Cassini. Adequate support is also required for the successful fulfillment of the objectives of NSF's CEDAR, GEM, and SUNRISE programs.

c. Streamlining mission operations to help keep extended missions going as well as minimize operations costs for new missions.

d. Carrying out extended missions for the uniquely placed Voyager (to the greatest possible heliocentric distance) and Ulysses (through the solar polar passes at solar maximum).

e. Continuing support of those ground- and space-based observations that require improved statistics or the acquisition of data over a range of solar-cycle conditions. At issue are (1) gap-free, space-based observations of the variability of solar luminosity, (2) solar neutrino and helioseismological observations, and (3) monitoring of the composition, dynamics, and temperature of the middle and upper atmospheres to obtain a baseline for future 
studies of climate changes, together with (4) monitoring and prediction of space "weather" in support of a wide range of research and operational activities.

f. Enhancing the effectiveness of some of the longer-duration programs by soliciting new ideas and analysis techniques from guest investigators and by ensuring easy access to archived data resulting from the various programs for use in "small science" research tasks.

2. Exploit existing technologies and opportunities to obtain new results in a cost-effective manner. The technology is already in place to take the next observational steps required to address many of the important questions in space physics. Both spacecraft and instrument technology are available to complement point-by-point in situ measurements of the magnetosphere with global imaging and to carry out a multi-spacecraft mission capable of establishing a baseline for studies of changes in the middle and upper atmospheres while separating spatial from temporal effects in those regions. Measurement of solar irradiance variations can also be carried out using current instrument designs. Other objectives can be addressed effectively by inclusion of instruments for space physics research on missions of opportunity, such as NASA's planetary missions and Department of Defense spacecraft, or through international collaborations. On suborbital platforms such as rockets and balloons or on the ground at new sites such as in the polar cap. operation of existing types of instruments can allow unique studies of some aspects of magnetosphere-ionosphere-atmosphere coupling. The advent of new networking, telescience, and other collaboration methods allows real-time display of data from widely separated sensors. Exploiting this technology allows experiments and ohservations to be optimized while reducing costs.

3. Develop the new technology required to advance the frontiers of space physics. We must continue to push the limits of technology in order to achieve other high-priority objectives or to lower the cost of projects that are already technically feasible. The technology required to approach the Sun ever more closely will open one of the most exciting frontiers of space science. New spacecraft using lightweight structures and miniaturized electronics, and balloon technology allowing 8- to 20-day flights around the South Pole, should enable some highpriority space physics measurements to be made inexpensively in the near term. A good start has been made in the development of the new technology required for the study of high-energy radiation and particles from transient solar events with high angular and spectral resolutions and wide spectral coverage. New techniques for fabrication of lightweight optics are becoming available from the former Soviet Union. Advanced instruments for the analysis of the rarest components of the cosmic radiation such as very heavy or very energetic particles must continue to be developed and flight-tested on long-duration balloons that can carry heavier payloads than are possible with current balloons. Small, capable instruments must be developed to enable affordable in situ measurements of the solar corona, the distant heliosphere, and some regions of planetary magnetospheres. Advances are also required in (a) infrared instrumentation and a large-aperture infrared-capable solar telescope, (b) second-generation instruments for magnetospheric imaging with greater sensitivity, energy and composition discrimination, and angular resolution, and (c) instrumentation for exploring the regions between those altitudes reached by balloons and by satellites.

As already mentioned, one of the conclusions of this report is that the highest near-term priority for space physics is the successful completion of the ISTP program and thorough correlation and analysis of the diverse sets of data that it will generate. That recommendation is entirely consistent with the current emphasis on the development of space technology that can be transferred to other sectors of the economy. The operations and data analysis phase of ISTP is a good opportunity for the development and use of new technology for experiment operations, automated or remote operations of ground-based scientific facilities, data distribution and archiving, and data visualization and other aids to interpretation.

4. Support strongly the theory and modeling activities vital to space physics. As emphasized in previous reports, ${ }^{2-5}$ theory and modeling complement dati analysis as vital components of all the subdisciplines of space physics research. In the coming decade, special attention or emphasis should be given to the following:

a. Recognizing that synergy between observations, modeling, and theory provides the optimum way of addressing the principal questions in space physics;

b. Making numerical simulations of space physics systems more realistic by extending them to three dimen- 
sions, longer time durations, and a greater range of scale sizes, and including additional interacting physical and chemical processes;

c. Ensuring that space physicists have ready access to state-of-the-art computational facilities, given that modeling relevant processes will require an order-of-magnitude increase in computer throughput;

d. Exploiting new insights gained from theory, especially the theory of nonlinear processes. Such work has already been started, with the development of new ways of looking at nonlinearities. Some problems, such as understanding the solar dynamo, require totally new theoretical insights, as opposed to extended modeling or simulations. It is possible that such insights might result from plasma-physics experiments performed in terrestrial laboratories; and

e. Revisiting earlier efforts to predict solar activity, such as coronal mass ejections and flares, using simulations combined with solar observations.

Although the four areas of emphasis summarized above are in priority order, it is absolutely essential that progress be made in all four. The space physics community must reap the benefits of what has already been built and paid for. Space physicists must take advantage of existing capabilities to make new types of measurements in a cost-effective way. Researchers must also develop and use the technology required for future progress. Finally, space physicists must continuously digest the new data, decide what those data mean, and keep fine-tuning and reviewing the scientific questions that drive the research.

\section{REFERENCES}

1. Board on Atmospheric Sciences and Climate and the Space Studies Board. National Research Council. A Space Physics Paradox: Why Has Increased Funding Been Accompanied by Decreased Effectiveness in the Conduct of Space Phwics Research?. National Academy Press, Washington, D.C., 1994.

2. Space Studies Board and the Board on Amospheric Sciences and Climate. National Research Council. Assessment of Programs in Solar and Space Phisics-1991, National Academy Press, Washington. D.C.. 1991.

3. Space Science Board. National Research Council, Solar-System Space Physics in the 1980's: A Research Strategy. National Academy of Sciences. Washington. D.C.. 1980.

4. Space Science Board, National Research Council, The Role of Theory in Space Sicience, National Academy Press, Washington, D.C.. 1983.

5. Space Science Board, National Research Council, An Implementation Plan for Priorities in Solar-System Space Physics. National Academy Press, Washington. D.C.. 1985. 


\section{Appendix}

\section{Glossary}

ACE

ACRIM

ADEOS

Akebono

AMPTE

Ariel 5

Ariel 6

AU

Cassini

CEDAR

CFCs

CGRO

Cluster

CMEs

CoMStOC

CRRES

DE

DMSP

EISCAT

Envisat

EOS

ERB

ESA

EUVE

Advanced Composition Explorer, scheduled for launch in the late-1990s Active Cavity Radiometer Irradiance Monitor Japan's Advanced Earth Observing Satellite, scheduled for launch in 1995 Japanese spacecraft, launched in 1989, for studying the inner magnetosphere Active Magnetosphere Particle Tracer Explorer-NASA/German/U.K. three-satellite mission to study the access of solar wind ions to the magnetosphere, as well as the convectivediffusive transport and energization of magnetospheric particles British x-ray astronomy satellite, launched in 1974 British satellite launched in 1979 to study the ultraheavy component of primary cosmic rays Astronomical unit - the mean distance between the Earth and the Sun

NASA-ESA mission to Saturn, scheduled for launch in 1997

Coupling of Energy and Dynamics between Atmospheric Regions program Chlorofluorocarbons

Compton Gamma Ray Observatory, launched from the shuttle in 1991

ESA-NASA multisatellite mission, scheduled for launch in 1996

Coronal mass ejections

Coronal Magnetic Structures Observing Campaign

Combined Release and Radiation Effects Satellite - a NASA-Department of Defense mission launched in the mid-1980s

FAST Fast Auroral Snapshot Explorer-NASA small explorer scheduled for launch in 1995 
FIP

Flare Genesis

Freja

Galileo

GALLEX

GEM

Geotail

GGS

GOES

GONG

HEAO-3

Helios

HILAT

HIREGS

Huygens

IACG

ICE (ISEE-3)

ILAS

IMP-8

Interball-Aurora

Interball-Tail

ISEE

ISTP

JACEE

LDEF

$\operatorname{Max} 91$

Meteor-3

MI

MSX

NDSC

NIMBUS

OGO

P78-1

Pioneer

Pioneer Venus
First ionization potential

Balloon-born solar telescope and magnetograph launched in Antarctica

Swedish-German satellite to study the interaction of the magnetosphere with the upper atmosphere and ionosphere-launched in 1992

NASA Jupiter orbiter, launched in 1989 and scheduled to go into orbit about Jupiter in 1995 Gallium Experiment-European solar neutrino detector located in Italy

Geospace Environmental Modeling program

Japanese-U.S. mission to study magnetospheric processes in distant geomagnetic tail Global Geospace Science program

Geostationary Operational Environmental Satellite--a series of NOAA meteorological satellites

Global Oscillation Network Group_ground-based helioseismology experiment employing multiple observing sites around the world

NASA's third High Energy Astrophysical Observatory, launched in 1979

A pair of German-U.S. spacecraft with perihelion of $\sim 0.3$ AU, launched in 1974 and 1976

A polar-orbiting satellite

High Resolution Gamma-ray Spectrometer-a 1992-1993 long-duration balloon flight experiment from Antarctica

Titan atmospheric probe-ESA's contribution to the Cassini mission

Interagency Consultative Group

International Cometary Explorer (formerly International Sun/Earth Explorer-3), launched in 1978

Instrument on the Japanese ADEOS

NASA's eighth Interplanetary Monitoring Platform, launched in 1972

Russian spacecraft for studying the auroral zones-launch expected in 1995

Russian spacecraft for studying the geomagnetic tail-launch expected in 1995

International Sun-Earth Explorer-a NASA-ESA series of three spacecraft launched in 1977 and 1978

International Solar-Terrestrial Physics program

Japanese-American (Coordinated) Emulsion Experiment-a balloon-borne cosmic ray experiment

Long Duration Exposure Facility, launched on the shuttle in mid-1980s

Coordinated program for studying the Sun during the solar cycle maximum in 1991

The Russian satellite carrying NASA's TOMS experiment, launched in 1991

Magnetosphere-ionosphere coupling

Midcourse Space Experiment-Department of Defense satellite scheduled for launch in 1995

Network for Detection of Stratospheric Change-the aim is to obtain a continuous, wellcalibrated data record for the stratosphere, for use in calibrating satellite measurements A series of U.S. atmospheric research satellites

Orbiting Geophysical Observatory

A Department of Defense solar observation spacecraft, launched in 1979

A series of NASA planetary and heliospheric spacecraft, launched in the 1960s and 1970s A NASA program to study Venus-two spacecraft, a bus with multiple atmospheric probes and an orbiter, both launched in 1978 
Polar A NASA contribution to ISTP, scheduled for launch in 1995

Polar BEAR Polar-orbiting satellite

QBO Quasi-biennial oscillation

RISE Radiative Inputs from the Sun to the Earth program

RoSAT Roentgen satellite-a German-U.S.-U.K. gamma/x-ray satellite launched in 1990

SAGE Soviet-American Gallium Experiment-a solar neutrino experiment in Russia; also, a spacecraft instrument for measuring atmospheric ozone profiles

SAMPEX Solar. Anomalous, and Magnetospheric Particles Explorer-NASA's first Small Explorer. launched in 1992

SAO Semiannual oscillation

SMM Solar Maximum Mission-a NASA solar observation satellite, launched in 1980

SOHO Solar and Heliospheric Observatory-ESA-NASA contribution to ISTP, scheduled for launch in 1995

SOUP Solar Optical Universal Polarimeter-Spacelab-2 experiment

Spacelab-2 Shuttle-borne solar physics/astrophysics mission conducted in 1985

Spartan 201 A retrievable shuttle-borne spacecraft to study the solar corona-flights conducted in 1993, 1994, 1995

STEP Solar-Terrestrial Energy Program

SUNRISE Sun's Radiative Input from Sun to Earth program to study the terrestrial consequences of solar variability

TIROS Television Infrared Observation Satellite - a family of polar-orhiting operational weather satellites

TOMS Total Ozone Mapping Spectrometer-the first of these instruments operated on NASA's Nimbus 7 satellite from 1978 to 1993, the second operated on Russia's Meteor-3 from 1991 to 1994, and additional examples will be flown on Russian, Japanese, and U.S. spacecraft between 1995 and 2000

UARS

Ulysses

Viking

Voyager

Wind

Yohkoh
Upper Atmosphere Research Satellite, launched from the shuttle in 1991

ESA-NASA mission, launched in 1990, to study the Sun's polar regions

Swedish satellite, launched in 1986 in near-polar orbit, to study particles and fields and obtain auroral images; also, a pair of NASA Mars orbiters/landers launched in 1975

A pair of NASA missions, launched in 1977, to explore the outer planets and heliosphere

NASA contribution to ISTP to study plasma phenomena upstream of Earth, launched in 1994

Japanese-U.S.-U.K. solar X-ray astronomy satellite, launched in 1991 
